\title{
Methods for flutter stability analysis of long-span bridges: a review
}

1 Tajammal Abbas $\mathrm{PhD}$

Postdoctoral Researcher, Chair of Modelling and Simulation of Structures, Bauhaus University Weimar, Weimar, Germany (corresponding author: tajammal.abbas@uni-weimar.de)

2 Igor Kavrakov MSc

Research Assistant, Research Training Group, Bauhaus University Weimar, Weimar, Germany

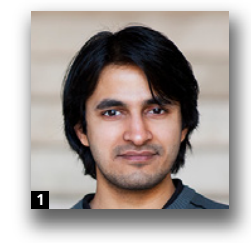

3 Guido Morgenthal PhD

Professor, Chair of Modelling and Simulation of Structures, Bauhaus University Weimar, Weimar, Germany

Predicting the phenomenon of aerodynamic instability is essential for the analysis and design of long-span cable-supported bridges. This paper reviews the history and development of aerodynamic analysis techniques and state-of-the-art numerical and experimental methods for flutter stability analysis. A discussion of the flutter phenomenon is followed by a literature review. This study provides a perspective on self-excited aerodynamic force models, identification of aerodynamic derivatives and methods for determining aerodynamic instability of long-span bridges in two dimensions and three dimensions. Computational fluid dynamics (CFD) techniques for modelling the flow past bluff bodies are also covered. Different model combination techniques are presented, utilising analytical, numerical and experimental approaches to predict the flutter limit. The effect of different parameters on the flutter stability limit is also highlighted. Furthermore, an overview of the complementary relationship between wind tunnel testing and CFD is provided. Finally, this paper also describes the engineering solutions adopted as countermeasures to prevent flutter instabilities.

Notation
$B$
$b$
$C$
$\mathbf{C}_{\mathrm{ae}}, \mathbf{K}_{\mathrm{ae}}$
$C_{\mathrm{D}}, C_{\mathrm{L}}, C_{\mathrm{M}}$
$C_{\mathrm{D}}^{\prime}, C_{\mathrm{L}}^{\prime}, C_{\mathrm{M}}^{\prime}$
$F$
$F_{\mathrm{D}}, F_{\mathrm{L}}, F_{\mathrm{M}}$
$f$
$G$
$H_{i}^{*}, A_{i}^{*}, P_{i}^{*}$
$h, \hat{h}, \bar{h}$
$h_{\mathrm{o}}, \alpha_{\mathrm{o}}$

chord

\section{half chord}

Theodorsen's circulation function aerodynamic damping and aerodynamic stiffness

static wind coefficient for drag, lift and moment

derivative of static wind coefficient for drag, lift and moment with respect to wind angle of attack

real part of Theodorsen's circulation function drag force, lift, force and moment oscillation frequency imaginary part of Theodorsen's circulation function

Scanlan's derivatives (with $i=1,6$ )

vertical displacement, velocity and acceleration vertical displacement and rotational displacement for forced oscillation simulations

$\begin{array}{ll}I_{Q, \alpha}, I_{Q, h}, I_{Q, p} & \begin{array}{l}\text { aerodynamic impulse response functions } \\ \text { (with } Q=\mathrm{L}, \mathrm{M}, \mathrm{D} \text { ) }\end{array} \\ K_{\alpha} & \begin{array}{l}\text { torsional stiffness of the deck } \\ \text { reduced frequency }\end{array} \\ k, K & \text { Küssner coefficients (with } i=1,2 \text { ) } \\ k_{i}^{\mathrm{a}}, k_{i}^{\mathrm{b}}, m_{i}^{\mathrm{a}}, m_{i}^{\mathrm{a}} & \text { modal mass, modal damping and modal } \\ \mathbf{M}, \mathbf{C}, \mathbf{K} & \text { stiffness } \\ m, I & \text { mass and mass moment of inertia } \\ p & \text { lateral displacement } \\ r & \text { radius of gyration } \\ \mathrm{St} & \text { Strouhal number } \\ T & \text { period of oscillation } \\ U_{\mathrm{cr}} & \text { flutter limit } \\ U_{\mathrm{d}} & \text { divergence velocity } \\ U_{\mathrm{r}} & \text { relative velocity } \\ U_{\infty} & \text { oncoming wind velocity } \\ v_{\mathrm{r}} & \text { reduced speed } \\ \ddot{x}, \dot{x}, x & \text { acceleration, velocity and displacement } \\ \alpha, \dot{\alpha}, \ddot{\alpha} & \text { rotational displacement, velocity and } \\ \alpha_{\mathrm{e}} & \text { acceleration } \\ & \text { effective angle of attack }\end{array}$




$\begin{array}{ll}\beta_{\mathrm{f}} & \text { ratio of measured flutter limit to flat plate } \\ \Delta t & \text { prediction } \\ \eta & \text { time step } \\ \mu & \text { empirical form factor } \\ & \text { ratio of the mass of the air moved by the } \\ & \text { deck to the mass of the deck } \\ \theta & \text { wind angles of attack } \\ v & \text { kinematic viscosity } \\ \xi_{h}, \xi_{\alpha} & \text { damping ratio for heave and pitch } \\ \rho & \text { mass density of the air } \\ \tau & \text { reduced time } \\ \Phi_{Q, \alpha}, \Phi_{Q, h}, \Phi_{Q, p} & \begin{array}{l}\text { aerodynamic indicial functions (with } Q=\mathrm{L} \\ \text { M, D) }\end{array} \\ \phi & \text { Wagner indicial function } \\ \omega & \text { natural circular frequency of oscillation } \\ \omega_{\mathrm{cr}} & \text { flutter frequency } \\ \omega_{h}, \omega_{\alpha} & \text { natural circular frequency for heave and pitch }\end{array}$

\section{Introduction}

The aeroelastic behaviour of long-span cable-supported bridges is a very important aspect to study as part of the analysis and design. Their increased flexibility makes these bridges highly sensitive to wind action, hence aerodynamic performance often becomes a decisive factor in the design process. Several aspects are highlighted in this paper including a brief history of long-span bridges, a description of aeroelastic instability phenomena, two-dimensional (2D) and threedimensional (3D) flutter modelling and analysis approaches and possible improvements in the structural system against flutter instability.

\subsection{History and development of long-span bridges}

Cable-supported bridges such as suspension and cable-stayed bridges are common to connect over great distances. The history of cable-supported bridges spans over two centuries. The first steel suspension bridge over the Jacob's Creek with $21 \mathrm{~m}$ span was built in 1801 (Jurado et al., 2011), whereas the first cable-supported bridge composed of drawn iron wires was built in Geneva in 1823. Gimsing and Georgakis (2011), Myerscough (2013) and Haifan (2011) describe the history and development of long-span cable-supported bridges around the world from the early nineteenth century.

The failure of the first Dryburgh Abbey Bridge in Scotland in 1818 due to wind is the first significant example where several of the stay connections failed during a storm. The Brighton Chain Pier, England (1836), the Tay Bridge, Scotland (1879) and the original Tacoma Narrows Bridge, USA (1940) are the most notable examples of long-span bridge failure where wind played a major role (Holmes, 2015). Gimsing and Georgakis (2011) and $\mathrm{Xu}$ (2013) summarise several examples of wind-induced bridge failure in the past two centuries.
Although some bridges failed prior to the collapse of the original Tacoma Narrows Bridge due to wind actions, this was the failure that decided bridge engineers to conduct scientific investigations of bridge aerodynamics. It was the third longest suspension bridge in the world with an $853 \mathrm{~m}$ main span. The deck started to oscillate with very large amplitudes in the vertical vibration modes at a wind speed of about $68 \mathrm{~km} / \mathrm{h}$ and around $45 \mathrm{~min}$ later the motion changed to a torsional mode (Chen and Duan, 1999). This eventually led to failure of some overstressed members and the main span collapsed as shown in Figure 1. The mechanics behind dynamic wind-structure interaction was not fully understood at that time. Traditional practices of design were based on experience alone and a lack of understanding of the nature of wind mainly contributed to this (Gimsing and Georgakis, 2011). Billah and Scanlan (1991) explained the mechanism of the ultimate failure of the bridge and demonstrated physically, as well as mathematically, that the main cause was not the forced resonance but the selfexcitation, which is aerodynamically induced self-excitation. Scanlan (2002) and Miyata (2003) summarise a brief history of some developments in the field of bridge aerodynamics after the collapse of Tacoma Narrows Bridge and explain how the investigations have helped in the development of experimental and analytical methods for the prediction of long-span bridge response to the wind.

Today, the spans of long-span suspension and cable-stayed bridges have been extended to new limits. The longest bridge in the world built at the end of the last century is the suspension bridge across the Akashi-Kaikyo Straits in Japan, with a main span of $1991 \mathrm{~m}$ and nearly $4 \mathrm{~km}$ of overall length.

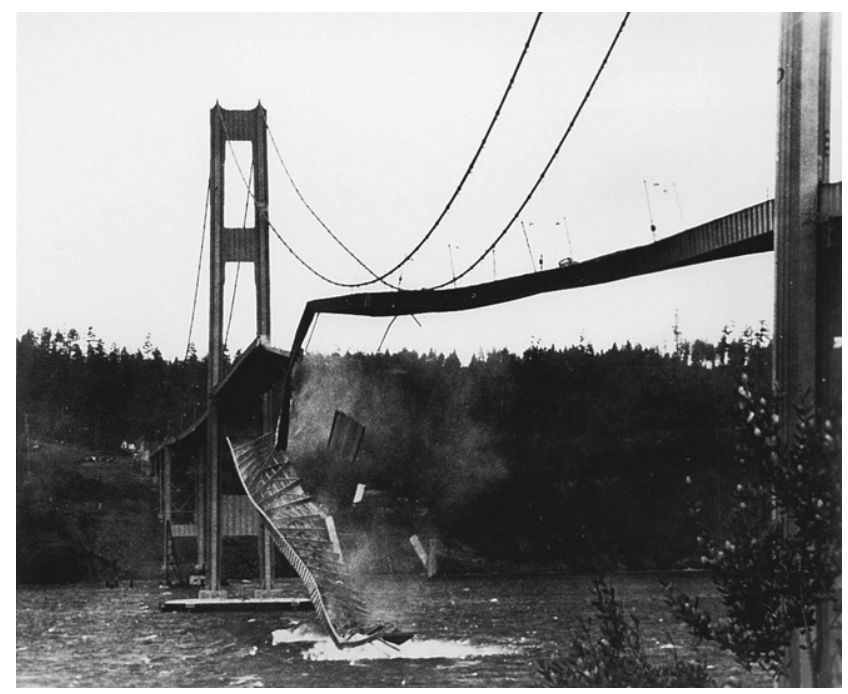

Figure 1. The original Tacoma Narrows Bridge collapse, USA (source: University Libraries (2017)) 
Aerodynamic characteristics governed the design of this bridge (Holmes, 2015). The Russky Bridge in Russia (2012) is the world's longest cable-stayed bridge, with a $1104 \mathrm{~m}$ long central span. $\mathrm{Xu}$ (2013) lists the top ten longest suspension bridges and the top ten longest cable-stayed bridges in the world. With the rapid growth of China's economy in the past couple of decades, more than 200 long-span bridges have been built. Ge and Xiang (2008a) provide the history and an account of new development of building bridges in China, with important structural properties and configurations of some bridges. Some examples of the major long-span bridges are listed in the Table 1 with their structural properties.

With the increase of bridge span length, modern bridge structures are more flexible, lightweight and their structural characteristics require special treatment of aerodynamic analysis and design under wind action. Weight (2009) provides an informative and critical analysis of the Great Belt East Bridge in Denmark, which is the third largest suspension bridge in the world. The proposed Messina Bridge takes wind engineering to its new limits, with a main span of $3300 \mathrm{~m}$. There has been an extensive study made on the deck configuration of the bridge. Bartoli et al. (2008) summarise wind tunnel tests, resulting in static wind coefficients, dynamic tests and numerical model time history analyses. An attempt has been made by Ge and Xiang (2007) to introduce a limit span length for suspension bridges and they later made an aerodynamic feasibility study of a $5000 \mathrm{~m}$ span suspension bridge along with aerodynamic stabilisation.

\subsection{Aeoelastic instability phenomena}

Dynamically flexible wind-sensitive structures, such as longspan bridges, oscillate under wind action. The motion of the structure generates aerodynamic forces which are known as motion-induced forces or self-excited forces. The overall system damping, which is the combination of structural damping and aerodynamic damping, becomes negative at very high wind speeds. If the effective damping is not sufficient to dissipate the energy input by the wind, the amplitudes of the oscillating structure start to grow and diverge. The theoretical border between the decaying and diverging motions is known as the critical condition and the corresponding wind velocity is called the critical wind velocity. The aerodynamic damping remains positive below the instability limit, which helps in reducing vibrations. The motion-induced part is insignificant for shortspan bridges and therefore there is no concern about aerodynamic instability Fujino and Siringoringo (2013b). The typical response amplitude of bridge elements due to increasing wind speed is shown in Figure 2.

Table 1. Some well-known long-span cable-supported bridges with their structural properties (Bartoli and Mannini, 2008; Ge and Xiang, 2008b; Haifan, 2011; Kvamstad, 2011; Larsen, 1998, 2006; Morgenthal et al., 2005; Ostenfeld et al., 1970; Selvam and Govindaswamy, 2001)

\begin{tabular}{|c|c|c|c|c|c|c|c|c|}
\hline Bridge name & Country & $\begin{array}{l}\text { Year } \\
\text { built }\end{array}$ & $\begin{array}{c}\text { Main } \\
\text { span: } m\end{array}$ & $\begin{array}{c}\text { Deck } \\
\text { width: } \mathrm{m}\end{array}$ & $\begin{array}{c}\text { Mass: } \\
10^{3} \mathrm{~kg} / \mathrm{m}\end{array}$ & $\begin{array}{c}\text { Mass moment } \\
\text { of inertia: } \\
10^{3} \mathrm{kgm}^{2} / \mathrm{m}\end{array}$ & $\begin{array}{c}\text { Bending } \\
\text { frequency: } \mathrm{Hz}\end{array}$ & $\begin{array}{c}\text { Torsional } \\
\text { frequency: } \mathrm{Hz}\end{array}$ \\
\hline \multicolumn{9}{|l|}{ Suspension bridge } \\
\hline Messina & Italy & Planned & 3300 & $60 \cdot 4$ & $55 \cdot 00$ & 28000 & 0.061 & 0.080 \\
\hline Akashi-Kaikyo & Japan & 1998 & 1991 & $35 \cdot 5$ & $43 \cdot 79$ & 9826 & 0.064 & $0 \cdot 150$ \\
\hline Zhejiang Xihoumen & China & 2008 & 1650 & $36 \cdot 3$ & - & - & $0 \cdot 100$ & 0.232 \\
\hline Great Belt (Storebælt) & Denmark & 1998 & 1624 & $31 \cdot 0$ & $22 \cdot 70$ & 2470 & 0.099 & $0 \cdot 272$ \\
\hline Little Belt (Lillebælt) & Denmark & 1970 & 600 & $33 \cdot 0$ & $11 \cdot 66$ & 1018 & 0.156 & 0.500 \\
\hline Tacoma Narrows & USA & 1940 & 854 & $12 \cdot 0$ & $4 \cdot 25$ & 178 & 0.130 & 0.200 \\
\hline Hålogaland & Norway & 2017 & 1145 & $18 \cdot 6$ & 11.94 & 355 & $0 \cdot 143$ & 0.441 \\
\hline Bosporus & Turkey & 1973 & 1074 & $28 \cdot 0$ & $13 \cdot 55$ & 1352 & $0 \cdot 162$ & 0.371 \\
\hline Runyang & China & 2005 & 1490 & - & - & - & $0 \cdot 124$ & 0.225 \\
\hline Tsing Ma & Hong Kong & 1997 & 1377 & - & - & - & - & - \\
\hline Humen & China & 1997 & 888 & - & - & - & $0 \cdot 112$ & 0.426 \\
\hline \multicolumn{9}{|l|}{ Cable-stayed bridge } \\
\hline Russky & Russia & 2012 & 1104 & - & - & - & - & - \\
\hline Sutong & China & 2008 & 1088 & $41 \cdot 0$ & - & - & $0 \cdot 171$ & 0.521 \\
\hline Stonecutters & Hong Kong & 2009 & 1018 & $53 \cdot 3$ & $32 \cdot 60$ & 297 & $0 \cdot 172$ & 0.324 \\
\hline Indiano & Italy & 1978 & 189 & $22 \cdot 4$ & $17 \cdot 40$ & 545 & 0.573 & $1 \cdot 179$ \\
\hline Guama & Brazil & - & 320 & $14 \cdot 2$ & $22 \cdot 51$ & 567 & 0.331 & 0.649 \\
\hline Tsurumi & Japan & 1994 & 510 & $38 \cdot 0$ & $32 \cdot 22$ & 2880 & $0 \cdot 204$ & 0.486 \\
\hline Normandy & France & 1995 & 856 & $23 \cdot 8$ & $13 \cdot 70$ & 633 & 0.222 & 0.500 \\
\hline \multicolumn{9}{|l|}{ Footbridge } \\
\hline Siena & Italy & 2006 & 59 & $3 \cdot 3$ & $1 \cdot 42$ & 0.810 & 1.735 & $4 \cdot 839$ \\
\hline Turin & Italy & 2003 & 156 & $6 \cdot 9$ & $3 \cdot 35$ & $13 \cdot 502$ & 0.510 & 0.590 \\
\hline Kehl-Straßburg & Germany-France & - & 183 & $6 \cdot 6$ & - & - & - & 0.650 \\
\hline
\end{tabular}


The static as well as dynamic structural response will, in general, increase with increasing mean wind speed (Strømmen, 2006). However, in the case of aeroelastic instability, the response is rapidly increasing for even a small increase in the mean wind speed. Therefore, the aerodynamic phenomena for long-span bridges can be mainly classified into two types based on the nature of amplitudes under wind action: limitedamplitude and divergent-amplitude wind-induced vibrations (Simiu and Scanlan, 1996). The former consist of buffeting, vortex-induced vibrations (VIV), rain-wind-induced vibrations and wake-induced vibrations, which are related to serviceable discomfort, increased internal stresses and may cause fatigue in the bridge structure. The latter comprise galloping, torsional flutter and coupled flutter, which can lead to structural instability. Instabilities resulting from wind-structure interaction are known as aerodynamic or aeroelastic instabilities. The term 'aeroelastic' emphasises the behaviour of deformed bodies, whereas 'aerodynamic' emphasises the rigid bodies (Chen and Duan, 1999). Sometimes these two terms are used

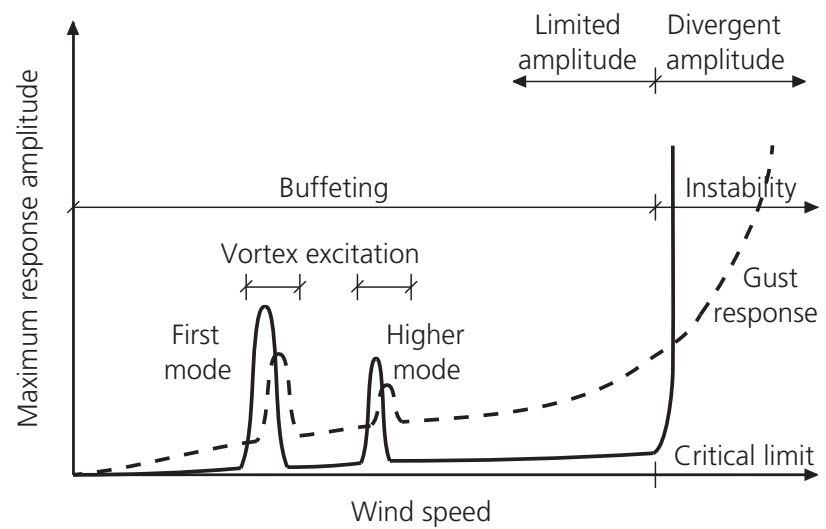

Figure 2. Schematic diagram of structural response plotted against wind speed (-, uniform flow; - -, turbulent flow) interchangeably. The oscillation amplitudes of the bridge deck can build up until this results in the failure of the bridge. The collapse of the original Tacoma Narrows Bridge is an example which resulted from just such aeroelastic instability.

\subsubsection{Classical flutter}

Flutter is a self-feeding aeroelastic phenomenon which is potentially destructive to long-span cable-supported bridges. The aerodynamic forces generated on the bridge deck couple with its motion and, if the energy input by the aerodynamic forces at high wind speeds in an oscillation cycle becomes larger than that dissipated by the mechanical damping of the bridge structural system, the amplitude of vibration will grow. This increasing vibration will then amplify the aerodynamic forces, resulting in continuously growing self-excited forces and self-exciting oscillations. If this situation continues for some time, it can lead to the ultimate limit state of the bridge and ultimately to collapse.

The term classical flutter was originally applied to thin aerofoils in the aircraft industry (Xu, 2013). Classical flutter is an aeroelastic phenomenon in which two degrees of freedom (2DOF) of a structure - torsional motion and vertical bending - couple together in a flow-driven, unstable oscillation (Simiu and Scanlan, 1996). It is also known as 2DOF flutter, coupled flutter or simply flutter. The critical flutter condition occurs at the wind speed where energy input to the system is equal to the energy dissipated by the structural damping. The oscillatory motions of the contributing degree of freedom (DOF) in the structure couple to create a single frequency called the flutter frequency, as shown in Figure 3. Flutter can happen in both laminar as well as in turbulent flows. When flutter occurs, the displacements become infinite according to the linear theory. Vertical and torsional motions occur with a phase difference, which is essential for the energy transfer from the wind to the structure. The mechanism of energy transfer in the case of coupled flutter is described in Figure 4. The flutter

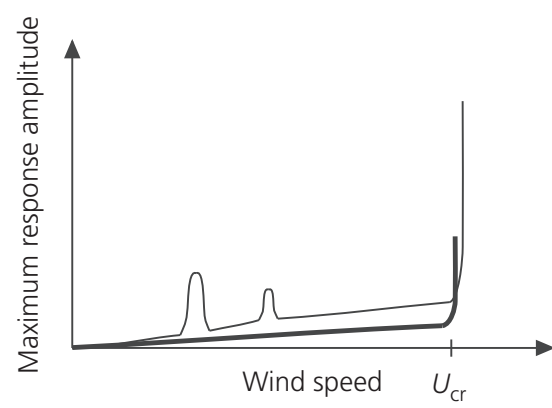

(a)

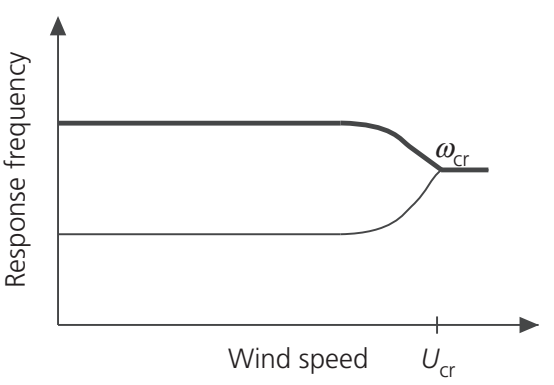

(b)

Figure 3. Schematic diagram of structural behaviour plotted against wind speed: (a) maximum response amplitudes; (b) response frequencies (-, heave mode; - , pitch mode; $U_{c r}$, flutter limit; $\omega_{c r}$, flutter frequency) 


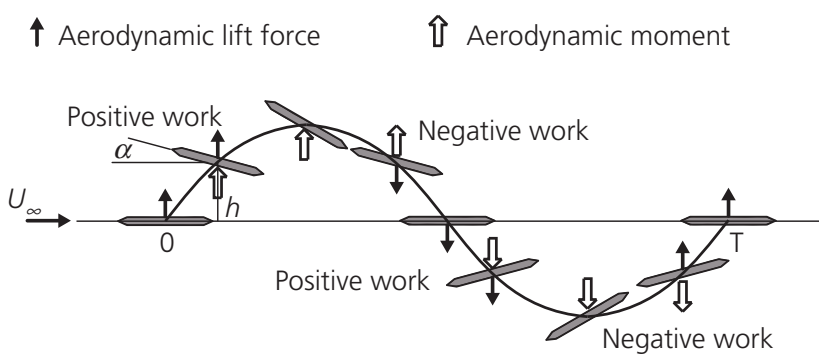

(a)

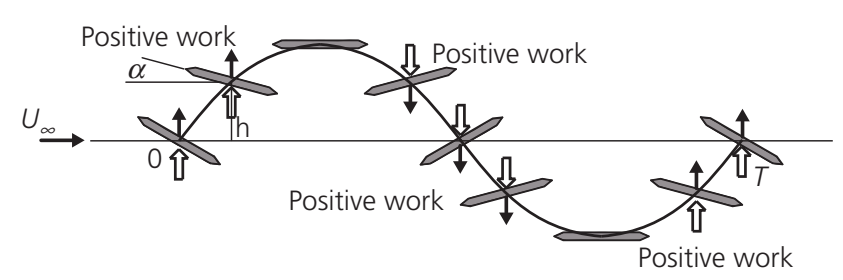

(b)

Figure 4. Excitation mechanism and energy effects of aerodynamic forces with: (a) $0^{\circ}$ phase difference; (b) $90^{\circ}$ phase difference $\left(U_{\infty}\right.$, wind speed; $h$, vertical displacement; $\alpha$, rotation; $T$, period of oscillation)

phenomena will depend on phase lag or degree of coupling among the modes (Fujino and Siringoringo, 2013b). A smaller frequency separation between the coupled modes increases the risk that flutter will occur (Dyrbye and Hansen, 1997). Selfexcited motion can even cause flutter on several DOF where higher modes also participate in the phenomenon.

The damping characteristics categorise the flutter behaviour of the system into a soft or a hard flutter. Chen and Kareem (2008) studied the rate of change in modal damping with increasing wind speed, which is referred to as soft or hard flutter. Hard-type flutter occurs by the rapid growth of negative aerodynamic damping with increasing wind speed beyond the flutter limit, whereas soft-type flutter happens where the negative damping builds up slowly with increasing wind speed.

Flutter is destructive in nature with infinitely large amplitudes according to the linear aerodynamic theory. In reality, aerodynamic non-linear effects take place which lead to limit cycle oscillation (LCO) where the amplitudes are limited. This difference can be visualised in Figure 5.

\subsubsection{Galloping}

Galloping is a single-degree-of-freedom (SDOF) large-amplitude aeroelastic oscillation in the transverse direction to wind. It occurs as a result of negative aerodynamic damping in the cross-wind direction. The amplitudes of oscillation can reach up to many times the section depth. It is sometimes also called

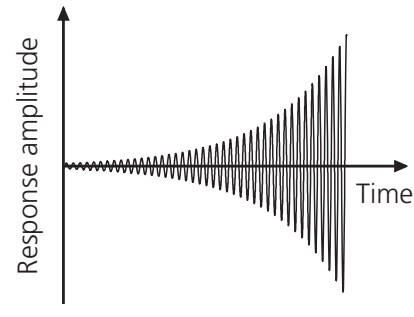

(a)

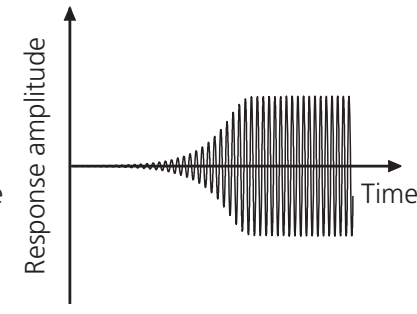

(b)
Figure 5. Schematic diagram of (a) flutter instability and (b) limit cycle oscillation

translational galloping, cross-wind galloping or bending flutter. Galloping is usually experienced by D-shaped crosssection, square section (at zero angle of attack) and circular section with some accretion, such as ice-coated transmission lines or guyed cables. It can also happen for a bridge with a bluff cross-section and a lightweight superstructure, even for a shorter span. The cross-section with larger aspect ratio (depth/width) has a higher tendency to gallop, which is known as soft galloping, whereas deeper sections require initial perturbation that leads to hard galloping.

Galloping occurs due to a change in the effective angle of attack of the vertical or the torsional motion of the structure. It can be evaluated by checking signs of the time-averaged static wind coefficients for lift and moment at zero angle of attack. A negative slope of static lift (or moment) coefficient usually indicates a tendency for galloping. The negative lift coefficient implies that the section will be pushed upward, resulting in a divergent response or galloping (Chen and Duan, 1999). Galloping depends mainly on the quasi-steady behaviour of the structure and therefore the mechanism of galloping can be explained by the quasi-steady aerodynamic theory. The analytical solution for galloping can be obtained from Dyrbye and Hansen (1997). The difference between the critical velocity predicted for galloping by linear and non-linear aerodynamic theory is insignificant (Fujino and Siringoringo, 2013b); however, the non-linear aerodynamic theory also provides amplitude and frequency. Wind tunnel experiments have been used in some studies to explain different aspects related to understanding the mechanism of galloping for rectangular cylinders with different aspect ratios (Washizu et al., 1978) and the galloping behaviour of Yadagawa Bridge in Japan (Ge et al., 2002).

\subsubsection{Torsional flutter}

Torsional flutter is a rotational SDOF aeroelastic instability. It is sometimes also referred to as rotational galloping, torsional galloping or stall flutter in aeronautical terminology. The mechanism of torsional flutter is related to the aerodynamic damping of rotational motion. Torsional flutter occurs when 
the total damping (mechanical plus aerodynamic) of the system in torsional motion becomes zero. The vertical mode of vibration of a structure does contribute somewhat, but the torsional action is more critical by far (Fujino and Siringoringo, 2013b). This occurs in long-span bridges with bluff deck sections, such as $\mathrm{H}$-shape, a rectangular section with larger aspect ratio, or truss sections, whereas streamlined sections tend to favour classical flutter.

Blevins and Iwan (1974) studied the mechanism of torsional flutter by linear and non-linear forms of quasi-steady theory. Nakamura and Mizota (1975) and Nakamura (1979) explained torsional flutter by theoretical and experimental study as an unsteady phenomenon. Nakamura (1979) also described torsional flutter by performing experimental forced vibration tests and provided the unsteady aerodynamic lifts and moments. Moreover, Washizu et al. (1980) studied rectangular cylinders with wind tunnel experiments and highlighted the effect of aspect ratio on the aeroelastic instability phenomena in the vicinity of the critical speed. Aerodynamic force with the same frequency as the Strouhal number, St, has a negative effect and can excite torsional motion in the deck (Zhou et al., 2006). Matsumoto et al. (1997, 2008d) and Matsumoto (2009) described the torsional flutter mechanism of rectangular and $\mathrm{H}$ shaped cylinders (Tacoma Narrows Bridge deck) and investigated the role of vortex excitation and Karman vortex excitation on the torsional flutter behaviour. Equations to solve the torsional flutter problem can be found in Dyrbye and Hansen (1997). In addition to these, the phenomenon of torsional flutter has been studied by several experimental (Daito et al., 2002) as well as numerical (Morgenthal et al., 2005) approaches.

\subsubsection{Aerostatic divergence}

Static divergence, sometimes also referred to as torsional divergence, is a phenomenon where torsional stiffness is lost at relatively high wind speed. It presents a static type of instability phenomenon where the structure does not oscillate. The divergence problem involves a combination of torsional divergence and lateral buckling. At a critical wind speed for torsional divergence, the bridge deck may experience out-of-plane buckling under the action of a drag force or torsionally diverge under a windinduced moment that increases with the increase in geometric twist angle (Chen and Duan, 1999). Matsumoto et al. (2010), additionally, classified torsional divergence into static SDOF torsional divergence and dynamic $2 \mathrm{DOF}$ torsional divergence.

\section{Motion-induced aerodynamic forces}

\subsection{Analytical models for flat plate}

Studies of the flutter phenomenon originated from the classical theory of aeroelasticity within aeronautical engineering. This discipline combines the theories of fluid dynamics and deformable solid mechanics (DSM) by imposing certain boundary conditions for the fluid-structure interaction (FSI). Historically, the earliest studies on this phenomenon are for aircraft wings in the beginning of the twentieth century. The description of the self-excited forces is based on a thin aerofoil, which can be described as a flat plate with half-chord $b$ with infinitesimal thickness, as depicted in Figure 6, forming the basis of the formulation for bluff bodies. All of the analytical flat plate models have a set of common assumptions: the fluid is inviscid and incompressible; the velocity field is irrotational, that is, they are based on the potential flow and small angles of attack; and the strip assumption applies with regard to the finite-span effect. This means that the aerodynamic force at any chord-wise section is the same as if that section was situated in a 2D flow. The study does not aim to cover all aspects of the development of flat plate aerodynamics, but rather gives a physical insight into the problem in order to be able to introduce semi-empirical models for bluff bodies. Dowell (2004), Fung (2002), Bisplinghoff et al. (1996) and Jurado and Hernández (2000) gave extensive attention to flat plate problems.

When subjecting a flat plate to a steady laminar flow with a direction rotated by an angle $\alpha$ with respect to the chord of the flat plate, a lift force $F_{\mathrm{L}}$ and a moment $F_{\mathrm{M}}$ are acting on the flat plate. These steady forces are a consequence of the distribution of the strength of vorticity around the body, the fluid density and the wind velocity. Considering a step rotation of the flat plate, a vortex is shed smoothly downstream from a single point of the trailing edge (also known as the Kutta-Joukowski condition). The circulation due to this free vortex has its counterpart on the flat plate of the same magnitude in order to satisfy the zero circulation condition, as assumed in the potential flow theory, which results in a force. The strength of the vorticity is time dependent, which results in an asymptomatic rise in the lift force towards steady-state forces. This was initially studied by Wagner (1925), who described the non-stationary lift force as a function of the reduced time by the so-called Wagner indicial function $\phi(\tau)$ in order to describe the circulatory part of the unsteady lift and moment (Fung, 2002)

$$
\text { 1a. } \quad F_{\mathrm{L}}=\rho \pi U_{\infty}^{2}\left(b \alpha^{\prime}+h^{\prime \prime}\right)+2 \pi \rho b U_{\infty}^{2}\left\{\int_{-\infty}^{\tau} \phi\left(\tau-\tau_{1}\right) \frac{\mathrm{d}}{\mathrm{d} \tau_{1}}\left[\alpha\left(\tau_{1}\right)+\frac{h^{\prime}\left(\tau_{1}\right)+0 \cdot 5 b \alpha^{\prime}\left(\tau_{1}\right)}{U_{\infty}} \frac{U_{\infty}}{b}\right] \mathrm{d} \tau_{1}\right\}
$$$$
\text { 1b. } \quad F_{\mathrm{M}}=-\rho b^{2} \pi U_{\infty}^{2}\left(\frac{1}{2} \alpha^{\prime}+\frac{1}{4} \alpha^{\prime \prime}\right)+\pi \rho b^{2} U_{\infty}^{2}\left\{\int_{-\infty}^{\tau} \phi\left(\tau-\tau_{1}\right) \frac{\mathrm{d}}{\mathrm{d} \tau_{1}}\left[\alpha\left(\tau_{1}\right)+\frac{h^{\prime}\left(\tau_{1}\right)+0 \cdot 5 b \alpha^{\prime}\left(\tau_{1}\right)}{U_{\infty}} \frac{U_{\infty}}{b}\right] \mathrm{d} \tau_{1}\right\}
$$ 


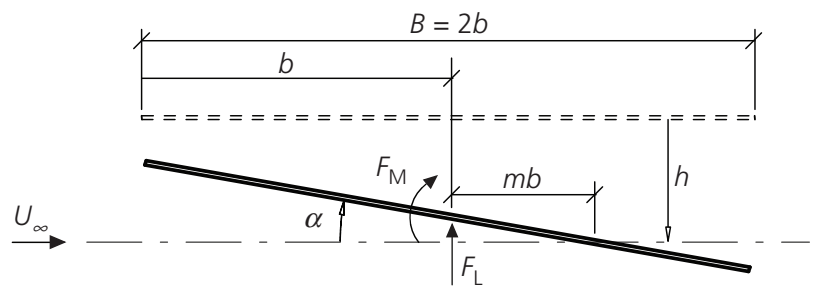

Figure 6. Definition of degrees of freedom (heave $h$ and pitch $\alpha$ ) and aerodynamic forces (lift $F_{\mathrm{L}}$ and moment $F_{\mathrm{M}}$ ) for a flat plate (- -, undeformed position; -, deformed position)

where $h$ is the vertical displacement, $\rho$ is the mass density of the air, $b$ is the half chord $(b=B / 2)$, and $U_{\infty}$ is the oncoming wind velocity. The reduced non-dimensional time is defined as $\tau=U_{\infty} t / b$ and the prime on $h$ and $\alpha$ is the differentiation with respect to the non-dimensional time. In addition to the circulatory forces, there are apparent mass forces due to the inertial effect related to the portion of fluid surrounding the body that moves with it, which are not associated with the creation of vorticity and are of minor importance in the bending-torsion flutter. In Figure 7(a), the approximation of the Wagner function by Jones (1940) is shown, from which it can be seen that, as $\tau \rightarrow \infty$, the indicial function $\phi \rightarrow 1$, which is the quasi-steady state. If the indicial function is considered as a step function, the quasisteady representation can be obtained (see Section 2.2.3). The quasi-steady model neglects the rise time of the indicial function, sometimes referred to as the fluid memory, by mapping the instantaneous state of the system at the current time into the corresponding state at an infinite time, such that the vortex has been moved far from the body for evaluating the flow-induced forces. The apparent mass forces are usually neglected when the quasi-steady assumption applies (Fung, 2002).

For a flat plate oscillating harmonically with a certain frequency, each of the vortices in the wake will influence the forcing on the flat plate; that is, the forcing will be dependent on the history of the motion or the so-called fluid memory effect. Theodorsen (1935) gave an exact solution of a harmonically oscillating flat plate for all reduced frequencies

2a. $\quad F_{\mathrm{L}}=\rho b^{2} \pi\left(U_{\infty} \dot{\alpha}+\ddot{h}\right)+2 \pi \rho b U_{\infty}^{2} C(k)\left(\alpha+\frac{\dot{h}+0 \cdot 5 b \dot{\alpha}}{U_{\infty}}\right)$

$2 b$.

$$
F_{\mathrm{M}}=-\rho b^{2} \pi\left(U_{\infty} \frac{b}{2} \dot{\alpha}+\frac{b^{2}}{8} \ddot{\alpha}\right)
$$$$
+\pi \rho b^{2} U_{\infty}^{2} C(k)\left(\alpha+\frac{\dot{h}+0 \cdot 5 b \dot{\alpha}}{U_{\infty}}\right)
$$

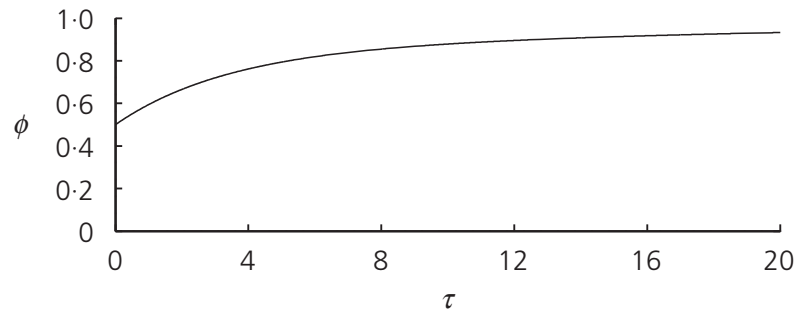

(a)

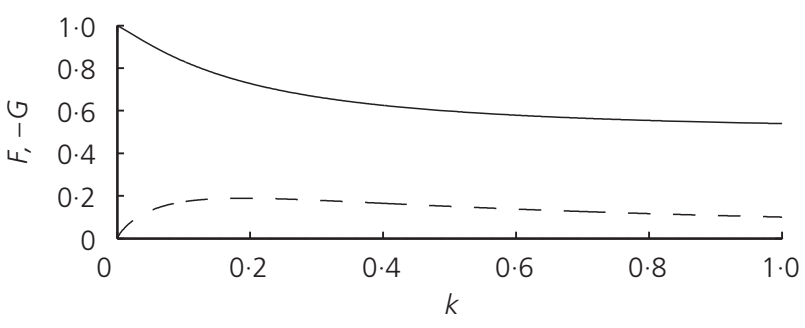

(b)

Figure 7. (a) Wagner function $\phi$ approximation by Jones with respect to reduced time $\tau=t U_{\infty} / b$. (b) Real $F(-)$ and imaginary $G(--)$ parts of Theodorsen's circulation function with respect to reduced frequency $k=\omega U_{\infty} / b$

where $C(k)=F(k)+i G(k)$ is the Theodorsen's circulation function, which depends on the reduced frequency $k=b \omega / U_{\infty}$ depicted in Figure 7(b). It describes the non-stationary contribution due to FSI. It can be seen for the low reduced frequencies $k \rightarrow 0$, the real part $F \rightarrow 1$ and the imaginary part $G \rightarrow 0$, which corresponds to the quasi-steady state. Garrick (1938) pointed out that Theodorsen's and Wagner's functions constitute a Fourier transform pair. In Peters (2008) an overview is given of the recent developments in unsteady aerofoil theory, including the closed-form solution and the solution using finite-state models (see Section 2.2.2).

\subsection{Semi-empirical models for bluff bodies}

There are four important actions to induce aerodynamic forces on bluff bodies, namely: static mean wind, vortex-induced excitation, the interaction between the wind and structural motion and, in the case of turbulent inflow conditions, additional aerodynamic force. These will correspondingly result in static mean wind force, vortex-excited force, motion-induced force and buffeting force, respectively (Ge and Xiang, 2008c), where the self-excited forces play the most important role in the bridge flutter phenomenon. The FSI in cases of general nonstreamlined bluff bodies is a complex phenomenon and to date, to the authors' knowledge, there is no general circulatory theory that can describe the motion-induced forces analytically, analogous to those for a flat plate based on the potential flow theory. This is a consequence of the flow separation and reattachment which could occur at multiple points on the solid 
boundary and it contradicts the assumption for the shedding of vortices from a single point. Therefore, a significant amount of research has been related to developing a semi-empirical model dependent on the motion and its time derivatives and additional empirically based aerodynamic derivatives. For a 2DOF section model, the system could be described (Tamura and Kareem, 2013) generally by

3a. $\quad m \ddot{h}+2 m \xi_{h} \omega_{h} \dot{h}+m \omega_{h}^{2} h=F_{\mathrm{L}}(h, \dot{h}, \ddot{h}, \alpha, \dot{\alpha}, \ddot{\alpha})$

3b. $\quad I \ddot{\alpha}+2 I \xi_{\alpha} \omega_{\alpha} \dot{\alpha}+I \omega_{\alpha}^{2} \alpha=F_{\mathrm{M}}(h, \dot{h}, \ddot{h}, \alpha, \dot{\alpha}, \ddot{\alpha})$

where $m$ and $I$ are the mass and mass moment of inertia, $\xi_{h}$ and $\xi_{\alpha}$ are the damping ratios and $\omega_{h}$ and $\omega_{\alpha}$ are the natural circular frequencies for the heave and pitch direction, $h$ and $\alpha$ are the vertical displacement and rotation, and $F_{\mathrm{L}}$ and $F_{\mathrm{M}}$ are the lift force and moment, respectively. In the case of bridge decks, the apparent mass terms dependent on the acceleration of the structures are usually neglected (Wu and Kareem, 2013). Considering a whole structure, the modal equation of motions are

4. $\mathbf{M} \ddot{x}(t)+\mathbf{C} \dot{x}(t)+\mathbf{K} x(t)=\mathbf{C}_{\mathrm{ae}} \dot{x}(t)+\mathbf{K}_{\mathrm{ae}} \dot{x}(t)$

where $\ddot{x}$ is the acceleration, $\dot{x}$ is the velocity and $x$ is the displacement. The left-hand side of Equation 4 represents the structural properties of the bridge deck, including modal mass $\mathbf{M}$, modal damping $\mathbf{C}$ and modal stiffness $\mathbf{K}$, whereas the right-hand side of the equation describes the unsteady motion-induced aerodynamic force, which can be divided into damping-related and stiffness-related terms. The term 'motion-induced' indicates that the force acting on the deck not only depends on the instantaneous value but also on the history of motion. Even if there is no coupling on the structural part, a coupling on the aerodynamic side could occur in the aerodynamic damping $\mathbf{C}_{\mathrm{ae}}$ and aerodynamic stiffness $\mathbf{K}_{\mathrm{ae}}$ matrices (Chen and Duan, 1999).

Historically, Duncan and Frazer (1928) first offered a 2D form for the flutter equations, followed by Bleich (1948) with a series of publications for truss bridges; however, the major milestone in bridge flutter was provided by Scanlan and Tomko (1971), who formulated the problem by expressing the motion-induced forces based on Theodorsen's theory and experimentally obtained aerodynamic derivatives, the so-called Scanlan derivatives, which are linearly dependent on the motion of the structure.

\subsubsection{Scanlan and equivalent representations}

The full expression of Scanlan's model is expressed as a linear function of the motion considered in Theodorsen theory multiplied by aerodynamic derivatives. Scanlan and Tomko (1971)

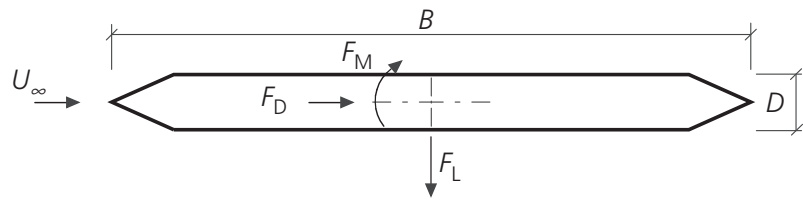

Figure 8. Definition of aerodynamic lift force $F_{\mathrm{L}}$, drag force $F_{\mathrm{D}}$ and moment $F_{M}$ on the section

introduced the self-excited forces as in Figure 8 by using aerodynamic derivatives obtained from an experimental approach

$5 a$.

$$
\begin{aligned}
F_{\mathrm{L}}= & \frac{1}{2} \rho U_{\infty}^{2} B\left(K H_{1}^{*} \frac{\dot{h}}{U_{\infty}}+K H_{2}^{*} \frac{B \dot{\alpha}}{U_{\infty}}+K^{2} H_{3}^{*} \alpha\right. \\
& \left.+K^{2} H_{4}^{*} \frac{h}{B}+K H_{5}^{*} \frac{\dot{p}}{U_{\infty}}+K^{2} H_{6}^{*} \frac{p}{B}\right)
\end{aligned}
$$

$5 b$.

$$
\begin{aligned}
F_{\mathrm{M}}= & \frac{1}{2} \rho U_{\infty}^{2} B^{2}\left(K A_{1} \frac{\dot{h}}{U_{\infty}}+K A_{2} \frac{B \dot{\alpha}}{U_{\infty}}+K^{2} A_{3} \alpha\right. \\
& \left.+K^{2} A_{4} \frac{h}{B}+K A_{5} \frac{\dot{p}}{U_{\infty}}+K A_{6} \frac{p}{B}\right)
\end{aligned}
$$

$5 c$

$$
\begin{aligned}
F_{\mathrm{D}}= & \frac{1}{2} \rho U_{\infty}^{2} B\left(K P_{1}^{*} \frac{\dot{p}}{U_{\infty}}+K P_{2}^{*} \frac{B \dot{\alpha}}{U_{\infty}}+K^{2} P_{3}^{*} \alpha\right. \\
& \left.+K^{2} P_{4}^{*} \frac{p}{B}+K P_{5}^{*} \frac{\dot{h}}{U_{\infty}}+K^{2} P_{6}^{*} \frac{h}{B}\right)
\end{aligned}
$$

where $F_{\mathrm{D}}$ is the drag force, $p$ is the lateral displacement, $K=B \omega / U_{\infty}$ is the reduced frequency, $\omega$ is the frequency of oscillation and $H_{i}^{*}, A_{i}^{*}$ and $P_{i}^{*}$ (with $i=1,6$ ) are Scanlan's derivatives. These aerodynamic derivatives for bluff sections cannot be computed analytically; however, it is possible to obtain them by experimental wind tunnel tests or through CFD simulations; this is also discussed in Section 2.5.1.

The motion-induced aerodynamic forces are based on two assumptions (Ge and Xiang, 2009b). First, the displacement of the structure follows harmonic vibration and can be represented by 18 aerodynamic derivatives neglecting the apparent mass for the acceleration term. Second, if the motion-induced forces are considered to have a non-linear relationship with structural motion, then these expressions would include the second-order or even high-order terms of the structural motion. Scanlan's model has been widely used for the solution of $2 \mathrm{D}$ and $3 \mathrm{D}$ aeroelastic instability problems in the field of bridge aerodynamics, owing to its ability to be applied to different types of bridge cross-sections. Scanlan and Tomko (1971) 
initially studied free vibration methods to identify aerodynamic derivatives $\left(H_{i}^{*}, A_{i}^{*}\right.$ with $\left.i=1,3\right)$ by using experiments for five different deck shapes and studied the effect of the change in depth of H-shaped section. The reason for using three aerodynamic derivatives was the direct relationship with the flat plate theory, which is based only on vertical motion, angular rotation and angular velocity in the circulatory terms. Scanlan (1978a, 1978b) utilised $\left(H_{i}^{*}, A_{i}^{*}, P_{i}^{*}\right.$ with $\left.i=1,3\right)$ aerodynamic derivatives for the linear dynamic analysis of bridge flutter for bridges having lateral vibration modes along with the energy considerations involved in the assessment of aerodynamic stability. Jain et al. (1996a, 1996b) made use of all six $P_{i}^{*}$ aerodynamic derivatives in their aerodynamic analysis.

In the field of bridge aerodynamics, comparing aerodynamic characteristics utilising the aerodynamic derivatives is a common and widely accepted approach. Various conventions have been developed and are used for this purpose based on the same assumptions as were used by Scanlan. The earlier ones were developed by Miyata et al. (1975). Zasso (1996) gave a representation of the motion-induced forces with the advantage of a better description in the low-frequency range where Scanlan's tend to become zero. The coefficients $\left(H_{i}^{*}, A_{i}^{*}\right)$ are the real and the imaginary parts of the transfer functions between the non-dimensional forces and the non-dimensional displacements. Motion-induced aerodynamic forces are mathematically described in real number or complex number expressions and flutter analyses performed using either of these lead to the same numerical results. Starossek (1992) represented the motion-induced forces in a complex form with the advantage in the solution method by solving a linear eigenvalue problem wherein the complex flutter mode shape is obtained without additional effort.

In the aerodynamic study for the Normandy Bridge in France, a so-called Küssner notation was used, where the aerodynamic derivatives are known as Küssner coefficients $\left(k_{i}^{\mathrm{a}}, k_{i}^{\mathrm{b}}, m_{i}^{\mathrm{a}}, m_{i}^{\mathrm{a}}\right.$ with $i=1,2)$ (Szechenyi, 1973). Zasso (1996) and Borri and Höffer (2000) summarised some of these conventions and highlighted their merits and disadvantages. Jensen (1997) compared Scanlan's derivatives with those developed for use in marine engineering, based on the relative position of the bridge deck, utilising the velocity and acceleration terms. Some of these conventions are summarised in Table 2.

\subsubsection{Impulse and indicial function representations}

The Scanlan's representation of motion-induced forces includes time- and frequency-dependent terms. In order to conduct a pure time-domain analysis, the aeroelastic forces should be formulated without the frequency-dependent terms. This is realised by indicial and impulse functions, which are sometimes referred to as approximation kernels. In fact, they are closely related to each other and, as pointed out in $\mathrm{Wu}$ and Kareem (2014b), the impulse function is the derivative of the indicial function with respect to time, with an exception at $t=0$. The formulation with respect to indicial function is obtained by expanding the Wagner formulation of non-stationary forces for a bluff body and neglecting the non-circularity terms related to apparent mass (Caracoglia and Jones, 2003b)

$6 a$.

$$
\begin{aligned}
F_{\mathrm{L}}= & -\frac{1}{2} \rho U_{\infty}^{2} B C_{\mathrm{L}}^{\prime} \int_{-\infty}^{\tau}\left[\Phi_{\mathrm{L}} \alpha\left(\tau-\tau_{1}\right) \alpha^{\prime}\left(\tau_{1}\right)\right. \\
& \left.+\Phi_{\mathrm{L}} h\left(\tau-\tau_{1}\right) \frac{2 h^{\prime \prime}\left(\tau_{1}\right)}{B}+\Phi_{\mathrm{L} p}\left(\tau-\tau_{1}\right) \frac{2 p^{\prime \prime}\left(\tau_{1}\right)}{B}\right] \mathrm{d} \tau_{1}
\end{aligned}
$$

$6 b$

$$
\begin{aligned}
F_{\mathrm{M}}= & \frac{1}{2} \rho U_{\infty}^{2} B^{2} C_{\mathrm{M}}^{\prime} \int_{-\infty}^{\tau}\left[\Phi_{\mathrm{M}} \alpha\left(\tau-\tau_{1}\right) \alpha^{\prime}\left(\tau_{1}\right)\right. \\
& \left.+\Phi_{\mathrm{M}} h\left(\tau-\tau_{1}\right) \frac{2 h^{\prime \prime}\left(\tau_{1}\right)}{B}+\Phi_{\mathrm{M} p}\left(\tau-\tau_{1}\right) \frac{2 p^{\prime \prime}\left(\tau_{1}\right)}{B}\right] \mathrm{d} \tau_{1}
\end{aligned}
$$

Table 2. Conversion between the notation for the aerodynamic derivatives by Scanlan, Starossek, Küssner, Zasso and Jensen

$\begin{array}{lllllllll}\text { Scanlan } & H_{4}^{*} & H_{1}^{*} & A_{4}^{*} & A_{1}^{*} & H_{3}^{*} & H_{2}^{*} & A_{3}^{*} & A_{2}^{*} \\ \text { Starossek } & \frac{\pi}{2} c_{h h}^{\prime} & \frac{\pi}{2} c_{h h}^{\prime \prime} & \frac{\pi}{4} c_{\alpha h}^{\prime} & \frac{\pi}{4} c_{\alpha h}^{\prime \prime} & \frac{\pi}{4} c_{h \alpha}^{\prime} & \frac{\pi}{4} c_{h \alpha}^{\prime \prime} & \frac{\pi}{8} c_{\alpha \alpha}^{\prime} & \frac{\pi}{8} c_{\alpha \alpha}^{\prime \prime} \\ \text { Küssner } & \frac{-v_{r}^{2}}{2 \pi} k_{1}^{a} & \frac{-v_{r}^{2}}{2 \pi} k_{2}^{a} & \frac{-v_{r}^{2}}{4 \pi} m_{1}^{a} & \frac{-v_{r}^{2}}{4 \pi} m_{2}^{a} & \frac{-v_{r}^{2}}{4 \pi} k_{1}^{b} & \frac{-v_{r}^{2}}{4 \pi} k_{2}^{b} & \frac{-v_{r}^{2}}{8 \pi} m_{1}^{b} & \frac{-v_{r}^{2}}{8 \pi} m_{2}^{b} \\ \text { Zasso } & \frac{\pi}{2} h_{4}^{*} & \frac{-v_{r}}{2 \pi} h_{1}^{*} & \frac{\pi}{2} a_{4}^{*} & \frac{-v_{r}}{2 \pi} a_{1}^{*} & \frac{v_{r}^{2}}{4 \pi^{2}} h_{3}^{*} & \frac{-v_{r}}{2 \pi} h_{2}^{*} & \frac{v_{r}^{2}}{4 \pi^{2}} a_{3}^{*} & \frac{-v_{r}}{2 \pi} a_{2}^{*} \\ \text { Jensen } & -Y_{r}^{\prime} & Y_{v}^{\prime} \frac{1}{K} & -N_{r}^{\prime} & N_{v}^{\prime} \frac{1}{K} & -\left(Y_{\dot{r}}^{\prime}+Y_{v}^{\prime} \frac{1}{K^{2}}\right) & \left(Y_{r}^{\prime}-Y_{\dot{v}}^{\prime}\right) \frac{1}{K} & -\left(N_{\dot{r}}^{\prime}+N_{v}^{\prime} \frac{1}{K^{2}}\right) & \left(N_{r}^{\prime}-N_{\dot{v}}^{\prime}\right) \frac{1}{K}\end{array}$


forces were initially introduced only for lift and moment by Scanlan et al. (1974) for bridge decks. Lin and Ariaratnam (1980) and Lin and Li (1993) simplified this formulation considering only rotational motion in order to study the torsional stability in turbulent wind. These were then extended by Scanlan (1993) for the drag force based on torsional and lateral motion and the final form was used after Jain et al. (1996b) studied the effect of the horizontal motion on the selfexcited forces.

The phenomenon of 'overshooting' occurs for bluff bodies in which the indicial function has a higher value than unity in the rise time and then drops. This is a consequence of the flow separation and it is addressed by Scanlan (2000) and $\mathrm{Wu}$ and Kareem (2014b). Comparing to the original flat plate theory, this form does not incorporate the indicial function dependent on the angular acceleration term $\alpha^{\prime \prime}$. These terms were originally included in the notation by Scanlan et al. (1974); however, Lin and Yang (1983) and Bucher and Lin (1988, 1989) described this as a redundant relation, given that the angular velocity $\alpha^{\prime}$ incorporates information about the angular acceleration $\alpha^{\prime \prime}$ in the interval $[-\infty ; \tau]$. Changing the order of integration by partial integration, the limits of the integral change from $\infty$ to 0 ; thus, numerical integration is in order. The impulse function formulation was initially given by Lin and Yang (1983) and then by Bucher and Lin (1988, 1989)

$7 a$.

$$
\begin{aligned}
F_{\mathrm{L}}= & -\frac{1}{2} \rho U_{\infty}^{2} \int_{-\infty}^{t}\left[I_{\mathrm{L} \alpha}\left(t-\tau_{1}\right) \alpha\left(\tau_{1}\right)\right. \\
& \left.+I_{\mathrm{L} h}\left(\tau-\tau_{1}\right) h\left(\tau_{1}\right)+I_{\mathrm{L} p}\left(\tau-\tau_{1}\right) p\left(\tau_{1}\right)\right] \mathrm{d} \tau_{1}
\end{aligned}
$$

$7 b$.

$$
\begin{aligned}
F_{\mathrm{M}}= & \frac{1}{2} \rho U_{\infty}^{2} \int_{-\infty}^{t}\left[I_{\mathrm{M} \alpha}\left(t-\tau_{1}\right) \alpha\left(\tau_{1}\right)\right. \\
& \left.+I_{\mathrm{M} h}\left(\tau-\tau_{1}\right) h\left(\tau_{1}\right)+I_{\mathrm{M} p}\left(\tau-\tau_{1}\right) p\left(\tau_{1}\right)\right] \mathrm{d} \tau_{1}
\end{aligned}
$$

7c.

$$
\begin{aligned}
F_{\mathrm{D}}= & \frac{1}{2} \rho U_{\infty}^{2} \int_{-\infty}^{t}\left[I_{\mathrm{D} \alpha}\left(t-\tau_{1}\right) \alpha\left(\tau_{1}\right)\right. \\
& \left.+I_{\mathrm{D} h}\left(\tau-\tau_{1}\right) h\left(\tau_{1}\right)+I_{\mathrm{D} p}\left(\tau-\tau_{1}\right) p\left(\tau_{1}\right)\right] \mathrm{d} \tau_{1}
\end{aligned}
$$

where $I_{Q, \alpha}, I_{Q, h}, I_{Q, p}$ (with $Q=\mathrm{L}, \mathrm{M}, \mathrm{D}$ ) are aerodynamic impulse response functions describing the lift, moment and drag force evolution for a certain time period due to change of the motion at a certain time instant $\alpha, h, p$, respectively. The determination of the impulse and indicial function is discussed in
Section 2.5.2. Wu and Kareem (2014b) compared both the indicial and impulse function approaches for bluff body aerodynamics on a theoretical, experimental and numerical basis, concluding that they are essentially very similar, if not the same.

\subsubsection{Quasi-steady representations}

The quasi-steady theory for bluff bodies essentially does not differ from the one for flat plates. This model does not consider the fluid memory effect; however, it offers a static nonlinear relationship between the instantaneous angle of attack and the flow-induced forces. Ignoring the apparent mass for laminar flow, the self-excited forces can be represented as (Borri and Costa, 2004; Kovacs et al., 1992)

8a. $\quad F_{\mathrm{L}}=-\frac{1}{2} \rho U_{\mathrm{r}}^{2} B C_{\mathrm{L}}\left(\alpha_{\mathrm{e}}\right)$

8b. $\quad F_{\mathrm{M}}=\frac{1}{2} \rho U_{\mathrm{r}}^{2} B^{2} C_{\mathrm{M}}\left(\alpha_{\mathrm{e}}\right)$

8c. $\quad F_{\mathrm{D}}=\frac{1}{2} \rho U_{\mathrm{r}}^{2} B C_{\mathrm{D}}\left(\alpha_{\mathrm{e}}\right)$

where

9a. $U_{\mathrm{r}}=\sqrt{\left(U_{\infty}-\dot{p}\right)^{2}+\left(\dot{h}+m_{i} B \dot{\alpha}\right)^{2}}$

9b. $\quad \alpha_{\mathrm{e}}=\alpha+\left(\frac{\left.\dot{h}+m_{i} B \dot{\alpha}\right)}{U_{\infty}-\dot{p}}\right)$

With the effective angle of attack $\alpha_{\mathrm{e}}$ and relative velocity $U_{\mathrm{r}}$, the fluid-structure interaction is described. The major challenge in this model is to determine the parameter $m_{i}$ for $i=\mathrm{L}, \mathrm{M}, \mathrm{D}$, which describes the distance between the aerodynamic and stiffness centres. This coefficient depends on the pressure distribution, as well as the separation of the fluid from the body and, in fact, is non-linear and frequency dependent; therefore, there are challenges in its determination. Diana et al. $(1993,2013)$ determined this coefficient based on the flutter derivatives at a certain reduced velocity, for which the quasi-steady assumption holds, as depicted in Table 3. Wu and Kareem (2013) included an

Table 3. Conversion between the notation for the aerodynamic derivatives by Scanlan and corrected quasi-steady theory

$\begin{array}{lllllllll}\text { Scanlan } & H_{1}^{*} & H_{2}^{*} & H_{3}^{*} & H_{4}^{*} & A_{1}^{*} & A_{2}^{*} & A_{3}^{*} & A_{4}^{*} \\ \text { Quasi-steady } & -\left(C_{\mathrm{L}}^{\prime}+C_{\mathrm{D}}\right) \frac{v_{\mathrm{r}}}{2 \pi} & -\left(C_{\mathrm{L}}^{\prime}+C_{\mathrm{D}}\right) m_{\mathrm{L}} \frac{v_{\mathrm{r}}}{2 \pi} & \mathrm{C}_{\mathrm{L}}^{\prime} \frac{v_{\mathrm{r}}^{2}}{(2 \pi)^{2}} & -2 C_{\mathrm{L}} \frac{v_{\mathrm{r}}}{2 \pi} & -C_{\mathrm{M}}^{\prime} \frac{v_{\mathrm{r}}}{2 \pi} & C^{\prime} M m_{\mathrm{M}} \frac{v_{\mathrm{r}}}{2 \pi} & C_{\mathrm{L}} \frac{v_{\mathrm{r}}^{2}}{(2 \pi)^{2}} & -2 C_{\mathrm{M}} \frac{v_{\mathrm{r}}}{2 \pi}\end{array}$


extended discussion on the importance of this parameter in determining equivalent steady state for applying the quasi-steady model. Linearising, taking quarter chord distance for $m$ $(m=0 \cdot 25)$, the quasi-steady model for a flat plate is obtained. In order to take into account the fluid memory term, Diana et al. (1992, 1993) introduced additional factors $K_{\mathrm{D}}, K_{\mathrm{L}}, K_{\mathrm{M}}$ for the static wind coefficients to develop the corrected quasi-steady theory

10a. $C_{\mathrm{D}}\left(\alpha_{\mathrm{e}}\right)=C_{\mathrm{D}}\left(\alpha_{0}\right)+\int_{\alpha_{0}}^{\alpha_{\mathrm{e}}} K_{\mathrm{D}} \mathrm{d} \alpha$

10b. $\quad C_{\mathrm{L}}\left(\alpha_{\mathrm{e}}\right)=C_{\mathrm{L}}\left(\alpha_{0}\right)+\int_{\alpha_{0}}^{\alpha_{\mathrm{e}}} K_{\mathrm{L}} \mathrm{d} \alpha$

10c. $\quad C_{\mathrm{M}}\left(\alpha_{\mathrm{e}}\right)=C_{\mathrm{M}}\left(\alpha_{0}\right)+\int_{\alpha_{0}}^{\alpha_{\mathrm{e}}} K_{\mathrm{M}} \mathrm{d} \alpha$

According to Diana et al. $(1993,2013)$ these three parameters can be obtained for high reduced velocities from the flutter derivatives or through wind-tunnel testing (Diana et al., 1992, 1995; Falco et al., 1992). Table 3 shows the relation between Scanlan's representation and quasi-steady theory for a selected reduced velocity. Another modified quasi-steady model was developed by Øiseth et al. (2010) for a case study on Norway's Hardanger Bridge. This model was based on the frequencyindependent interpolation of the flutter derivatives at the structural frequency and it seems to correspond well with the other models for the specific case study.

\subsubsection{Recent developments}

Recently, Diana et al. (2008) developed a new concept of modelling the aerodynamic forces based on the hysteresis behaviour of the force and moment coefficient with respect to the dynamic angle of attack for different reduced velocities. The challenge, in this case, is to formulate a rheological element which could encapsulate the unsymmetrical non-linear hysteresis. The model is based on several independent mechanical oscillators in a parallel configuration, whose parameters are obtained through the concept of an aerodynamic force transfer function. In Wu and Kareem (2011), a non-parametric model was developed based on an input-output black box, artificial neural network (ANN) framework presented in order to describe the hysteretic behaviour. Although promising results are shown for the coupled gust-induced and motioninduced forces, the model was still not applied for the computation of the flutter velocity. However, the same concept of ANN was used by Wu and Kareem (2013) for approximating directly the aerodynamic hysteresis with respect to the angle of incidence in the modified hybrid model, where the flutter velocity was obtained. To further investigate the aerodynamic non-linearities, Wu and Kareem (2014a) used the Volterra series as approximation kernels to simulate the non-linear system with fading memory term due to the viscosity. With this formulation, the non-linear system is modelled as an infinite sum of multidimensional convolution integrals of increasing order, which directly correlates to the unsteady aerodynamic forces. Comparison of the modelling schemes and discussions on the topic can be found in Scanlan (1993, 2000, 2001), Chen and Kareem (2002) and Wu and Kareem (2013).

\subsection{Numerical methods}

The aerodynamic behaviour for the design of long-span bridges is traditionally studied through wind tunnel tests; however, these tests are expensive in terms of cost and require time for planning and actual testing, so may be prohibitive at the design stage. Detailing can be easy, but a small modification of the global shape of the section requires a new model. CFD techniques have gained much attention and development in the last two decades and are used as an alternative alongside the analytical and experimental methods. The techniques are based on the numerical discretisation of the Navier-Stokes (N-S) equations and, by introducing a shape similar to a bridge deck immersed in the flow, it is possible to determine the force coefficients, pressure distribution, aerodynamic derivatives and flow visualisation, which leads to an insightful understanding of the aerodynamic phenomena. However, still, this has limitations with regard to the complexity and nature of 3D bridge structures (Larsen, 1997). CFD is more accessible than wind tunnel testing for the aerodynamic analysis of bridge deck geometry and for checking the performance of a structure, as pointed out by Ge and Xiang (2008a). Blocken (2014) provides a perspective on the past, present and future of CFD application in wind engineering or the so-called field of computational wind engineering (CWE).

There are various methods for the discretisation of the flow field; however, the most commonly used in the field of bridge aerodynamics are the finite-element method (FEM), finitevolume method (FVM) and discrete-vortex method (DVM). The FVM and FEM methods are Eulerian mesh-based methods with the main difference that the FEM also models the flow between nodal points utilising the shape functions, whereas the DVM is Lagrangian mesh-free. Earlier studies based on the FEM (Frandsen, 2004) did not include turbulence models for the closure of the $\mathrm{N}-\mathrm{S}$ equations and, although the computations yielded comparable results, a remark suggesting further elaborate studies was made. Most recent results based on the FEM and FVM include turbulence models such as the Reynolds-average Navier-Stokes (RANS) models based on time averaging of the flow, large eddy simulation (LES) based on spatial averaging of the flow and hybrid 
models such as detached eddy simulation (DES) and hybrid RANS-LES models. An early comparison was made by Bruno and Khris (2003) for 2D numerical models using the FVM with RANS and 2D LES as turbulence models, including parametric studies for the computational parameters, where emphasis was given on the importance of modelling of the deck equipment. Recently, Brusiani et al. (2013) made a comparison of the 2D two-equation RANS-based models ( $\kappa-\epsilon, \kappa-\omega$ and $\kappa-\omega$ shear-stress transport) for the determination of the flutter derivatives, concluding that all of them yielded comparable results. The differences between 2D and $3 \mathrm{D}$ simulations with respect to flutter derivatives were studied in Bai et al. (2010) and Ketong and Aiping (2014) using the DES turbulence model with FVM. The first reference commented regarding the importance of the $3 \mathrm{D}$ CFD computations for complex geometries; however, comparable results were obtained for a generic streamlined deck between the 2D and 3D simulations. The DVM gained much attention in the 1990s for use in the field of bridge aerodynamics. The success of the simulations is attributed to the bluff nature of the crosssections and to the 2D nature of flow around bridge girders (Walther, 1994). It offers significantly lower numerical diffusion compared to mesh methods and high computational efficiency; however, various a priori numerical parameters and an insufficient number of discrete vortices might be seen as a disadvantage, which results in a model rather than a direct numerical simulation. However, the latter could be reduced by introducing the vortex-in-cell technique, formulating a background grid for maintaining evenly spaced particles. It should be noted that this grid is for practically averaging the vorticity and it does not introduce any numerical diffusion (Morgenthal, 2002). It has been used to study flow around a flat plate, as well as other bluff bridge deck cross-sections, to show the applicability and efficiency of the method and to help understand mechanisms related to the phenomena of aeroelastic instability (Abbas, 2016; Abbas and Morgenthal, 2013a, 2013c, 2014a, 2014b; Larsen and Walther, 1997a; Morgenthal, 2005, 2006; Morgenthal and Walther, 2007; Morgenthal et al., 2014; Taylor and Vezza, 1999). The aim of the study is not to offer a critical in-depth comparison of the various CFD techniques, but rather to present them as an alternative method in the procedure for determining the critical flutter limit. Furthermore, a list of studies is presented in Tables 4 and 5; these are offered for further reference. An insightful comparison of the numerical methods is made by Ge and Xiang (2008c) where the three methods were compared for a flat plate and bluff bodies, all resulting in comparable results.

\subsection{Experimental methods}

\subsubsection{Full-scale monitoring}

Wind tunnel tests are the basis for the analysis and design of long-span bridges. However, small-scale experiments have limitations in predicting the full-scale behaviour owing to scale effects such as Reynolds number and structural damping. The modelling parameters can only be validated through full-scale measurements, which are crucial for bridge aerodynamics. Full-scale measurements are seldom performed for long-span bridges. Frandsen (2001) utilised full-scale measurements on the Great Belt Suspension Bridge and provided several other references of full-scale measurements on different bridges around the world. Moreover, full-scale measurements have been used to validate the analytical response prediction (Jones and Ozkan, 2002) and to extract aerodynamic derivatives (Nikitas et al., 2008). The full-scale aeroelastic behaviour of bridges is still not completely understood and is an active research topic.

\subsubsection{Wind tunnel testing}

Tests in wind tunnels are commonly carried out either on section models, taut-strip models or full aeroelastic models. These tests require scaled models representing the structure by ensuring certain similarity laws. Wind tunnel tests for the Akashi Kaikyo Bridge by Miyata and Yamaguchi (1993) and Great Belt East Bridge by Larsen (1993) are notable examples of 3D aeroelastic bridge models. Moreover, Sato et al. (2002) and Yoshizumi and Inoue (2002) describe 2D and 3D flutter analyses of full aeroelastic model tests for long-span bridges.

Section models are more commonly used for defining static wind loads (Davenport and King, 1984) and measuring aerodynamic derivatives of the section. These tests are also useful to study the aeroelastic instability characteristics of the deck by making use of the wind tunnel experiments on dynamically mounted section models. Matsumoto (1996) studied aerodynamic derivatives for prisms and their dependence on each other, and categorised low-speed torsional flutter, high-speed torsional flutter and coupled flutter from the unsteady pressure measurement under SDOF heaving/torsional forced vibration. Table 6 provides some examples of wind tunnel tests performed on different structures.

\subsection{Extraction of aerodynamic derivatives and rational function approximation}

\subsubsection{Free and forced vibration techniques}

Aerodynamic unsteady forces or motion-induced forces are assumed to be related to the aerodynamic derivatives and their linear combination of displacements and their first time derivatives. There are two main approaches (Tamura and Kareem, 2013; Xu, 2013) to determine the aerodynamic derivatives for a section: first, with direct measurements of aerodynamic force components by strain measurements on sectional model when the section is given some forcing (Matsumoto et al., 1997) and second, calculating the force indirectly by measuring unsteady surface pressure and phase difference from the 
Table 4. Different CFD approaches based on FVM and FEM used to study aerodynamic phenomena

\begin{tabular}{|c|c|c|}
\hline Structure & Phenomena & Reference \\
\hline NACA-0012 & $D, R$ & Maîytre et al. (2003) \\
\hline Rectangular, $B / D=8$ & $\mathrm{D}$ & Thiesemann et al. (2003) \\
\hline Great Belt, 200 mm & D & Thiesemann and Starossek (2003) \\
\hline Millau viaduct, 200 mm & D & Thiesemann and Starossek (2003) \\
\hline Tacoma Narrows, 200 mm & D & Thiesemann and Starossek (2003) \\
\hline Slotted rectangular, $450 \mathrm{~mm}$ & $P, D$ & Shirai and Ueda (2003) \\
\hline Slotted flat box, $370 \mathrm{~mm}$ & $P, D$ & Shirai and Ueda (2003) \\
\hline Great Belt & C, D & Vairo (2003) \\
\hline Normandy & C, D & Vairo (2003) \\
\hline$\pi$-section with $B / D=6$ & $A_{2}^{*}$ & Diogo et al. (2005) \\
\hline Great Belt & $D$ & Stærdahl et al. (2007) \\
\hline Hume, $445 \times 33.4 \mathrm{~mm}$ & $D, F$ & Xin and Ou (2007) \\
\hline Slotted box girder & $c$ & Watanabe and Fumoto (2008) \\
\hline Great Belt & $\mathrm{F}$ & Kalesar and Ali (2008) \\
\hline Tacoma & $\mathrm{F}$ & Kalesar and Ali (2008) \\
\hline Box girder, $B / D=11.6$ & $C, D, F$ & Sarwar et al. (2008) \\
\hline Rectangular, $B / D=4$ & $C, D$ & Sun et al. (2008) \\
\hline Rectangular, $B / D=4$ & C, D & Sun et al. (2009) \\
\hline Flat plate & D & Szabó and Györgyi (2009a) \\
\hline Bridge section & D & Szabó and Györgyi (2009a) \\
\hline Incheon, 200 × 30 mm & D & Szabó and Györgyi (2009b) \\
\hline Nine bridge decks, $200 \mathrm{~mm}$ & $D, R$ & Starossek et al. (2009) \\
\hline Five bridge sections & $\mathrm{D}$ & Huang et al. (2009) \\
\hline Hume, $445 \times 33.4 \mathrm{~mm}$ & $D, F$ & Xin et al. (2010) \\
\hline Circular cylinder & $\mathrm{C}$ & Brusiani et al. (2010) \\
\hline Great Belt, $375 \times 54.7 \mathrm{~mm}$ & $\mathrm{D}$ & Brusiani et al. (2010) \\
\hline NACA-0012 & C, D & Bai et al. (2010) \\
\hline U-shape, G1, G2 & $C, D$ & Bai et al. (2010) \\
\hline Four generic sections & $C, D, L$ & Szabó and Györgyi (2011) \\
\hline Thin flat plate, $700 \times 3.5 \mathrm{~mm}$ & $\mathrm{D}$ & Haung and Liao (2011) \\
\hline Second Nanjing Yangtze, $772 \times 63 \mathrm{~mm}$ & $\mathrm{D}$ & Haung and Liao (2011) \\
\hline Bridge deck, $369 \times 60 \mathrm{~mm}$ & $P, C, D$ & Šarkić et al. (2011) \\
\hline Bridge deck, $366 \times 66 \mathrm{~mm}$ & $P, C, D$ & Šarkić et al. (2012) \\
\hline Rectangular, $300 \times 60 \mathrm{~mm}$ & $\mathrm{~F}$ & Liua et al. (2012) \\
\hline Streamlined, $404 \times 60 \mathrm{~mm}$ & $\mathrm{~F}$ & Liua et al. (2012) \\
\hline Great Belt, 31 × 4.34 m & $D, F$ & Zhan et al. (2013) \\
\hline Circular cylinder & C & Brusiani et al. (2013) \\
\hline Great Belt, $375 \times 54.7 \mathrm{~mm}$ & $\mathrm{D}$ & Brusiani et al. (2010) \\
\hline$R-5,8,10,12 \cdot 5,15,20$ & $C, D, F$ & Miranda et al. (2014) \\
\hline Flat plate, $1000 \times 10 \mathrm{~mm}$ & $P, D$ & Ketong and Aiping (2014) \\
\hline Great Belt, $31 \times 4.4 \mathrm{~m}$ & $P, D$ & Ketong and Aiping (2014) \\
\hline Bridge deck, $366 \times 66 \mathrm{~mm}$ & $P, C, D$ & Šarkić (2014) \\
\hline Bridge deck, $366 \times 66 \mathrm{~mm}$ & $P, C, D$ & Šarkić et al. (2015) \\
\hline Rectangular cylinder, $B / D=4$ & C, D & Nieto et al. (2015a) \\
\hline Rectangular cylinder, $B / D=4.9$ & C, D & Nieto et al. (2015b) \\
\hline G1 section & $C, D$ & Nieto et al. (2015b) \\
\hline Six deck sections, $366 \times 66 \mathrm{~mm}$ & $R, C, D, F$ & Patruno (2015) \\
\hline
\end{tabular}

F, flutter limit; D, aerodynamic derivative; C, static wind coefficients; P, pressure; R, Reynolds number dependence

flow-induced motion of the model (Scanlan and Tomko, 1971). The direct measurement usually requires more complex experimental set-ups.

The motion of the structure in the flow induces aerodynamic forces which are characterised by aerodynamic derivatives. These aerodynamic derivatives are commonly measured from wind tunnel tests by using different methods such as free vibration, forced vibration and buffeting tests. In forced vibration tests the scaled deck section is forced to move with specific displacement and velocities (or angular rotations and angular velocities) and the aerodynamic forces are measured, from which aerodynamic derivatives are extracted. Free vibration tests are more common, where the decay in system damping and the change in system stiffness is measured when a deck section model is given some initial displacement. The 
Table 5. Different CFD approaches based on the vortex method used to study aerodynamic phenomena

\begin{tabular}{|c|c|c|}
\hline Structure & Phenomena & Reference \\
\hline Great Belt, $31000 \times 4337 \mathrm{~mm}$ & $P, C, D, F$ & Walther (1994) \\
\hline Great Belt & $D, F$ & Larsen and Walther (1996) \\
\hline Flat plate, $B / D=200$ & D & Walther and Larsen (1997) \\
\hline Tacoma Narrows, $12 \times 2.4 \mathrm{~m}$ & $C, D$ & Larsen and Walther (1997a, 1997b) \\
\hline Great Belt, $31 \times 4.4$ & $C, D$ & Larsen and Walther (1997a, 1997b) \\
\hline Approach span, $25.8 \times 7 \mathrm{~m}$ & C, D & Larsen and Walther (1997a, 1997b) \\
\hline Gibraltar APP, $65 \times 2.5 \mathrm{~m}$ & $C, D$ & Larsen and Walther (1997a) \\
\hline Five generic sections, G1-G5 & $C, D$ & Larsen and Walther (1998) \\
\hline Five generic sections, G1-G5 & $C, D$ & Larsen (1998) \\
\hline Great Belt, $31 \times 4 \mathrm{~m}$ & $D, F$ & Morgenthal (2000) \\
\hline Flat plate, $B / D=100$ & $D, F$ & Morgenthal (2002) \\
\hline Millau viaduct, $388 \times 60.2 \mathrm{~mm}$ & $D, F$ & Morgenthal (2002) \\
\hline Great Belt, $31 \times 4 \mathrm{~m}$ & $C, D$ & Morgenthal and McRobie (2002) \\
\hline H-shaped & $\mathrm{C}$ & Larsen and Walther (2003) \\
\hline Humen and Great Belt & C & Zhou et al. (2003) \\
\hline Nanjing and Runyang & C & Zhou et al. (2003) \\
\hline Jinsha & $D, F$ & Zhou et al. (2003) \\
\hline Tacoma Narrows & $C, A_{2}^{*}$ & Zhou et al. (2006) \\
\hline Stonecutters & $C, D, F$ & Janjic et al. (2008) \\
\hline Tacoma Narrows & $A_{2}^{*}$ & McRobie et al. (2013) \\
\hline
\end{tabular}

F, flutter limit; D, aerodynamic derivative; C, static wind coefficients; P, pressure, R, Reynolds number dependence

buffeting tests are simple, but the measurements need to be treated to remove buffeting forces considered as noise, which requires more advanced system identification techniques.

Some advancement has been made in the efficacy of a customarily used identification procedure (Chen and Kareem, 2004), as well as using ANN algorithms, to predict flutter derivatives (Chen et al., 2008) and equivalent Theodorsen functions (Matsumoto et al., 1993). Free vibration tests are usually performed with coupled 2DOF motion in heave and pitch (Brownjohn and Jakobsen, 2001; Gu et al., 2000; Xu et al., 2014; Zhu et al., 2007); however, this becomes more challenging for 3DOF systems (Chen et al., 2002; Chowdhury and Sarkar, 2003; Xu et al., 2012). The response is treated with the general least-squares theory (Chen et al., 2002), the iterative least-squares method (Chowdhury and Sarkar, 2003), the modified unified least-squares method (Bartoli et al., 2009; Xu et al., 2012) or the weighting ensemble least-squares method (Li et al., 2003) to compute the aerodynamic derivatives. Figure 9 shows a least-squares fit on a force-time history in a forced vibration simulation. Classical forced vibration tests are conducted on decoupled motion to compute aerodynamic derivatives. However, this decoupled motion provides aerodynamic derivatives where the direct components of aeroelastic motion are dominant over the interactive components $(\mathrm{Xu}$ et al., 2014). In addition to the uncoupled techniques, coupled forced vibration techniques are also used to determine aerodynamic derivatives (Haung and Liao, 2011; Larsen et al., 2011; Neuhaus et al., 2009; Wu et al., 2006). Qin and Gu (2004), Gu and Qin (2004), Boonyapinyo and Janesupasaeree (2009) and
Boonyapinyo et al. (2009) used the covariance-driven stochastic subspace identification (SSI-COV) technique, which treats the buffeting forces and responses as input in the analysis rather than noise. Moreover, Boonyapinyo and Janesupasaeree (2010) also introduced the data-driven stochastic subspace identification (SSI-Data) technique. Some efforts have been made to use very small-scale wind tunnel testing to obtain aerodynamic derivatives (Hunyadi, 2009; Piña and Caracoglia, 2009). Some wind tunnel tests performed on different structures are summarised in Table 6 using free, forced and buffeting approaches to compute aerodynamic derivatives.

Aerodynamic derivatives are determined at only some fixed values of amplitudes of oscillation, considering them amplitude independent. However, some studies (Scanlan, 1997a) have found the aerodynamic derivatives to be slightly amplitude dependent. Table 7 presents some examples of forced vibration analyses on different structures and the corresponding displacement amplitudes used. Walther (1994) also suggested the amplitudes of vertical displacement, $h_{\mathrm{o}}$, and rotational displacements, $\alpha_{\mathrm{o}}$, for forced oscillation simulations

11a. $h_{\mathrm{o}}<\frac{1}{\omega} \sqrt{\frac{2 v}{\Delta t}}=\frac{B}{2 \pi}\left(\frac{v_{\mathrm{r}}}{U_{\infty}}\right) \sqrt{\frac{2 v}{\Delta t}}$

11b. $\alpha_{\mathrm{o}}<\frac{1}{\omega} \sqrt{\frac{8 v}{\Delta t}}=\frac{B}{2 \pi}\left(\frac{v_{\mathrm{r}}}{U_{\infty}}\right) \sqrt{\frac{8 v}{\Delta t}}$ 


\begin{tabular}{|c|c|c|c|}
\hline \multirow[b]{2}{*}{ Structure } & \multicolumn{2}{|l|}{ Size } & \multirow[b]{2}{*}{ Reference } \\
\hline & $\begin{array}{l}B \times \mathrm{D}: \mathrm{mm} \\
(B / \mathrm{D})\end{array}$ & Response & \\
\hline Rectangular & $200 \times 20$ & Coupled-forced & Matsumoto et al. (1993) \\
\hline Rectangular & $200 \times 10$ & Coupled-forced & Matsumoto et al. (1993) \\
\hline Box girder & $292.7 \times 33.7$ & Free & Iwamoto and Fujino (1995) \\
\hline Flat plate & $450 \times 20$ & Free & Gu et al. (2000) \\
\hline Jiangyin, 1:70 & $36.9 \times 3.0 \mathrm{~m}$ & Free & Gu et al. (2000) \\
\hline Streamlined & - & Free, buffeting & Brownjohn and Jakobsen (2001) \\
\hline Dual carriageway & - & Free, buffeting & Brownjohn and Jakobsen (2001) \\
\hline Plate, 2DOF, 3DOF & $460 \times 45$ & Free & Chen et al. (2002) \\
\hline Streamlined box & $450 \times 32$ & Free & Chen et al. (2002) \\
\hline P-shaped & $452 \times 46$ & Free & Chen et al. (2002) \\
\hline NACA-0020, 3DOF & 314 & Free & Chowdhury and Sarkar (2003) \\
\hline Chamfered plate & (25) & Forced & Wu et al. (2006) \\
\hline Rectangle & $(5,8,10,12 \cdot 5,15,20)$ & Free & Chen et al. (2008) \\
\hline NACA-0020 & 456 & Forced & Neuhaus et al. (2009) \\
\hline Tacoma Narrows & $411 \times 82$ & Forced & Neuhaus et al. (2009) \\
\hline Strelasund & $324 \times 103$ & Forced & Neuhaus et al. (2009) \\
\hline Model A, 1:250 & $123 \times 13$ & Free, forced & Piña and Caracoglia (2009) \\
\hline Model B, 1:500 & $61.33 \times 6.35$ & Free, forced & Piña and Caracoglia (2009) \\
\hline Extradosed, 1:100 & $295 \cdot 4$ & Forced & Hunyadi (2009) \\
\hline Flat plate & $450 \times 20$ & Free & Boonyapinyo and Janesupasaeree (2009) \\
\hline Industrial ring road & $399 \times 35$ & Free & Boonyapinyo and Janesupasaeree (2009) \\
\hline Industrial ring road & $399 \times 35$ & Free & Boonyapinyo et al. (2009) \\
\hline Fairing - modified, 1:90 & $399 \times 35$ & Free & Boonyapinyo et al. (2009) \\
\hline Soffit plate modified & $399 \times 35$ & Free & Boonyapinyo et al. (2009) \\
\hline Combination of two & $399 \times 35$ & Free & Boonyapinyo et al. (2009) \\
\hline Single-box & $450 \times 70$ & Free & Bartoli et al. (2009) \\
\hline Multiple-box girder & $609 \times 52$ & Free & Bartoli et al. (2009) \\
\hline Great Belt, 1:60 & $31 \times 4 m$ & Free & Larsen et al. (2011) \\
\hline Sutong & 812 & Free & Xu et al. (2012) \\
\hline Suramadu & 600 & Free & Xu et al. (2012) \\
\hline Flat plate & - & Free & Xu et al. (2014) \\
\hline Sutong & $41 \times 4 \mathrm{~m}$ & Free & Xu et al. (2014) \\
\hline Stonecutters & $53 \cdot 3 \times 4 \mathrm{~m}$ & Free & Xu et al. (2014) \\
\hline Akashi-Kaikyo & $35.5 \times 14 \mathrm{~m}$ & Free & Xu et al. (2014) \\
\hline
\end{tabular}

where $\omega$ is the oscillation frequency, $v$ is the kinematic viscosity, $v_{\mathrm{r}}$ is the reduced speed and $\Delta t$ is the time step.

The aerodynamic derivatives are also influenced by the wind angle of attack and are usually determined for several angles (Jurado et al., 2008; Mannini and Bartoli, 2008). Zhu et al. (2002, 2006) and Huang et al. (2012) found the notable dependence of the flutter limit of a deck section under wind inclination and yaw angle.

\subsubsection{Kernel identification procedures}

The identification of kernel functions describing unsteady aerodynamics, linear or non-linear, is one of the major tasks when a time-domain analysis is performed. Commonly, these are the indicial and impulse functions described previously. The indicial functions can be obtained directly based on experiments (Caracoglia and Jones, 2003a; Yoshimura and Nakamura, 1979) or by a CFD approach (Brar et al., 1996; Farsani et al., 2014; Miranda et al., 2013; Turbelin and Gibert, 1996); however, these methods require additional refinement to be used as a simple identification scheme (Wu and Kareem, 2014b). Commonly, for bluff bodies they are most often obtained by performing the Fourier transform of the exponential kernel function and equating to the flutter derivatives (Caracoglia and Jones, 2003a, 2003b; Scanlan, 1984; Scanlan et al., 1974). The impulse function can also be related to the aerodynamic derivatives using the same analogy (Bucher and Lin, 1988) as shown in Table 8 for the lift force due to vertical displacement and rotation. The rest of the terms can be obtained accordingly. The indicial function for a flat plate was approximated by a set of exponential functions through operational calculus (Jones, 1940), commonly known as Wagner-like functions. Generally, in both impulse and indicial functions, the unsteady terms are approximated using exponential functions, which are later on convoluted, while the 


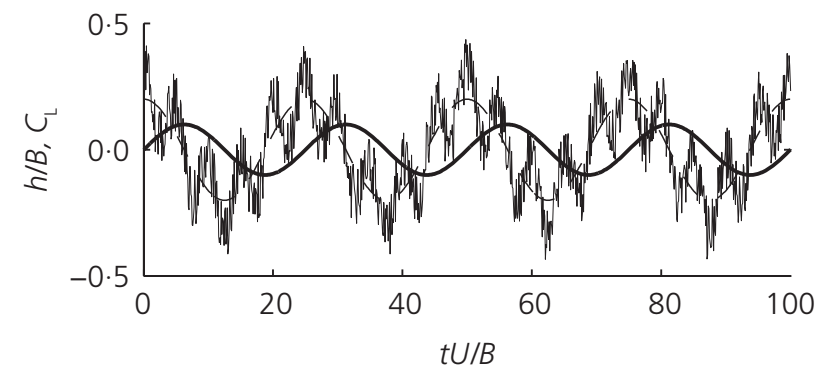

Figure 9. Forced oscillation simulation: least-squares-fit on lift (or moment) coefficient time traces to calculate aerodynamic derivatives $(-$, forced displacement $h / B$ (or rotation $\alpha) ;-$, measured lift $C_{\mathrm{L}}$ (or moment $C_{\mathrm{M}}$ ); - -, least-squares-fit on lift (or moment)) quasi-steady values are obtained from the linearly dependent terms of the velocity and displacements. The coefficients of the exponential kernels in the indicial function approach for different types of streamlined and bluff bridge decks are obtained through using rational approximation by applying the non-linear leastsquares method (Borri and Höffer, 2000; Caracoglia and Jones, 2003a, 2003b; Costa, 2007; Costa and Borri, 2006; Scanlan, 1984), while for the impulse function a bi-level optimisation scheme utilising the linear least-squares and Nelder-Mead algorithm is used, as described by Tiffany and Adams $(1984,1988)$.

An in-depth description of the identification procedure for the indicial functions was provided recently by Zhang et al. (2011),

Table 7. Amplitudes of heave and pitch used for forced vibration tests

\begin{tabular}{|c|c|c|c|c|}
\hline \multirow[b]{3}{*}{ Structure } & \multirow{3}{*}{$\begin{array}{l}\text { Size } \\
B \times \mathrm{D}: \mathrm{mm} \\
(B / D)\end{array}$} & \multirow{3}{*}{$\begin{array}{l}\text { Heave amplitude } \\
\frac{h_{\mathrm{o}} / B}{\left(h_{\mathrm{o}}: \mathrm{mm}\right)}\end{array}$} & \multirow{3}{*}{$\begin{array}{l}\text { Pitch amplitude } \\
\alpha_{\mathrm{o}} \text { : deg }\end{array}$} & \multirow[b]{3}{*}{ Reference } \\
\hline & & & & \\
\hline & & & & \\
\hline Rectangular & $200 \times 20$ & $h_{\mathrm{o}} / \alpha_{\mathrm{o}}=0.0637$ & & Matsumoto et al. (1993) \\
\hline Rectangular & $200 \times 10$ & $h_{\mathrm{o}} / \alpha_{\mathrm{o}}=0.1408$ & & Matsumoto et al. (1993) \\
\hline Great Belt & 31000 & 0.05 & 5 & Walther (1994) \\
\hline Five generic & - & 0.05 & 3 & Larsen (1998) \\
\hline sections, G1-G5 & - & 0.05 & 3 & Larsen and Walther (1998) \\
\hline Flat plate & $(100)$ & 0.50 & 3 & Morgenthal (2002) \\
\hline Great Belt & 200 & 0.04 & 5 & Thiesemann and Starossek (2003) \\
\hline Tacoma Narrows & 200 & 0.04 & 5 & Thiesemann and Starossek (2003) \\
\hline Rectangular & 200 & 0.04 & 5 & Thiesemann and Starossek (2003) \\
\hline Rectangular & $200(8)$ & 0.04 & 8 & Thiesemann et al. (2003) \\
\hline Great Belt & 200 & 0.04 & 8 & Thiesemann et al. (2003) \\
\hline Millau viaduct & 200 & 0.04 & 8 & Thiesemann et al. (2003) \\
\hline Tacoma Narrows & 200 & 0.04 & 8 & Thiesemann et al. (2003) \\
\hline$\pi$-section & $12000 \times 2000$ & - & $1 \cdot 72$ & Diogo et al. (2005) \\
\hline Thin plate & $40000(100)$ & $(100)$ & 3 & Ge and Xiang (2008c) \\
\hline H-shape & 11900 & (100) & 3 & Ge and Xiang (2008c) \\
\hline Great Belt & 31000 & (100) & 3 & Ge and Xiang (2008c) \\
\hline PWRI & - & 0.01 & 1 & Sarkar et al. (2009) \\
\hline ISU & 300 & 0.05 & $2 \cdot 8$ & Sarkar et al. (2009) \\
\hline Rectangular & $(2-5)$ & $0.01-0.20$ & $2 \cdot 8$ & Sarkar et al. (2009) \\
\hline Nine bridge decks & 200 & $0.01,0.02,0.04$ & $2,5,8$ & Starossek et al. (2009) \\
\hline Model A, 1:250 & $123 \times 13$ & $(0.5-1.49)$ & $1 \cdot 77-2 \cdot 35$ & Piña and Caracoglia (2009) \\
\hline Model B, 1:500 & $61.33 \times 6.35$ & $(5-6)$ & $3 \cdot 43$ & Piña and Caracoglia (2009) \\
\hline Thin flat plate & $700 \times 3.5$ & $(2 \cdot 5-6 \cdot 12)$ & $0.35-0.86$ & Haung and Liao (2011) \\
\hline Second Nanjing Yangtze & $772 \times 63$ & $(2 \cdot 5-5 \cdot 59)$ & $0.40-0.89$ & Haung and Liao (2011) \\
\hline Great Belt east, $1: 60$ & $31000 \times 4000$ & (16) & 2 & Larsen et al. (2011) \\
\hline Bridge section & $369.5 \times 60 \cdot 6$ & (4) & 1 & Šarkić et al. (2011) \\
\hline Bridge section & $366 \times 66 \cdot 6$ & (4) & 1 & Šarkić et al. (2012) \\
\hline Bridge section & $366 \times 66.6$ & (4) & 1 & Šarkić (2014) \\
\hline Bridge section & $366 \times 66.6$ & (4) & 1 & Šarkić et al. (2015) \\
\hline
\end{tabular}

Table 8. Relations between the Fourier transform of indicial and impulse functions and aerodynamic derivatives (after Caracoglia and Jones (2003b) and Chen and Kareem (2002))

$\begin{array}{lllll}\text { Indicial/Impulse } & \bar{\Phi}_{L h}(k)+\Phi_{L h}(0) & \bar{\Phi}_{L \alpha}(k)+\Phi_{L h}(0) & \bar{l}_{L h}(k) & \bar{l}_{L \alpha}(k) \\ \text { Scanlan } & \frac{K}{C L}\left(H_{1}^{*}(K)-i H_{4}^{*}(K)\right) & \frac{K^{2}}{C L}\left(H_{3}^{*}(K)+i H_{2}^{*}(K)\right) & \frac{K^{2}}{2}\left(H_{4}^{*}(K)+i H_{1}^{*}(K)\right) & \frac{K^{2} b}{2}\left(H_{3}^{*}(K)+i H_{2}^{*}(K)\right)\end{array}$


with the extension of the overshooting phenomenon and its effect on the transient response for Aizhai Bridge. Wilde et al. (1996) compared the different formulations of the impulse functions for bridge decks, as previously used in aeronautics (e.g. Karpel, 1981; Roger, 1977). Although the number of lagterms describing the unsteady behaviour was different, there was no significant difference in the results. In bridge aerodynamics, Guo and Ge (2012) and Liu and Ge (2012) introduced a new formulation in the state-space. On the experimental side, Chowdhury and Sarkar (2005) proposed a new approach to directly identify the exponential coefficients based on wind tunnel testing. A comparative analysis between the approximation of indicial functions, impulse functions and their counterparts in the frequency domain has been done by Øiseth et al. (2011) using a statistical approach on more than 20 time history analyses, resulting in a maximum $3 \%$ deviation of the flutter limit with respect to the frequency domain, proving no apparent superiority between the approximations. Recently, the rheological models have been used as kernels to approximate the linear (Diana et al., 2013) and the non-linear (Diana et al., 2010) unsteady aerodynamic forces. The linear part was obtained conventionally from the flutter derivatives by fitting several independent mechanical oscillators up to second order using constrained optimisation, whereas the non-linear was obtained by approximating the aerodynamic hysteretic behaviour. Liao et al. (2012) used non-linear harmonic oscillators for the approximation of the hysteresis loop representing the non-linear aerodynamic force with higher-order unsteady effects, yielding a rather more simple formulation. Following the same concept, Wu and Kareem (2013) utilised an ANN to model the aerodynamic non-linear behaviour of bluff bodies. The limitation of these models is the difficulties in the physical interpretation of the parameters. For the approximation of the non-linear convolution kernels in the Volterra series, a sparse third-order scheme was identified as sufficient in the case of the Tacoma Narrows Bridge by Wu and Kareem (2014c), which would significantly reduce the computational time that presents a major setback in the Volterra series. This was followed by a framework for kernel identification based on a CFD approach utilising unit-step function by the same authors (Wu and Kareem, 2015). The comparative analysis of the first- and higher-order kernels and their amplitude dependence showed the mathematical superiority of this model for simulating non-linear bridge aerodynamics.

\section{Flutter limit prediction}

\subsection{Fully analytical - Theodorsen theory}

The analytical formulation of aeroelastic instability problems initially came from the aeronautical industry, especially for the solution of flutter problem of aeroplane wings. Theodorsen (1935) gave an analytical solution based on potential flow theory for the motion-induced aerodynamic forces on a flat plate. A comparison of experimental data with the theoretical solution was made by Theodorsen and Garrick (1940). The phenomenon of flutter is characterised by coupling of oscillatory heave and pitch harmonic motion when the frequencies of these two modes coincide. Analytical flutter analysis requires simultaneous solution of the generalised equations of motion of a $2 \mathrm{DOF}$ system as a complex eigenvalue problem. Often only the first fundamental heave mode and the first fundamental torsional mode are considered, for which a simplified 2D flutter analysis can be performed. This simplification assumes that only these selected modes participate in the flutter analysis, which may lead to overestimation of the flutter limit. On the one hand, this approach is independent of the shape of the body but, on the other hand, it neglects the effects originating from the simplification to a flat plate; therefore, this analysis is only valid for a flat plate or an aerofoil. The solution of an eigenvalue problem using the Theodorsen equation of motion for heave and pitch can be found in Abbas (2011, 2016), Abbas and Morgenthal (2012a, 2012b) and Morgenthal (2002).

\subsection{Empirical approach}

Bleich (1948) introduced an empirical formula using Theodorsen's flutter theory for a flat plate to calculate the critical flutter limit. Selberg (1961) also developed a closed-form solution by introducing the shape ratio to apply for various types of bridge cross-sections. Later Klöppel and Thiele (1967) gave a formula that is suitable for hand calculations by making use of empirical diagrams (Svensson, 2013).

The theory of thin aerofoils helps to explain the aeroelastic phenomena of torsional divergence. The wind velocity, called the divergence velocity $U_{\mathrm{d}}$, corresponds to the occurrence of a static stability problem, as even the smallest rotation of the aerofoil from the neutral position will imply displacements without limits. The divergence wind speed can be calculated by using the aerodynamic moment coefficient $C_{\mathrm{M}}$ of the deck for varying wind angles of attack $\theta$ as follows

12. $U_{\mathrm{d}}=\sqrt{\frac{2 K_{\alpha}}{\rho B^{2} C_{\mathrm{M}}^{\prime}}}=B \omega_{\alpha} \sqrt{\frac{2 I}{\rho B^{4} C_{\mathrm{M}}^{\prime}}}$

where $K_{\alpha}$ is the torsional stiffness of the deck. It should be noted that the divergence velocity from the above equation is only valid when the aerodynamic moment coefficient is linear for the angles of attack of interest.

It is usual that the flutter limit $U_{\mathrm{cr}}$ is lower than the divergence wind speed $U_{\mathrm{d}}$ for cable-supported bridges, which makes static instability of less interest than flutter (Gimsing and Georgakis, 2011). Generally, the torsional frequency $f_{\alpha}$ is larger than the vertical frequency $f_{h}$ in the case of cable-supported bridges 
with two cable planes; however, the torsional frequency may decrease with increasing wind speed due to the equivalent reduction of the torsional stiffness under the action of the aerostatic pressure. Frandsen (1966) showed that this could lead to the flutter limit for the aerofoil as

13. $U_{\mathrm{cr}}=U_{\mathrm{d}} \sqrt{1-\left(\frac{\omega_{h}}{\omega_{\alpha}}\right)^{2}}$

It is clear from the above equation that the flutter limit is smaller than the divergence velocity. It also appears that flutter will happen at lower wind velocity if the vertical and the torsional frequencies are close. Therefore, it is generally desired to keep the frequency ratio $f_{\alpha} / f_{h}$ more than two in larger cable-supported bridges (Gimsing and Georgakis, 2011). The above expression agrees reasonably well with the semiempirical expression by Selberg (1961), who gave the following expression which is based on a theoretical thin aerofoil for the flutter limit (Tamura and Kareem, 2013)

14. $U_{\mathrm{cr}}=3.7 B f_{\alpha} \sqrt{\frac{m \sqrt{I / m}}{\rho B^{3}}\left[1-\left(\frac{\omega_{h}}{\omega_{\alpha}}\right)^{2}\right]}$

Selberg's expression is found in slightly different form in Bartoli and Mannini (2008), Gimsing and Georgakis (2011), Holmes (2015) and Strømmen (2006). Selberg's formula may be used to provide a first estimate of the flutter stability limit for a flat plate where frequency ratio $f_{\alpha} l f_{h}>1 \cdot 5$ (Strømmen, 2006).

Rocard reported very similar empirical formula (Bartoli and Mannini, 2008; Frandsen, 1966); with the notation adopted in the present paper, this formula is as follows

15. $U_{\mathrm{cr}}=6 \cdot 282 f_{\alpha} B \sqrt{\left(1-\frac{1}{\gamma_{\omega}^{2}}\right) \frac{r_{\alpha}^{2} \mu}{1+8 r_{\alpha}^{2}}}$

where

16. $\quad \mu=\pi \rho B^{2} / 4 m ; \quad r_{\alpha}=\sqrt{\frac{I}{m B^{2}}} ; \quad \gamma_{\omega}=\frac{\omega_{\alpha}}{\omega_{h}}$

Matsumoto et al. (2008a) also gave a simplified formula to calculate the flutter limit for a flat plate using relationships between mass $m$ and mass moment of inertia $I$ as $I=m b^{2} / 3$, which is very similar to Selberg's form as follows

17. $U_{\mathrm{cr}}=3 \cdot 81 B f_{\alpha} \sqrt{\frac{\sqrt{I m}}{\rho B^{3}}\left[1-\left(\frac{\omega_{h}}{\omega_{\alpha}}\right)^{2}\right]}$
Another simplified formula which is well known for its simplicity and convenience was given by Put (1976) to calculate the critical flutter limit as follows

18. $U_{\mathrm{cr}}=\eta\left[1+\left(\frac{\omega_{\alpha}}{\omega_{h}}-0.5\right) \sqrt{\frac{r}{b} 0 \cdot 72 \mu}\right] \omega_{h} b$

where $r=(I / m)^{1 / 2}$ is the radius of gyration and $\eta$ is an empirical form factor giving the difference of the critical wind speed of a certain profile with respect to flat plate prediction (1 for a flat plate). The above equation can further be simplified ( $\mathrm{Fu}$ and Wang, 2014) as follows

19. $U_{\mathrm{cr}}=\left(2 \cdot 5 \sqrt{\frac{r}{b} \mu}\right) 2 b f_{\alpha}$

A wide streamlined deck of a bridge shows usually a critical velocity close to the theoretical flat plate flutter limit, whereas bluff girders, trusses and deep deck sections show a larger difference between the theoretical flutter limit and that measured in the wind tunnel. Usually, this difference is represented with the help of a ratio of measured flutter limit to flat plate prediction, $\beta_{\mathrm{f}}$, which is about $90 \%$ for a streamlined box girder and can be up to $40 \%$ for bluff box sections (Gimsing and Georgakis, 2011). It would appear to be unwise to rely on a prediction based on an empirical formula alone; therefore, more detailed analyses are required for important structures and at the final design stage.

The Eurocode (BSI, 2005) requires the avoidance of divergence and flutter where Equation 12 is considered to calculate the critical wind velocity for divergence. The assessment of the susceptibility of a structure is provided in terms of simple structural criteria. The structure needs to be checked for three criteria: the aspect ratio $D / B<0 \cdot 25$, the torsional axis normal to wind direction with the centre of torsion at least $B / 4$ downwind of the windward edge of the section and the frequency ratio $f_{\alpha} / f_{h}<2$. It recommends specialist advice in cases where these criteria are met.

\subsection{Semi-analytical/derivative-based analysis}

\subsubsection{Solution in frequency domain}

The flutter analysis is traditionally conducted in the frequency domain as the aerodynamic derivatives are functions of reduced speed, which requires an iterative solution to determine the flutter limit. Commonly, the iterative solution is performed by complex eigenvalue analysis. There are two approaches that can be followed to perform these calculations (Wenzel, 2008): the first is the direct method, where the unsteady aerodynamic forces are applied to the finite-element 
model, either in the frequency domain or in the time domain; the second is the mode superposition method where the structural response is separately considered in various vibration modes and to assemble them.

Flutter analysis is preferred to be performed in the frequency domain for its computational efficiency because aerodynamic derivatives in the motion-induced forces are functions of reduced frequency $(\mathrm{Xu}, 2013)$. There are two main analytical approaches for the solution of flutter problems: the complex eigenvalue analysis (Ceva) (Simiu and Scanlan, 1996) and the step-by-step analysis (SBSA) (Matsumoto, 1996). The Ceva has been used for a long time for flutter problems. In the Ceva, the main challenge is related to the investigation of the system frequency and damping ratio relationship with the wind speed, whereas the SBSA is very useful for such limitations. Additionally, it describes the role of flutter derivatives in the critical condition and in flutter stabilisation.

Motion-induced aerodynamic forces depend on the response frequency of the deck, which is determined in the analysis of a complex non-linear eigenvalue problem and then the critical flutter limit is calculated (Hernández et al., 2008; Jurado and Hernández, 2004). An iterative solution is necessary because the aerodynamic derivatives depend on the reduced speed $\left(v_{\mathrm{r}}=2 \pi U / B f\right)$, which is based on the oscillation frequency, $f$, which needs to be known. Xu (2013) used Ceva and computed the flutter limit of the Stonecutters Bridge. He also showed the dynamic characteristics and aerodynamic derivatives of the bridge deck with and without traffic. Dyrbye and Hansen (1997) presented modal-form solutions where the real and imaginary parts with mode shape similarity factors were given. Dung et al. (1998) traced the system complex mode with the increasing wind speed, which helps to describe the dynamic behaviour before flutter.

Matsumoto et al. $(1995,1996)$ and Matsumoto (1996) developed the SBSA and reported flutter characteristics including frequency, damping, amplitude ratio and phase difference characteristics and then compared these with the Ceva, which showed reasonable agreement. Discrepancies were observed in the results obtained by the Ceva and the SBSA at higher reduced speeds beyond the flutter limit; therefore, both the damping and flutter frequency have to converge simultaneously in the iteration (Matsumoto et al., 2007, 2010). Later modifications showed perfect agreement (Matsumoto et al., 2007, 2010). In other studies (Matsumoto et al., 1998, 1999, 2006a, 2006b, 2008b, 2009) the complex branch switch characteristics of coupled flutter instability are the main focus. This method was applied to highlight the characteristics of different bluff sections (Matsumoto et al., 2002, 2008c). Yang et al. $(2006,2007,2009)$ and Ge and Xiang (2008a) also proposed, based on the concept of SBSA analysis, a 2D coupled flutter analysis (CFA) for 3DOF to investigate the mechanism of flutter, with the focus on the aerodynamic damping, frequency and flutter modality. Chen (2007) presented closedform formulations for estimating the modal frequencies, modal damping ratios and coupled motions of a $2 \mathrm{D}$ aeroelastic bridge with variation in the wind speed.

The two branches in coupled flutter are known as the heaving branch (HB) and the torsional branch (TB). In the SBSA, the flutter frequency in the $\mathrm{HB}$ and the TB are converged by iterative calculation. Based on several fundamental sections and flow-structure interaction, Matsumoto and co-workers also classified the flutter generation mechanism into detailed branches (Matsumoto et al., 1999, 2002, 2003, 2007, 2008a, 2008b, 2008c)
- low-speed torsional flutter
- high-speed torsional flutter
- heaving-branch coupled flutter
- torsional-branch coupled flutter
- heaving-torsional coupled flutter.

\subsubsection{Solution in time domain}

The frequency-domain approach for determining the flutter limit is limited to considering only a linear system under the action of the stationary wind without taking into account aerodynamic or structural non-linearities. Therefore, a major part of recent research focuses on the time-domain analysis, where it is possible to include these non-linearities. One of the earliest implementations was by Beliveau et al. (1977), utilising the indicial function for the time and frequency domain. Initially, the buffeting analysis was usually conducted in the time domain using the quasi-steady approach (Diana et al., 1993; Kovacs et al., 1992; Miyata et al., 1995a, 1995b) with a statespace model for impulse functions (Xiang et al., 1995). Boonyapinyo et al. (1999), Chen et al. (2000a, 2000b, 2001), Chen and Kareem (2001a), Thang et al. (2008), Øiseth et al. (2011, 2012) and Guo and Ge (2012) presented a time-domain buffeting and flutter analysis based on the state-space using rational approximation of the impulse functions for full and reduced models in modal coordinates.

In state-space formulation, the convolution is described by a linear superposition of additional aerodynamic states representing the fluid memories. The integration is then carried out using first-order time integration schemes such as RungeKutta or Euler. The flutter limit is then compared to conventional frequency analysis. Chen et al. (2000b) presented a full model where additional iterations were required for each step in the Newmark-beta algorithm. From the solution of secondorder differential equations, Lazzari et al. (2004) investigated the convulsion integrals over the impulse functions and proposed a scheme for reduced integration for a finite time for 
the convolution. Both schemes proved to be successful in predicting the flutter limit for a cross-sectional model. Borri et al. (2002), Costa and Borri (2006), Costa (2007), Salvatori and Borri (2007), Zhang et al. (2003a), Zhang and Chen (2010) and Kurniawan (2013) determined the flutter limit based on an approximation of the indicial functions for a full model by integrating the convolution integral each time-step. To reduce the computational cost, Borri and Höffer (2000) presented a time window for the finite convolution of the indicial function, suggesting that only recent time has an impact on the rise of the self-excited forces.

The quasi-steady theory was applied by Salvatori and Spinelli (2007), Borri and Costa (2004) and Øiseth et al. (2010) in the determination of the flutter limit, utilising its features to capture LCO. Chen and Kareem (2000, 2001b, 2003a) proposed a model considering the aerodynamic non-linearities by utilising the quasi-steady theory for high reduced velocities capturing the non-linear part and the impulse-function-based model for the low reduced velocities for the linear unsteady effects. In the case of laminar flow, this constitutes a linearisation about the non-linear aerostatic angle of incidence. A similar method, referred to as the band-superposition scheme, was used by Diana et al. (2002), splitting the wind frequency into several bands; however, there was no clear link between the bands and it was not used in order to determine a flutter wind velocity. In Diana et al. (2013), the bandsuperposition model was extended by using the corrected quasi-steady model for the lower frequency part and the linear unsteady model for the high-frequency components. The most recent models (Diana et al., 2010, 2013; Wang and Liao, 2012; $\mathrm{Wu}$ and Kareem, 2011) attempted to determine the flutter limit including the aerodynamic non-linearity and the nonlinear memory terms based on the hysteretic behaviour. Wu and Kareem (2013) gave a comparison between the models and methods for computing the flutter limit, discussing the influence of the aerodynamic non-linearity and linear and nonlinear fluid memory. Further comparisons for time-domain flutter analysis were done by Lazzari (2005) and Petrini et al. (2007).

\subsubsection{Simplified approach}

Some attempts have been made to simplify flutter analysis by developing the relationship and mutual dependence of aerodynamic derivatives (Matsumoto et al., 1996) such as

20a. $H_{1}^{*}=k H_{3}^{*} ; \quad H_{4}^{*}=-k H_{2}^{*}$

20b. $\quad A_{1}^{*}=k A_{3}^{*} ; \quad A_{4}^{*}=-k A_{2}^{*}$
Furthermore, there is another dependence between two pairs $\left(A_{2}^{*}-A_{3}^{*}\right)$ and $\left(H_{1}^{*}-H_{4}^{*}\right)$ because of the equivalent Wagner function. This concludes that there are only two independent aerodynamic derivatives among the eight. Scanlan et al. (1997) analytically described the interrelation and the dependence among the aerodynamic derivatives of an aerofoil and investigated experimentally obtained aerodynamic derivatives of three different bridge deck sections. It was found that this relationship clearly does not hold for bluff sections. However, $\mathrm{Xu}$ (2009a, 2009b) investigated the relationship between aerodynamic derivatives of slender bridges and, based on his previously proposed semi-analytical aerodynamic derivatives of flexible structure, showed that certain relationships exist between these derivatives as follows

21a. $H_{1}^{*}=-4 A_{1}^{*} ; \quad H_{2}^{*}=-4 A_{2}^{*}-\frac{\pi}{k}$

21b. $H_{3}^{*}=-4 A_{3}^{*}+\frac{\pi}{16} ; \quad H_{4}^{*}=-4 A_{4}^{*}+\frac{\pi}{2}$

21c. $H_{1}^{*}=k H_{3}^{*}-\frac{\pi G}{2} ; \quad H_{2}^{*}=-\frac{H_{4}^{*}}{k}-\frac{\pi F}{2 k}$

$21 d$.

$A_{1}^{*}=k A_{3}^{*}-\frac{\pi k}{64}+\frac{\pi G}{8} ; \quad A_{4}^{*}=-k A_{2}^{*}-\frac{\pi}{8}(1-F)$

The study also shows that semi-analytical aerodynamic derivatives are applicable to bridges with a streamlined cross-section. Additionally, Tubino (2005) provides the interrelationship among aerodynamic derivatives using generalised quasi-static theory. Al-Assaf (2006) developed an alternative analytical approach to supplement wind tunnel testing to estimate the flutter limit of a bluff bridge section by synthesising the aerodynamic derivatives based on previous studies of a similar deck configuration. Lute et al. (2009) also used the aerodynamic derivative database to predict the flutter derivatives for any deck size for estimation of flutter limit. Mannini (2006) investigated flutter instability by analysing a large number of dynamic and aerodynamic data and proposed a simplified expression which uses only three or even two aerodynamic derivatives for the calculation of the flutter limit (Bartoli and Mannini, 2008; Borri and Mannini, 2010; Mannini et al., 2012). Lee et al. (2011) gave another simplified formula by applying the quasi-steady approach and using approximations in the aerodynamic derivatives for calculating the 2D flutter limit. Øiseth and Sigbjörnsson (2011) presented a simplified analytical formulation, including closed-form 
algebraic expressions, for the prediction of the critical flutter limit. Øiseth et al. (2010) suggested a simplified approach to compute the flutter limit by using a new set of modified quasisteady coefficients. Banerjee (2003) derived expressions for generalised mass, generalised stiffness and generalised aerodynamic force terms in compact explicit form. Vairo (2010) proposed an approach for the dynamic problem by considering a simple mechanical system with equivalent stiffness properties for capturing the main wind-bridge interaction mechanisms. All of these simplifications generally provide a reasonable prediction for flat plate flutter but are not suitable for real bridge crosssections.

\subsubsection{Multi-mode approach}

The study of flutter phenomena is related to the understanding of the nature of unsteady aerodynamic forces, mechanisms of mode-coupling and the methodology of multi-mode analysis (Yamada et al., 2006). Scanlan (1987) and Scanlan and Jones (1990) introduced single-mode and 2D flutter analysis methods using modal coordinates by considering the main contribution of selected fundamental torsional mode (for torsional flutter) and coupling between pitch and heave modes (for classical flutter). The 2D coupled flutter analysis is sometimes not sufficient when the contribution of higher modes is significant. Therefore, the mode-by-mode approach can be adopted (Fujino and Siringoringo, 2013b). A 2D analysis is applicable to a case where the deck is straight, with prismatic cross-section and heave and pitch modes having the same form across the span of the bridge (Tamura and Kareem, 2013). Additionally, it assumes the same wind velocity across the whole deck level.

The consideration of the horizontal modes leads to a 3D flutter analysis (Tanaka et al., 1992). The lateral modes may have a significant influence on the accurate prediction of the flutter limit for long-span bridges (D'Asdia and Sepe, 1998; Mishra et al., 2008a, 2008b). The horizontal movement of the deck along the direction of the wind for very flexible long-span bridges can play an important role, such as in the case of the Akashi Strait Bridge, where Katsuchi et al. $(1998,1999)$ and Yamada et al. (2006) showed that by including $P_{i}^{*}$ aerodynamic derivatives flutter limit reduced considerably. This significant participation of lateral modes in the flutter instability has never been observed before (Hoa, 2008). Later, Matsumoto et al. (2010) showed that it is due to the structural coupling of the lateral modes with the torsional mode. Aerodynamic derivatives $P_{i}^{*}$ are usually not directly measured from wind tunnel tests, therefore, quasi-steady values may be used, as shown in Table 9 .
The state-of-the-art flutter analysis is generally carried out in the frequency domain by using generalised coordinate transformation in modal space sometimes using the FEM. The 3D flutter analysis uses superposition of modes in which dynamic coupling between the structural modes occurs through the selfexcited aerodynamic forces (Astiz, 1998; Hua and Chen, 2008; Nakamura and Ogaki, 1990; Namini et al., 1992; Øiseth et al., 2013; Tamura and Kareem, 2013; Vu et al., 2015). Only a few modes are susceptible to flutter instability which are necessary to consider in the analysis. The selected modes of vibration are included in the flutter analysis, which is an approximation; however, it can give good precision if sufficient modes are chosen. The 3D effects of the structure and the wind characteristics, as well as the influence of coupled modes, are important for flutter stability analysis, as the change in characteristics of the dominant mode influences the critical flutter limit. Scanlan and Jones (1990), Pleif and Batista (1995), Jain et al. (1996b), Katsuchi et al. (1998) and Ge et al. (2002) observed, in some cases, the significant effect of higher modes in the flutter limit prediction instead of considering only fundamental bending and torsional modes. It is possible to have multiple mode coupling at the same critical wind speed when more than one curve representing the real part of the eigenvalues (related to damping) simultaneously, becomes positive (Jurado et al., 2011, 2013). This is very similar to multiple buckling modes of a column under axial load.

Flutter analysis is often performed in the modal space for systems with multiple modes, for which the development of analytical methods has been carried out by Agar $(1989,1991)$. The approaches in the modal space utilise generalised coordinate transform and modal superposition techniques (Hoa, 2006, 2008). Scanlan (1978a, 1978b), Agar (1989), Jain et al. (1996b), Katsuchi et al. (1998), Gu et al. (1999), Ge et al. (2000) and $\mathrm{Vu}$ et al. (2011) have utilised multi-mode approaches to study the flutter response of long-span bridges. Ge and Tanaka (2000a), Ding et al. (2002) and Hua and Chen (2008) examined full-order methods and compared the results with the two-mode and multi-mode procedures. In the multimode approach, selected modes were included in the analysis, whereas the full-order method considered all DOF, which is accurate for flutter analysis from the viewpoint of methodology; however, this approach requires more computation time. Chen and Kareem (2003b, 2003c, 2004, 2006a, 2006b, 2008) provided valuable insight into multi-mode coupled flutter by introducing closed-form expressions without using Ceva for estimating modal characteristics of bridge systems and 2D

Table 9. Conversion between the notation for the aerodynamic derivatives by Scanlan and quasi-steady theory (after Xu (2013))

\begin{tabular}{|c|c|c|c|c|c|c|c|c|}
\hline Scanlan & $P_{1}^{*}$ & $P_{2}^{*}$ & $P_{3}^{*}$ & $P_{5}^{*}$ & $H_{5}^{*}$ & $A_{5}^{*}$ & $H_{6}^{*}, A_{6}^{*}$ & $P_{4}^{*}, P_{6}^{*}$ \\
\hline Quasi-steady & & $\frac{1}{2 K} C_{D}^{\prime}$ & $\frac{1}{2 K^{2}} C_{D}^{\prime}$ & $\frac{1}{2 K} C_{D}^{\prime}$ & $\frac{1}{K} C_{L}$ & $-\frac{1}{K} C_{M}$ & 0 & 0 \\
\hline
\end{tabular}


flutter prediction; they also provided guidance on the selection of critical structural modes and understanding of the multimode coupled bridge flutter response. Zhang et al. (2003b) investigated the effects of each aerodynamic derivative and mode coupling on the flutter limit.

Starossek (1993, 1997, 1998a) used the FEM to model bridge decks using beam elements to predict the flutter limit for a multi-degree-of-freedom (MDOF) system. The complex representation was utilised to predict the $2 \mathrm{D}$ torsional flutter limit (Starossek, 1994) and coupled flutter in the mode space (Starossek, 1998b) by comparing real-number and complexnumber descriptions of the same mechanical phenomena. Thiesemann et al. (2003), Starossek et al. (2009) and Thiesemann and Starossek (2003) used complex representation to compare the aerodynamic derivatives from the CFD simulations based on the FVM and water tunnel tests.

\subsection{Fully coupled CFD simulations}

Two-dimensional flow simulations on bridge decks for the prediction of coupled flutter limit have been found to give promising agreement with wind tunnel experiments (Frandsen, 2004). These simulations could be used as a supplementary tool to wind tunnel testing for preliminary analysis. Usually, the simulations are performed by free or forced oscillation methods to compute aerodynamic derivatives and then to calculate the flutter limit by using semi-analytical methods in the frequency or time domains. However, these simulations do not represent the actual response of the system at or near the flutter limit because the aerodynamic derivatives are measured at some fixed amplitude with linear assumptions and are sometimes extrapolated, which may lead to erroneous results.

The FSI simulations are time-domain analyses performed using the interaction between flow and a rigid body, as well as its motion. The incompressible $\mathrm{N}-\mathrm{S}$ equations are fully coupled to a rigid body which is elastically suspended on springs with lumped mass. The section of the bridge is considered to move in 3DOF; heave, pitch and lateral motion. Often only the first bending mode and the first torsional mode are considered to contribute to the response for flutter analysis. The instantaneous application of the full wind speed to an initially stationary structure leads to large transient initial motions from which it is difficult to extract definitive conclusions about the stability of small oscillations. This can be avoided by using large fictitious damping only for the first few time-steps and then replacing the actual damping values (Frandsen, 2004).

Computational fluid dynamics has the advantage of simultaneous force measurement and flow visualisation at each point of the domain. Figure 10 shows flow visualisation in a CFD simulation for bluff sections in a uniform flow. Several
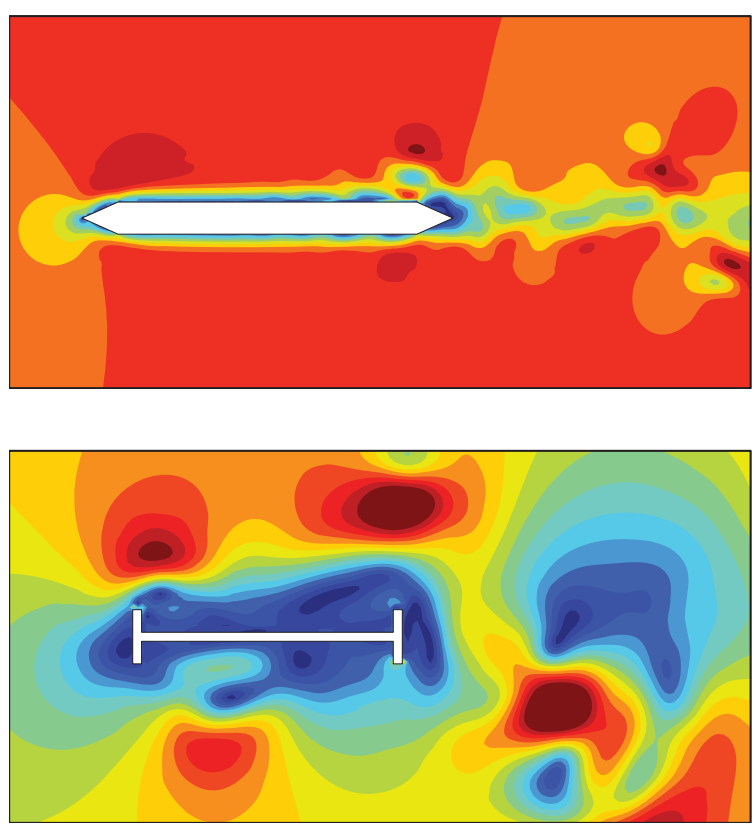

Figure 10. Examples of instantaneous view of velocity fields from CFD simulations for different types of deck sections

studies (Morgenthal and McRobie, 2002; Nowicki and Flaga, 2011; Robertson et al., 2001; Zhan and Fang, 2012) have been performed to show the application and effectiveness of the approach on different test cases. Some other examples of fully coupled CFD simulations to compute flutter limits are given in Tables 4 and 5.

The contribution of higher modes is generally low in longspan bridge flutter, therefore, the 2D approach can provide a reasonable prediction of the flutter limit; however, this contribution can sometimes be significant and cannot be ignored, as discussed in the previous section. The multi-slice or quasi-3D approach (Morgenthal, 2002) can then be used to consider the additional modes where each slice acts as a separate $2 \mathrm{D}$ flow model and the structure is coupled with the help of structural modes. However, this approach has limitations, such as the aerodynamic force correlation is not established, which is insignificant for flutter analysis in a uniform flow.

\subsection{Wind tunnel aeroelastic models}

Generally, there are two types of tests conducted in a wind tunnel for assessing the aeroelastic stability against flutter (Chen and Duan, 2014)

section model tests with spring supports: the aerodynamic characteristics with some selected modes (two or three) can be studied; usually, the model scale is kept larger than $1 / 100$ 
full 3D aeroelastic model: the aerodynamic characteristics of more than three modes can be studied at the same time to examine the coupling effect of different modes.

Some details about the method have been discussed earlier in Section 2.4. Table 6 provides some test examples for long-span bridges. Table 10 shows flutter limits of some well-known bridges around the world.

\section{Effect of parameters and probabilistic approach}

\subsection{Effect of parameters on flutter limit}

\subsubsection{Structural parameters}

The effects of change in the dynamic parameters of a bridge deck model on the prediction of the aerodynamic derivatives are negligible in wind tunnel experiments (Gu et al., 2001). However, uncertainty in the structural parameters can affect the flutter limit prediction significantly, especially the torsional frequency and the mass moment of inertia of the deck (Abbas, 2016; Abbas and Morgenthal, 2014a, 2016). It is also important to consider the frequency ratio between the torsion and the vertical bending. Bartoli et al. (2008) presented the idea to avoid classical flutter by inverting this ratio to have it less than one. Moreover, Zhang and Sun $(2004,2005)$ studied the effect of cable sag, side span length, section depth and dead load on the flutter stability of a suspension bridge.

\subsubsection{Structural damping}

In general, structural damping ratios of up to $2 \%$ have been used in design; however, smaller damping ratios were observed from the outcome of vibration tests of suspension bridges
(Unjoh and Adachi, 1998). The use of structural damping, in flutter analysis, results in a small increase in the flutter limit; however, the variability in structural damping does not have a significant effect on the flutter limit (Khodaparast et al., 2010). Jain et al. (1998) observed that the installation of appropriate external dampers increases the flutter stability of a longspan bridge and can be a viable option to overcome the problems of low flutter speeds.

\subsubsection{Construction stage}

Long-span bridges under erection are more vulnerable to aerodynamic instabilities than in the final state, as the stiffening girder lacks torsional continuity (Zhang, 2004). This makes it important to consider the potential onset of flutter, especially in the early stages of deck erection where, by the use of eccentric ballast, the flutter limit can be enhanced (Larsen, 1997). The sequences of pylons to midspan and unsymmetrical erection can play some favourable role in improving flutter stability (Ge and Tanaka, 2000b; Huang and Li, 2006; Kim et al., 2011). The behaviour of Stonecutters and Sutong cable-stayed bridges against flutter was tested at construction stage by Morgenthal and Yamasaki (2010, 2011). The flutter limit of a bridge under erection may decrease also by the non-linear effects of wind-structure interactions (Zhang, 2004). Jones et al. (2003) studied the effect of mode coupling on flutter during a retrofit and found that flutter can happen on a relatively short-span structure due to the peculiar modal profiles that are created as part of the retrofit procedure.

\subsubsection{Deck shape}

The shape of the deck section greatly influences the flutter stability of long-span bridges. Increasing the aspect ratio $(B / D)$ of

Table 10. Long-span cable-supported bridges with their flutter limits and countermeasures (as of August 2015) (Fujino and Siringoringo, 2013b; Ge and Xiang, 2008a, 2008b; Haifan, 2011; Kreis and André, 2005; Myerscough, 2013; Ostenfeld et al., 1970; Xin and Ou, 2007; Xu, 2013)

\begin{tabular}{lllll}
\hline Bridge name & Main span: $\mathbf{m}$ & Girder type & Flutter limit: $\mathbf{m} / \mathbf{s}$ & Vibration countermeasures \\
\hline $\begin{array}{l}\text { Suspension } \\
\text { Messina }\end{array}$ & 3300 & Triple box & 75 & \\
Akashi-Kaikyo & 1991 & Truss & 84 & Central slot \\
Zhejiang Xihoumen & 1650 & Twin box & 78 & Slotted deck, stabiliser, open grating \\
Great Belt (Storebælt) & 1624 & Single box & 60 & Slotted deck, central slot \\
Little Belt (Lillebælt) & 600 & Single box & 100 & Guide vanes, spoiler \\
Tacoma Narrows & 854 & H-shape & - & Guide vanes \\
Runyang & 1490 & Single box & 75 & Fairings (planned) \\
Tsing Ma & 1377 & Single box & 74 & Centre vertical stabiliser \\
Humen & 888 & Single box & 88 & Central slot \\
Hålogaland & 1145 & Single box & 71 & - \\
Cable-stayed & & & & - \\
Russky & 1104 & Single box & - & - \\
Sutong & 1088 & Single box & 88 & Damper \\
Stonecutters & 1018 & Twin box & 140 & Damper \\
Normandy & 856 & Single box & 78 & -
\end{tabular}


the section, generally, improves the flutter behaviour (Lin et al., 2005). Matsumoto et al. (1996) studied the role of various aspect ratios of a rectangular section on aerodynamic derivatives and flutter stability behaviour. Different configurations of fairings (Fumoto et al., 2005; Miranda and Bartoli, 2001; Permata et al., 2013), fairing angle (Wang et al., 2009), vertical plates (Permata et al., 2013), slot in the middle (Fumoto et al., 2005; Miranda and Bartoli, 2001; Yang et al., 2007, 2009, 2015), double slot and the location of porous cavity/grating and its opening ratios (Matsumoto et al., 2001; Permata et al., 2013) have been studied and proposed to improve the flutter behaviour of the deck section. Abbas (2016) studied the sensitivity of cross-section geometry to the flutter limit for several classes of sections using CFD simulations.

\subsubsection{Atmospheric turbulence}

The effects of turbulence on the FSI have not been fully understood and have been studied only for very limited cases so far (Tamura and Kareem, 2013). Atmospheric turbulence is generally considered beneficial and has been found to raise the flutter limit (Bartoli and Righi, 2006; Haan, 2000; Scanlan, 1997b, 1978b). In other words, free stream turbulence tends to enhance the aerodynamic stability of long-span bridges. Therefore, usually it is ignored in the measurement of aerodynamic derivatives and flutter stability analysis for simplicity (Chen and Duan, 1999; Gimsing and Georgakis, 2011). Parkinson (1989) observed that the increase in large turbulence in wind flow causes a soft galloping section to become weaker and increases stability, and a hard galloping section becomes soft. In some cases the flutter limit is found to increase monotonically with increasing turbulence intensity (Lin et al., 2005). The main effect of an increase in the atmospheric turbulence on a bridge is not to change considerably its drag, lift, moment and aerodynamic derivatives, but to excite several modes of vibration and such response will determine to what extent the bridge is prone to flutter instability. Bucher and Lin (1988), Chen and Kareem (2003a) and $\mathrm{Wu}$ and Kareem (2013) utilised semianalytical models to include the effect of turbulence on flutter. Caracoglia (2009) investigated the implications of the non-linear propagation of an uncertain turbulence field on the $2 \mathrm{D}$ coupled flutter stability of long-span bridges. Turbulent flow can have a negative effect on the flutter stability of long-span bridges with a streamlined section (Huang and Li, 2006). Abbas and Morgenthal (2013b) and Cai and Albrecht (2000) observed that large turbulence intensity may significantly reduce the flutter velocity.

\subsubsection{Non-linear effects}

Geometric and material non-linearities can be considered in the analysis of flutter to investigate post-flutter behaviour by performing non-linear time history analyses and are not usually required for practical applications (Chen and Duan, 1999). However, considering the effect of wind angle on the moment coefficient may result in a significant change in the aeroelastic stability of the bridge (Cheng et al., 2002). Zhang et al. (2002) and Arena and Lacarbonara (2012) considered aerodynamic and structural non-linearities in flutter analysis. The effect of aerodynamic non-linearities in flutter analysis has already been discussed in previous sections.

\subsection{Probabilistic approach to flutter analysis}

The probabilistic assessment of aeroelastic stability against flutter is essential for design consideration of cable-supported long-span bridges (Ge and Xiang, 2008a). The critical design wind speed is determined such that the probability of failure due to flutter is less than or equal to a specified target safety level. Some studies have been conducted on the probabilistic assessment of flutter limit prediction from wind tunnel testing (Ostenfeld-Rosenthar et al., 1992) and CFD applications (Xiang and Ge, 2002).

The Monte-Carlo simulation (MCS) is the traditional and most commonly used method for probabilistic analysis. Usually, the MCS requires a large number of simulations to be performed for different sets of input parameters, which may need significantly large computation time. For this purpose, the response surface method (RSM) can be used to decrease the size of the problem to make this process faster. The RSM represents the structural response by using a simple approximation function. To perform a numerical simulation may take from some minutes to a few days, whereas running this function requires only a fraction of a second. The computation time can be greatly reduced without modifying the existing numerical code for the simulation. These RSMs have been used for probabilistic torsional flutter (Seo and Caracoglia, 2011a, 2011b, 2013) and coupled flutter (Cheng and Xiao, 2005) analyses considering structural parameter uncertainties. Abbas (2016) developed a framework for probabilistic assessment of the flutter prediction models based on the concepts of total uncertainty, which includes parameter uncertainty as well as model uncertainty. The probabilistic assessment also consists of sensitivity analysis, uncertainty analysis, reliability analysis and design optimisation.

\subsubsection{Sensitivity analysis}

Sensitivity analysis can provide essential information about the dependence of the key chosen design variables on the flutter limit of a bridge. The objective of a sensitivity analysis is to identify important parameters and to quantify their effect on the model output. Jurado and Hernández (2004) and Nieto et al. (2011) carried out an analytical sensitivity analysis of the flutter phenomenon in long-span bridges. Sarkar et al. (2007, 2009) and Caracoglia et al. (2009) studied the sensitivities related to extracting experimental aerodynamic derivatives between different laboratory environments. Abbas (2016) and Abbas and Morgenthal (2014a, 2016) studied the sensitivity of 
structural parameters and aerodynamic derivatives to the flutter limit using CFD simulations.

\subsubsection{Uncertainty analysis}

Commonly a flutter analysis is performed considering complete deterministic structural parameters; this is known as a deterministic analysis. However, there are uncertainties in these design variables, which include geometric properties, material properties, load magnitude and distribution (Cheng et al., 2003), as well as aerodynamic derivatives (Caracoglia, 2008; Mannini and Bartoli, 2013, 2015; Seo, 2013). This makes it necessary to consider these uncertainties in the analysis for a more complete understanding. Jakobsen and Tanaka (2003) discussed uncertainties in the prediction of the aeroelastic flutter response of cable-supported bridges. Argentini et al. (2014) used MCS to considere the effects of structural and aerodynamic uncertainties. Structural mass and stiffness parameters are shown to have a significant influence on the flutter limit, especially torsional frequency and mass moment of inertia (Abbas, 2016; Abbas and Morgenthal, 2014a, 2016).

There have been several studies conducted on flutter stability analysis considering parameter uncertainties (Cheng et al., 2003; Kwon, 2010), as well as model-form and predictive uncertainty (Abbas, 2016; Riley, 2011; Riley and Grandhi, 2010, 2011; Riley et al., 2010), by using MCS and utilising the advantages of the RSM. Additionally, Alduse et al. (2015) proposed a Bayesian approach to determine the fatigue damage using bounds and exceedance probability. Khodaparast et al. (2010) used interval, fuzzy and probabilistic methods to propagate the structural uncertainty through an aeroelastic analysis.

\subsubsection{Reliability analysis}

The lack of complete knowledge and uncertainties in the design variables suggest a probabilistic reliability analysis of a long-span bridge to estimate the probability of failure due to flutter instability for a given return period. The probability of bridge failure due to flutter is determined by establishing the limit state as a function of the resistance variable and the load variable (Ge et al., 2000). Pourzeyanali and Datta (2002) used the basic theory of reliability considering uncertainties in geometric and mechanical properties of the bridge, modelling, damping and experimentally obtained aerodynamic derivatives. Different sampling techniques are used to reduce the computational cost for this purpose, such as the importance sampling updating method (Cheng et al., 2005) and line sampling (Shufang et al., 2009). Some improvements have been suggested in flutter reliability analysis by the use of a quadratically asymptotic integral method (Li et al., 2012) and by using a generalised formulation for random eigenvalue analysis for bridge flutter (Canor et al., 2015).

\subsubsection{Optimisation}

Long-span bridges are very important as well as expensive structures; therefore, it is necessary to safeguard them against aeroelastic instabilities while minimising the cost. Nieto et al. (2008) reviewed the analytical formulation of the optimisation process, whereas Hernández et al. (2012) presented a technique that allows the optimum design of deck shape considering both structural and aeroelastic constraints to identify the optimum bridge deck shape. Kusano et al. (2013, 2014, 2015) used several reliability-based design optimisation methods to minimise the bridge girder weight by varying the thickness of the girder plates while satisfying the structural reliability level under flutter considering uncertainties in extreme wind velocity, as well as in aerodynamic derivatives. Xu (2014) proposed an optimisation model using a diagrammatical technique to determine the critical flutter parameters.

\section{Additional considerations}

\subsection{Usefulness of aerodynamic derivatives}

The aeroelastic instability of a bridge is related to the magnitudes and signs of some of the aerodynamic derivatives of the deck cross-section. Therefore, aerodynamic derivatives can be used for preliminary examination of aeroelastic instability. The terms on the right-hand side of Equations $5 \mathrm{a}-5 \mathrm{c}$ can be separated into aerodynamic damping terms (associated with the velocity of motion) and aerodynamic stiffness terms (associated with the displacement of motion). The effect of the change in the damping related forces is considered to be more important for aeroelastic instability. The aerodynamic derivative related to velocity-proportional forces describes the aerodynamic damping of that DOF and the positive sign of this derivative indicates negative aerodynamic damping. The SDOF mechanism of aerodynamic instability is related to the occurrence of negative aerodynamic damping. The aerodynamic derivative $H_{1}^{*}$, which is the aeroelastic lift force induced as a result of heave velocity, controls the vertical flutter and the aerodynamic derivative $A_{2}^{*}$, which is the aeroelastic moment induced as a result of pitch velocity, governs the torsional flutter instability. The flutter phenomenon is related to negative aerodynamic damping whereas negative aerodynamic stiffness creates divergence.

In the case of a flat plate for SDOF instability, considering pure bending motion (refer to Equation 5a), the negative values of $H_{1}^{*}<0$ and $H_{4}^{*}<0$ will increase the overall system damping and stiffness, respectively. If the magnitude of the negative aerodynamic damping becomes greater than the structural damping, the amplitudes of oscillations will grow and aeroelastic instability will occur, which happens usually for deep sections. Similarly, considering pure torsional motion (refer to Equation $5 \mathrm{~b}$ ), where $A_{2}^{*}<0$ and $A_{3}^{*}>0$ the overall system damping will increase and overall system stiffness will 
Table 11. Types of aerodynamic instabilities (after Holmes (2015) and Gimsing and Georgakis (2011))

\begin{tabular}{llll} 
Name & Condition & Type of motion & Type of section \\
\hline Galloping & $H_{1}^{*}>0$ & Translational & D-shape, square section, deep sections \\
Torsional flutter & $A_{2}^{*}>0$ & Rotational & H-section, rectangle with smaller aspect ratio \\
Classical flutter & $H_{2}^{*}>0, A_{1}^{*}>0$ & Coupled & Flat plate, aerofoils
\end{tabular}

decrease. So it is unlikely that SDOF instability will occur and 2DOF flutter is more likely to happen (Zhan et al., 2013).

Table 11 shows some guidelines for assessment of aeroelastic instability by aerodynamic derivatives of the section. The values $H_{4}^{*}$ and $A_{3}^{*}$ tend to increase vertical stiffness and reduce torsional stiffness. For a streamlined section, $-H_{3}^{*}$ and $A_{4}^{*}$ feed energy into structural vibration, whereas aerodynamic damping terms $H_{1}^{*}$ and $A_{2}^{*}$ extract energy. The phase difference for streamline sections is $180^{\circ}$ and the system is less dependent on the structural damping (Dyrbye and Hansen, 1997). Coupled flutter can still occur even if $A_{2}^{*}$ and $H_{1}^{*}$ are positive. Trein and Shirato (2011) reviewed the roles of aerodynamic derivatives in flutter stabilisation and pointed out some conditions that the unsteady pressure characteristics should present in flutter stabilisation.

If the instability limit exceeds the design wind speed of the site at the deck height (suitably factored for ultimate limit states), then modifications are required to be made to the deck crosssection in order to avoid the occurrence of aeroelastic instability. Section modifications can be made to avoid the positive $\left(H_{1}^{*}, A_{2}^{*}\right)$ aerodynamic derivatives (Chen and Duan, 1999). Matsumoto (2013) studied the stabilization of flutter in a longspan bridge by reduction of $A_{2}^{*}$ through suitably combining the deck section with $\left(A_{1}^{*}>0, A_{2}^{*}<0\right)$ and $\left(A_{1}^{*}<0, A_{2}^{*}>0\right)$.

\subsection{Countermeasures to improve flutter stability}

The design of long-span bridges requires complete elimination of the possibility of aeroelastic instability occurring within the design life. The design should ensure that the critical flutter limit of the bridge under the worst condition is still higher than the reference wind speed. Long-span bridge girders can experience unstable flutter oscillations in very strong winds. The objective of flutter control is to enhance the critical flutter limit of the bridge by introducing some modifications to the structural system. These modifications can be broadly classified into three main types based on their function, as follows

deck shape: changing or modifying deck cross-section shape to change the aerodynamic behaviour, which results in reducing the aeroelastic forces and increasing the flutter limit

- tuned mass damper (TMD): introducing TMD increases the structural damping, which reduces some critical structural mode responses under wind action control flaps: additional wing-like components attached to the section, which often require external power and change their relative position or orientation under structural vibration.

Fujino and Siringoringo (2013a, 2013b) and Fujino et al. (2010, 2012) review long-span bridge vibrations under wind action and also describe some solutions as countermeasures for these vibrations. Figure 11 and Table 12 show some countermeasures to improve the behaviour of sections against flutter. Non-streamlined sections with smaller aspect ratios $(B / D)$ are susceptible to galloping. This can be improved by using aerodynamic countermeasures such as skirts and horizontal plate on the lower side of the cross-section (Fujino and Siringoringo, 2013b); this in turn reduces the wind velocity separated from the lower corner of the leading edge of the crosssection, thus reducing the self-excitation forces. Long and slender bridges with bluff sections of large aspect ratio are prone to torsional or coupled flutter. Low torsional rigidity and aerodynamically unstable girder cross-sections are the main factors responsible for torsional flutter, which caused the dramatic collapse of the original Tacoma Narrows Bridge. This can be avoided by streamlining the girder using a closed box section or by using a truss-stiffened girder with increased torsional rigidity.

Flatter streamline sections are generally prone to coupled flutter, which occurs as a result of large pressure differences between upper and lower surfaces of the deck. This pressure difference can be reduced using an open grating or a central slot to improve behaviour against flutter instability. Several
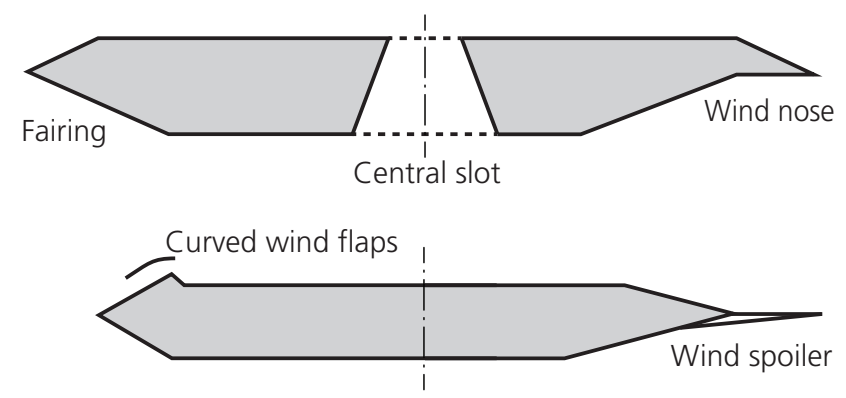

Figure 11. Aerodynamic countermeasure for bridge deck against flutter (after Fujino and Siringoringo (2013a, 2013b)) 
Table 12. Typical aerodynamic countermeasure used for bridge sections against aeroelastic instability phenomena (after Fujino and Siringoringo (2013b))

\begin{tabular}{lll}
\hline Instability phenomenon & Girder type & Countermeasure \\
\hline Galloping & Deep box & Low skirts, horizontal plates \\
Torsional flutter & H-section, rectangle with smaller aspect ratio & Fairings \\
Classical flutter & Deep truss & Open grating, vertical stabiliser \\
& Streamlined & Fairing, central slots, curved wind flaps, wind nose, wind spoilers
\end{tabular}

other attachments can be used to improve flutter stability, such as fairings, grating, wind nose, slots, flaps and spoilers. Torsional flutter stability can be improved by attaching rails to the bottom of the fairing and vertical plates to the lower flange of the girder (Ogawa et al., 2002).

Ge and Xiang (2009a) studied aerodynamic stabilisation for bridges with a central stabiliser and adopting twin-box girders. It was found that the limit of span length for a traditional suspension bridge due to aerodynamic stability can be greatly increased from about $1500 \mathrm{~m}$ up to $5000 \mathrm{~m}$ by using a widely slotted deck or a narrowly slotted deck with vertical and horizontal stabilisers. Sato et al. (2002) confirmed that a slotted box girder was applicable for the super-long-span bridge because it is excellent in terms of economical efficiency and aerodynamic stability.

The use of a TMD on a long-span bridge has been shown to be promising for reduction of wind-induced vibration response. The effectiveness of these TMDs has been extended by changing the design from a single TMD (Chen and Kareem, 2003d) to multiple tuned mass dampers (MTMDs) (Lin et al., 2000) as well as the non-linear TMD (Gattulli et al., 2004). Körlin and Starossek (2004) and Körlin et al. (2012) introduced the rotational mass damper, the movable eccentric mass damper and mass damper system with two eccentric rotational actuators for active bridge deck flutter control. Using the concept of eccentric mass, Phongkumsing et al. (2001) presented a method of suppression of flutter by placing the auxiliary mass on the windward side of a bridge deck to shift the centre of gravity, thus reducing the aerodynamic moment acting on the deck. Aslan and Starossek (2008) presented a passive aeroelastic damper for long-span bridges which consists of a TMD, control surfaces and a transmission, which couples the movement of the TMD with the control surfaces without the need for an external energy supply.

In some cases, dissipative dampers working in the horizontal direction between deck and piers have proved ineffective against flutter. This has been evaluated through a refined model of the controlled bridge with wind interaction forces (Domaneschi and Martinelli, 2014). Devices implemented in the horizontal plane do not influence the torsional stiffness of the bridge deck and therefore the flutter limit is not affected.
Furthermore, the control forces exchanged between the deck and the towers, quite far from the centre span, are not able to improve the flutter stability behaviour.

The aerodynamic-based control method has also proven to be effective in terms of the aerodynamic stability. This method uses control surfaces to generate forces counteracting the unstable oscillations. Graham et al. (2011) used an integrated controllable trailing-edge flap and Huynh and ThoftChristensen (2001) used separate control flaps to show an effective increase in the critical flutter limit of a suspension bridge. Phan and Nguyen (2013) focused on a mechanically controlled system using a passive flap control driven by the motions of the bridge deck. Li et al. (2015) presented an active aerodynamic control method of flutter oscillation comprising a twin-winglet system, which can improve aerodynamic stability to some extent.

\subsection{Beneficial use of flutter}

Flutter is generally considered as a destructive phenomenon, but it can be turned into an environmentally friendly energy source by utilising small plate-like devices as energy harvesters. Energy harvesters are typically small-scale and transform the kinetic energy of wind into electrical energy to provide power to small-scale electronic devices such as wireless sensors and mobile electronic devices (Singh et al., 2012). The most commonly used system is based on the LCO of flexible plate by coupling the aerodynamic and non-linear equations of motion of the plate. Tang et al. (2003) describe an experimental investigation to study flutter and LCO of plates. Peng and Chen (2012) present some background and state-of-the-art developments in this field. Energy harvesting is an active research field and is growing as the demand for renewable energy sources continues to increase (Park et al., 2014). In addition, Matsumoto (2012) studied a mechanism for generating propulsion force for a small ship by flapping a plate.

\section{Concluding remarks on bridge flutter}

The aim of this paper is to provide an overview of existing methods and models used to predict flutter limit by indicating the available literature. Some important conclusions are drawn in the light of this review. Theodorsen's flat plate prediction is simple and easy to use but is only for 2D flow and flat plates or aerofoils, which cannot be used for bridge cross-sections. 
However, it serves as a starting point or reference value to compare actual flutter limits. Scanlan's model is more commonly used as it gives a more direct assessment about the nature of the aerodynamic behaviour of the cross-section.

Frequency domain methods are commonly used because of their simplicity and efficiency; however, they are only for linear structure models and do not consider non-linearities. Therefore, the major part of the current research on the analytical models is to develop the time-domain methods in order to consider aerodynamic non-linearities and fading fluid memory. Simplified approaches are useful to understand the mechanism related to the flutter phenomenon, but cannot be trusted for the final design. CFD is still a developing field and at this point provides a useful alternative to wind tunnel tests only for preliminary design; for final designs, however, wind tunnel tests are preferred.

The choice of bridge deck section is important where the aerodynamic behaviour is governing design considerations. It can save a lot of effort and resources if this is suitably selected in the early design stages. Changing the shape of the deck can increase the flutter limit to a great extent. The MTMD is more desirable than flaps and control systems, which require an external power supply. The probabilistic approaches provide very important insight into the phenomena. The future of aeroelastic analysis for flutter prediction is the use of CFD approaches coupled with semi-analytical methods.

\section{Acknowledgement}

This research is supported by the German Research Foundation (DFG) by way of the Research Training Group 'Evaluation of coupled numerical and experimental partial models in structural engineering (GRK 1462)', which is gratefully acknowledged by the authors.

\section{REFERENCES}

Abbas T (2011) Flutter Analysis of Suspension Bridges using a Two-Stage Numerical Approach and Aerodynamic Derivatives. Masters thesis, Bauhaus-Universität Weimar, Weimar, Germany. Abbas T (2016) Assessment of Numerical Prediction Models for Aeroelastic Instabilities of Bridges. $\mathrm{PhD}$ thesis, Bauhaus-Universität Weimar, Weimar, Germany.

Abbas T and Morgenthal G (2012a) Hybrid models for assessing the flutter stability of suspension bridges. In Proceedings of the 7th International Colloquium on Bluff Body Aerodynamics and Applications (BBAA7), Shanghai, China (Xiang H, Ge Y and Cao S (eds)). China Communications Press, Beijing, China, pp. 490-499.

Abbas T and Morgenthal G (2012b) Model combinations for assessing the flutter stability of suspension bridges. In Proceedings of the 19th International Conference on the Applications of Computer Science and Mathematics in Architecture and Civil Engineering (IKM2012), Weimar, Germany (Gürlebeck K, Lahmer T and
Werner F (eds)). Bauhaus-Universität Weimar, Weimar, Germany, pp. $1-11$.

Abbas T and Morgenthal G (2013a) Quantification of the effects of turbulence in wind on the aeroelastic behaviour of suspension bridges. In Der 13, Dreiländertagung der Windtechnologischen Gesellschaft (WtG2013), Vienna, Austria.

Abbas T and Morgenthal G (2013b) Quantification of the effects of turbulence in wind on the flutter stability of suspension bridges In Proceedings of the 6th European and African Conference on Wind Engineering (EACWE2013), Cambridge, UK, paper no. 71

Abbas T and Morgenthal G (2013c) Quantification of the effects of turbulence in wind on the Vortex-induced vibrations. In Proceedings of the 36th Symposium of the International Association for Bridge and Structural Engineering (IABSE2014), Kolkata, India, paper no. 141 .

Abbas T and Morgenthal G (2014a) Assessment of the flutter stability of bridges using a probabilistic approach. In Proceedings of the 12th International Probabilistic Workshop (IPW12), Weimar, Germany, pp. 17-24.

Abbas T and Morgenthal G (2014b) Numerical models for flutter analysis. In Proceedings of the 6th International Symposium on Computational Wind Engineering (CWE2014), Hamburg, Germany, paper no. 118.

Abbas T and Morgenthal G (2016) Framework for sensitivity and uncertainty quantification in the flutter assessment of bridges. Probabilistic Engineering Mechanics 43: 91-105, https://doi.org/ 10.1016/j.probengmech.2015.12.007.

Agar TJA (1989) Aerodynamic flutter analysis of suspension bridges by a modal technique. Engineering Structures 11(2): 75-82.

Agar TJA (1991) Dynamic instability of suspension bridges. Computers and Structures 41(6): 1321-1328.

Al-Assaf A (2006) Flutter Analysis of Open-Truss Stiffened Suspension Bridges using Synthesized Aerodynamic Derivatives. PhD thesis, Washington State University, Washington, DC, USA.

Alduse BP, Jung S, Vanli OA and Kwon SD (2015) Effect of uncertainties in wind speed and direction on the fatigue damage of long-span bridges. Engineering Structures 100: 468-478, https://doi.org/ 10.1016/j.engstruct.2015.06.031.

Arena A and Lacarbonara W (2012) Nonlinear parametric modeling of suspension bridges under aeroelastic forces: torsional divergence and flutter. Nonlinear Structural Mechanics 70(4): 2487-2510.

Argentini T, Pagani A, Rocchi D and Zasso A (2014) Monte Carlo analysis of total damping and flutter speed of a long span bridge: effects of structural and aerodynamic uncertainties. Journal of Wind Engineering and Industrial Aerodynamics 128: 90-104, https://doi.org/10.1016/j.jweia.2014.02.010.

Aslan H and Starossek U (2008) Passive control of bridge deck flutter using tuned mass dampers and control surfaces. In Proceedings of the 7th European Conference on Structural Dynamics (Eurodyn2008), Southampton, UK (Brennan MJ (ed.)). University of Southampton, Institute of Sound Vibration and Research, Southampton, UK, pp. 7-9.

Astiz M (1998) Flutter stability of very long suspension bridges. Journal of Bridge Engineering 3(3): 132-139.

Bai Y, Sun D and Lin J (2010) Three dimensional numerical simulations of long-span bridge aerodynamics, using block-iterative coupling and DES. Computers \& Fluids 39(9): 1549-1561.

Banerjee J (2003) A simplified method for the free vibration and flutter analysis of bridge decks. Journal of Sound and Vibration 260(5): 829-845.

Bartoli G and Mannini C (2008) A simplified approach to bridge deck flutter. Journal of Wind Engineering and Industrial Aerodynamics 96(2): 229-256. 
Bartoli G and Righi M (2006) Flutter mechanism for rectangular prisms in smooth and turbulent flow. Journal of Wind Engineering and Industrial Aerodynamics 94(5): 275-291.

Bartoli G, D'Asdia P, Febo S et al. (2008) Innovative solutions for long-span suspension bridges. In Proceedings of the 6th International Colloquium on Bluff Body Aerodynamics and Applications (BBAA6), Milan, Italy, paper no. BDG13.

Bartoli G, Contri S, Mannini C and Righi M (2009) Toward an improvement in the identification of bridge deck flutter derivatives. Journal of Engineering Mechanics 135(8): 771-785.

Beliveau JG, Shinozuka M and Vaicaitis R (1977) Motion of suspension bridge subjected to wind loads. Journal of the Structural Division 103(6): 1189-1205.

Billah KY and Scanlan RH (1991) Resonance, Tacoma Narrows bridge failure, and undergraduate physics textbooks. American Journal of Physics 59(2): 118-124.

Bisplinghoff RL, Ashley H and Halfman RL (1996) Aeroelasticity. Dover Publications, New York, NY, USA.

Bleich F (1948) Dynamic instability of truss-stiffened suspension bridges under wind action. Proceedings of ASCE 78(8): $1269-1314$.

Blevins R and Iwan W (1974) The galloping response of a two-degreeof-freedom system. Applied Mechanics 41(4): 1113-1118.

Blocken B (2014) 50 years of computational wind engineering: past, present and future. Journal of Wind Engineering and Industrial Aerodynamics 129: 69-102, https://doi.org/10.1016/j.jweia.2014. 03.008 .

Boonyapinyo V and Janesupasaeree T (2009) Identification of flutter derivatives of bridge decks in wind tunnel test by stochastic subspace identification. American Jounal of Engineering and Applied Sciences 2(2): 304-316.

Boonyapinyo V and Janesupasaeree T (2010) Data-driven stochastic subspace identification of flutter derivatives of bridge decks. Journal of Wind Engineering and Industrial Aerodynamics 98(12): 784-799.

Boonyapinyo V, Miyata T and Yamada H (1999) Advanced aerodynamic analysis of suspension bridges by state-space approach. Journal of Structural Engineering 125(12): 1357-1366.

Boonyapinyo V, Janesupasaeree T and Thamasungkeeti W (2009) Identification of flutter derivatives of bridge decks by stochastic subspace method. In Proceedings of the 7th Asia-Pacific Conference on Wind Engineering (APCWE7), Taipei, Taiwan, paper no. T1-A2.

Borri C and Costa C (2004) Quasi-steady analysis of a two-dimensional bridge deck element. Computers and Structures 82(13-14): 993-1006.

Borri C and Höffer R (2000) Aeroelastic wind forces on flexible bridge girders. Meccanica 35(1): 1-15.

Borri C and Mannini C (2010) Aeroelastic Phenomena and Pedestrian-structure Dynamic Interaction on Non-conventional Bridges and Footbridges. Firenze University Press, Florence, Italy, Strumenti per la didattica e la ricerca (teaching and research tools).

Borri C, Costa C and Zahlten W (2002) Non-stationary flow forces for numerical simulation of aeroelastic instability of bridge decks. Computers and Structures 80(12): 1071-1079.

Brar P, Raul R and Scanlan RH (1996) Numerical calculation of flutter derivatives via indicial functions. Journal of Fluids and Structures 10(4): $337-351$.

Brownjohn J and Jakobsen JB (2001) Strategies for aeroelastic parameter identification from bridge deck free vibration data. Journal of Wind Engineering and Industrial Aerodynamics 89(13): 1113-1136.
Bruno L and Khris S (2003) The validity of 2D numerical simulations of vortical structures around a bridge deck. Mathematical and Computer Modelling 37(7-8): 795-828.

Brusiani F, Cazzoli G, Miranda S, Ubertini F and Vaona P (2010) Estimation of the flutter derivatives of a long-span bridge using the $k$-omega turbulence model. In Proceedings of the XVIII GIMC Conference, Siracuse, Sicily.

Brusiani F, Miranda S, Patruno L, Ubertini F and Vaona P (2013) On the evaluation of bridge deck flutter derivatives using RANS turbulence models. Journal of Wind Engineering and Industrial Aerodynamics 119: 39-47, https://doi.org/10.1016/j.jweia.2013. 05.002 .

BSI (2005) BS EN 1991-1-4:2005: Eurocode 1: Actions on structures. General actions. Wind actions. BSI, London, UK.

Bucher CG and Lin YK (1988) Stochastic stability of bridges considering coupled modes. Journal of Engineering Mechanics 114(12): 2055-2071.

Bucher CG and Lin YK (1989) Stochastic stability of bridges considering coupled modes: II. Journal of Engineering Mechanics 115(2): $384-400$.

Cai CS and Albrecht P (2000) Flutter derivatives based random parametric excitation aerodynamic analysis. Computers and Structures 75(5): 463-477.

Canor T, Caracoglia L and Denoël V (2015) Application of random eigenvalue analysis to assess bridge flutter probability. Journal of Wind Engineering and Industrial Aerodynamics 140: 79-86, https://doi.org/10.1016/j.jweia.2015.02.001.

Caracoglia L (2008) Influence of uncertainty in selected aerodynamic and structural parameters on the buffeting response of long-span bridges. Journal of Wind Engineering and Industrial Aerodynamics 96(3): 327-344.

Caracoglia L (2009) Effects of non-linear propagation of random turbulence fields on bridge flutter instability. In Proceedings of the 5th European and African Conference on Wind Engineering (EACWE2009), Florence, Italy (Borri C (ed.)). Firenze University Press, Firenze, Italy, pp. 353-356.

Caracoglia L and Jones NP (2003a) A methodology for the experimental extraction of indicial functions for streamlined and bluff deck sections. Journal of Wind Engineering and Industrial Aerodynamics 91(5): 609-639.

Caracoglia $L$ and Jones NP (2003b) Time domain vs frequency domain characterization of aeroelastic forces for bridge deck sections. Journal of Wind Engineering and Industrial Aerodynamics 91(3): 371-402.

Caracoglia L, Sarkar PP, Haan FL Jr, Sato H and Murakoshi J (2009) Comparative and sensitivity study of flutter derivatives of selected bridge deck sections, part 2: implications on the aerodynamic stability of long-span bridges. Engineering Structures 31(9): 2194-2202.

Chen A, He X and Xiang H (2002) Identification of 18 flutter derivatives of bridge decks. Journal of Wind Engineering and Industrial Aerodynamics 90(12-15): 2007-2022.

Chen CH, Wu JC and Chen JH (2008) Prediction of flutter derivatives by artificial neural networks. Journal of Wind Engineering and Industrial Aerodynamics 96(10-11): 1925-1937.

Chen W and Duan L (1999) Bridge Engineering Handbook. Taylor \& Francis, London, UK.

Chen W and Duan L (2014) Bridge Engineering Handbook, Superstructure Design, 2nd edn. CRC Press, Boca Raton, FL, USA.

Chen X (2007) Improved understanding of bimodal coupled bridge flutter based on closed-form solutions. Journal of Structural Engineering 133(1): 22-31. 
Chen X and Kareem A (2000) Nonlinear response analysis of long-span bridges under turbulent winds. In Proceedings of the 4th International Colloquium on Bluff Body Aerodynamics and Applications (BBAA4), Bochum, Germany.

Chen X and Kareem A (2001a) Aeroelastic analysis of bridges under multicorrelated winds: integrated state-space approach. Journal of Engineering Mechanics 127(11): 1124-1134.

Chen X and Kareem A (2001b) Nonlinear response analysis of longspan bridges under turbulent winds. Journal of Wind Engineering and Industrial Aerodynamics 89(14-15): 1335-1350.

Chen X and Kareem A (2002) Advances in modeling of aerodynamic forces on bridge decks. Journal of Engineering Mechanics 128(11): 1193-1205.

Chen X and Kareem A (2003a) Aeroelastic analysis of bridges: effects of turbulence and aerodynamic nonlinearities. Journal of Engineering Mechanics 129(8): 885-895.

Chen X and Kareem A (2003b) Anatomy of bridge flutter: some new insights. In Proceedings of the 11th International Conference on Wind Engineering (ICWE11), Lubbock, TX, USA (Smith DA (ed.)).

Chen X and Kareem A (2003c) Curve veering of eigenvalue loci of bridges with aeroelastic effects. Journal of Engineering Mechanics 129(2): 146-159.

Chen X and Kareem A (2003d) Efficacy of tuned mass dampers for bridge flutter control. Journal of Structural Engineering 129(10): 1291-1300.

Chen X and Kareem A (2004) Efficacy of the implied approximation in the identification of flutter derivatives. Journal of Structural Engineering 130(12): 2070-2074.

Chen X and Kareem A (2006a) Revisiting multimode coupled bridge flutter: some new insights. Journal of Engineering Mechanics ASCE 132(10): 1115-1123.

Chen X and Kareem A (2006b) Understanding the underlying physics of multimode coupled bridge flutter based on closed-form solutions. In Proceedings of the 4th International Symposium on Computational Wind Engineering (CWE2006), Yokohama, Japan, pp. $145-148$

Chen X and Kareem A (2008) Identification of critical structural modes and flutter derivatives for predicting coupled bridge flutter. Journal of Wind Engineering and Industrial Aerodynamics 96(10-11): 1856-1870.

Chen X, Matsumoto M and Kareem A (2000a) Aerodynamic coupling effects on flutter and buffeting of bridges. Journal of Engineering Mechanics 126(1): 7-16.

Chen X, Matsumoto M and Kareem A (2000b) Time domain flutter and buffeting response analysis of bridges. Journal of Engineering Mechanics 126(1): 17-26.

Chen X, Kareem A and Matsumoto M (2001) Multimode coupled flutter and buffeting analysis of long span bridges. Journal of Wind Engineering and Industrial Aerodynamics 89(7-8): 649-664.

Cheng J and Xiao R (2005) Probabilistic free vibration and flutter analyses of suspension bridges. Engineering Structures 27(10): $1509-1518$.

Cheng J, Jiang JJ, Xiao RC and Xiang HF (2002) Nonlinear aerostatic stability analysis of Jiang Yin suspension bridge. Engineering Structures 24(6): 773-781.

Cheng J, Jiang JJ and Xiao RC (2003) Aerostatic stability analysis of suspension bridges under parametric uncertainty. Engineering Structures 25(13): 1675-1684.

Cheng J, Cai CS, Xiao RC and Che SR (2005) Flutter reliability analysis of suspension bridges. Journal of Wind Engineering and Industrial Aerodynamics 93(10): 757-775.
Chowdhury AG and Sarkar PP (2003) A new technique for identification of eighteen flutter derivatives using a three-degree-of-freedom section model. Engineering Structures 25(14): 1763-1772.

Chowdhury AG and Sarkar PP (2005) Experimental identification of rational function coefficients for time-domain flutter analysis. Engineering Structures 27(9): 1349-1364.

Costa C (2007) Aerodynamic admittance functions and buffeting forces for bridges via indicial functions. Journal of Wind Engineering and Industrial Aerodynamics 23(3): 413-428.

Costa C and Borri C (2006) Application of indicial functions in bridge deck aeroelasticity. Journal of Wind Engineering and Industrial Aerodynamics 94(11): 859-881.

Daito Y, Matsumoto M and Araki K (2002) Torsional flutter mechanism of two-edge girders for long-span cable-stayed bridge. Journal of Wind Engineering and Industrial Aerodynamics 90(12-15): 2127-2141.

D'Asdia P and Sepe V (1998) Aeroelastic instability of long-span suspended bridges: a multi-mode approach. Journal of Wind Engineering and Industrial Aerodynamics 74-76: 849-857, https://doi.org/10.1016/S0167-6105(98)00077-4.

Davenport A and King J (1984) Dynamic wind forces on long span bridges. In IABSE Congress Report. International Association for Bridge and Structural Engineering (IABSE), ETH-Zurich, Zurich, Switzerland.

Diana G, Cheli F, Zasso A, Collina A and Brownjohn J (1992) Suspension bridge parameter identification in full scale test. Journal of Wing Engineering and Industrial Aerodynamics 41(1-3): 165-176.

Diana G, Bruni S, Cigada A and Collina A (1993) Turbulence effect on flutter velocity in long span suspension bridges. Journal of Wing Engineering and Industrial Aerodynamics 48(2-3): 329-342.

Diana G, Falco M, Bruni S et al. (1995) Comparisons between wind tunnel tests on a full aeroelastic model for proposed bridge over Stretto di Messina and numerical results. Journal of Wing Engineering and Industrial Aerodynamics 54-55: 101-113, https://doi.org/10.1016/0167-6105(94)00034-B.

Diana G, Bruni S, Cigada A and Zappa E (2002) Complex aerodynamic admittance function role in buffeting response of a bridge deck. Journal of Wing Engineering and Industrial Aerodynamics 90(12-15): 2057-2072.

Diana G, Resta F and Rocchi D (2008) A new numerical approach to reproduce bridge aerodynamic non-linearities in the time domain. Journal of Wing Engineering and Industrial Aerodynamics 96(10-11): 1871-1884.

Diana G, Rocchi D, Argentini T and Muggiasca S (2010) Aerodynamic instability of a bridge deck section model: linear and nonlinear approach to force modeling. Journal of Wing Engineering and Industrial Aerodynamics 98(6-7): 363-374.

Diana G, Rocchi D and Argentini T (2013) An experimental validation of band superposition model of the aerodynamic forces acting on a multi-box deck sections. Journal of Wing Engineering and Industrial Aerodynamics 113: 40-58, https://doi.org/10.1016/ j.jweia.2012.12.005.

Ding Q, Chen A and Xiang H (2002) Coupled flutter analysis of long-span bridges by multimode and full-order approaches. Journal of Wind Engineering and Industrial Aerodynamics 90(12-15): 1981-1993.

Diogo G, Lopes AV and Simões LMC (2005) A CFD based numerical study on aerodynamic characteristics of pi cross sections using baffle plates. Proceedings of the 3rd International Conference on Fluid Structure Interaction, Corunha, Spain, vol. 84, pp. 35-44.

Domaneschi M and Martinelli L (2014) Refined optimal passive control of buffeting-induced wind loading of a suspension bridge. Wind and Structures 18(1): 1-20. 
Dowell EH (2004) A Modern Course in Aeroelasticity. Springer, Dordrecht, the Netherlands.

Duncan WJ and Frazer RA (1928) A Brief Survey of Wing Flutter with an Abstract of Design Recommendations. Aeronautical Research Commission R. \& M. 1177, Technical report.

Dung NN, Miyata T, Yamada H and Minh NN (1998) Flutter responses in long span bridges with wind induced displacement by the mode tracing method. Journal of Wind Engineering and Industrial Aerodynamics 77-78: 367-379, https://doi.org/10.1016/ S0167-6105(98)00157-3.

Dyrbye C and Hansen S (1997) Wind Loads on Structures. Wiley, London, UK.

Falco M, Curami A and Zasso A (1992) Nonlinear effects in sectional model aeroelastic parameters identification. Journal of Wind Engineering and Industrial Aerodynamics 41(1-3): 1321-1332.

Farsani HY, Valentine DT, Arena A, Lacarbonara W and Marzocca P (2014) Indicial functions in the aeroelasticity of bridge decks. Journal of Fluids and Structures 48: 203-215, https://doi.org/10.1016/ j.jfluidstructs.2014.02.015.

Frandsen A (1966) Wind stability of suspension bridges. Application of the theory of thin airfoils. Proceedings of the International Symposium on Suspension Bridges, Lisbon, Portugal, pp. 609-627.

Frandsen J (2001) Simultaneous pressures and accelerations measured full-scale on the Great Belt East suspension bridge. Journal of Wind Engineering and Industrial Aerodynamics 89(1): 95-129.

Frandsen J (2004) Numerical bridge deck studies using finite elements. Part I: flutter. Journal of Wind Engineering and Industrial Aerodynamics 19(2): 171-191.

Fu C and Wang S (2014) Computational Analysis and Design of Bridge Structures. CRC Press, Boca Raton, FL, USA.

Fujino $\mathrm{Y}$ and Siringoringo DM (2013a) Long-span bridges vibration, control, seismic retrofit and monitoring - recent studies and lessons learned. In Proceedings of the 6th Civil Engineering Conference in Asia Region (CECAR6), Jakarta, Indonesia, pp. 3-15.

Fujino $Y$ and Siringoringo DM (2013b) Vibration mechanisms and controls of long-span bridges: a review. Structural Engineering International 23(3): 248-268.

Fujino Y, Siringoringo D, Nagayama T and Su D (2010) Control, simulation and monitoring of bridge vibration - Japan's recent development and practice. In Proceedings of the IABSE-JSCE Joint Conference on Advances in Bridge Engineering-II, Dhaka, Bangladesh, pp. 61-74.

Fujino Y, Kimura K and Tanaka H (2012) Wind Resistant Design of Bridges in Japan: Developments and Practices. Springer, Tokyo, Japan.

Fumoto K, Hata K, Matsuda K et al. (2005) Aerodynamic improvement of slotted one-box girder section for super long suspension bridge. In Proceedings of the 6th Asia-Pacific Conference on Wind Engineering (APCWE6), Seoul, South Korea (Choi CK (ed.)), pp. $1222-1236$.

Fung Y (2002) An Introduction to the Theory of Aeroelasticity. Dover Publications, New York, NY, USA

Garrick IE (1938) On Some Reciprocal Relations in the Theory of Nonstationary Flows. National Advisory Committee for Aeronautics (NACA), Langley Field, VA, USA, Report 629.

Gattulli V, Fabio FD and Luongo A (2004) Nonlinear tuned mass damper for self-excited oscillations. Wind and Structures 7(4): 251-264.

Ge Y and Tanaka H (2000a) Aerodynamic flutter analysis of cable-supported bridges by multi-mode and full-mode approaches. Journal of Wind Engineering and Industrial Aerodynamics 86(2-3): $123-153$.
Ge Y and Tanaka H (2000b) Aerodynamic stability of long-span suspension bridges under erection. Journal of Structural Engineering 126(12): 1404-1412.

Ge YJ and Xiang HF (2007) Great demand and great challenge: Chinese major bridges under construction for improving traffic infrastructure nationwide. Proceedings of the Symposium of the International Association for Bridge and Structural Engineering (IABSE2007), Weimar, Germany, pp. 6-9.

Ge $Y$ and Xiang H (2008a) Recent development of bridge aerodynamics in China. Journal of Wind Engineering and Industrial Aerodynamics 96(6-7): 736-768.

Ge YJ and Xiang HF (2008b) Bluff body aerodynamics application in challenging bridge span length. In Proceedings of the 6th International Colloquium on Bluff Body Aerodynamics and Applications (BBAA6), Milan, Italy, paper no. BDG06.

Ge YJ and Xiang HF (2008c) Computational models and methods for aerodynamic flutter of long-span bridges. Journal of Wind Engineering and Industrial Aerodynamics 96(10-11): 1912-1924.

Ge $Y$ and Xiang H (2009a) Aerodynamic stabilization for box-girder suspension bridges with super-long span. In Proceedings of the 5th European and African Conference on Wind Engineering (EACWE2009), Florence, Italy (Borri C (ed.)). Firenze University Press, Firenze, Italy, paper no. 172.

Ge $Y$ and Xiang H (2009b) Theoretical model and method for self-excited aerodynamic forces of long-span bridges. In Proceedings of the International Symposium on Computational Structural Engineering, Shanghai, China (Yuan Y, Cui JZ and Mang H (eds)), pp. 93-99.

Ge YJ, Xiang HF and Tanaka H (2000) Application of a reliability analysis model to bridge flutter under extreme winds. Journal of Wind Engineering and Industrial Aerodynamics 86(2-3): 155-167.

Ge Y, Lin Z, Cao F, Pang J and Xiang H (2002) Investigation and prevention of deck galloping oscillation with computational and experimental techniques. Journal of Wind Engineering and Industrial Aerodynamics 90(12-15): 2087-2098.

Gimsing NJ and Georgakis CT (2011) Cable Supported Bridges: Concept and Design, 3rd edn. John Wiley \& Sons, Ltd, Chichester, UK.

Graham J, Limebeer D and Zhao X (2011) Aeroelastic modelling of long-span suspension bridges. Proceedings of the 18th World Congress of the International Federation of Automatic Control (IFAC2011), Milan, Italy, vol. 18, pp. 9212-9217.

Gu M and Qin XR (2004) Direct identification of flutter derivatives and aerodynamic admittances of bridge decks. Engineering Structures 26(14): 2161-2172.

Gu M, Xiang H and Lin Z (1999) Flutter- and buffeting-based selection for long-span bridges. Journal of Wind Engineering and Industrial Aerodynamics 80(3): 373-382.

Gu M, Zhang R and Xiang H (2000) Identification of flutter derivatives of bridge decks. Journal of Wind Engineering and Industrial Aerodynamics 84(2): 151-162.

Gu M, Zhang R and Xiang H (2001) Parametric study on flutter derivatives of bridge decks. Engineering Structures 23(12): $1607-1613$.

Guo Z and Ge Y (2012) A new state-space model for self-excited forces and straight forward analysis of bridge deck flutter. Proceedings of the 7th International Colloquium on Bluff Body Aerodynamics and Applications (BBAA7), Shanghai, China (Xiang H, Ge Y and Cao S (eds)). China Communications Press, Beijing, China, pp. $480-489$.

Haan FL (2000) The Effects of Turbulence on the Aerodynamics of Long-span Bridges. PhD thesis, University of Notre Dame, Notre Dame, IN, USA. 
Haifan X (2011) Conceptual Design of Bridges. China Communications Press, Beijing, China.

Haung $\mathrm{L}$ and Liao $\mathrm{H}$ (2011) Identification of flutter derivatives of bridge deck under multi-frequency vibration. Engineering Applications of Computational Fluid Mechanics 5(1): 16-25.

Hernández S, Jurado JA, Nieto F and Mosquera A (2008)

Multidisciplinary approach to aeroelastic studies of long-span bridges. Structural and Multidisciplinary Optimization 35(4): 365-374.

Hernández S, Nieto F, Jurado JA and Pérez I (2012) Bluff body aerodynamics of simplified bridge decks for aeroelastic optimization. In Proceedings of the 7th International Colloquium on Bluff Body Aerodynamics and Applications (BBAA7), Shanghai, China (Xiang H, Ge Y and Cao S (eds)). China Communications Press, Beijing, China, pp. 112-121.

Hoa LT (2006) Aerodynamics, stability and response prediction of bridges due to turbulent atmospheric flow. In Proceedings of the National Conference on Aero-Fluid Mechanics, VungTau, Vietnam, paper no. 10.

Hoa LT (2008) Flutter instability of cable-stayed bridges. In Proceedings of the National Scientific Seminar on Vibration of Cable-stayed Bridges, Hanoi, Vietnam.

Holmes J (2015) Wind Loading of Structures, 3rd edn. CRC Press, Boca Raton, FL, USA.

Hua $X$ and Chen Z (2008) Full-order and multimode flutter analysis using ANSYS. Finite Elements in Analysis and Design 44(9-10): 537-551.

Huang $\mathrm{H}$ and Li M (2006) Flutter and buffeting analysis in time domain for suspension bridge in operation and erection stage. In Proceedings of the 4th International Symposium on Computational Wind Engineering (CWE2006), Yokohama, Japan, paper no. TD4-02, pp. 781-784

Huang L, Liao H, Wang B and Li Y (2009) Numerical simulation for aerodynamic derivatives of bridge deck. Simulation Modelling Practice and Theory 17(4): 719-729.

Huang MH, Lin YY and Weng MX (2012) Flutter and buffeting analysis of bridges subjected to skew wind. Journal of Applied Science and Engineering 15(4): 401-413.

Hunyadi M (2009) Flutter analysis of an extradosed bridge in Hungary. In Proceedings of the 5th European and African Conference on Wind Engineering (EACWE2009), Florence, Italy, paper no. 151

Huynh T and Thoft-Christensen P (2001) Suspension bridge flutter for girders with separate control flaps. Journal of Bridge Engineering 6(3): $168-175$

Iwamoto M and Fujino Y (1995) Identification of flutter derivatives of bridge deck from free vibration data. Journal of Wind Engineering and Industrial Aerodynamics 54-55: 55-63, https://doi.org/ 10.1016/0167-6105(94)00029-D.

Jain A, Jones NP and Scanlan RH (1996a) Coupled aeroelastic and aerodynamic response analysis of long-span bridges. Journal of Wind Engineering and Industrial Aerodynamics 60: 69-80, https://doi.org/10.1016/0167-6105(96)00024-4.

Jain A, Jones NP and Scanlan RH (1996b) Coupled flutter and buffeting analysis of long-span bridges. Structural Engineering 122(7): 716-725.

Jain A, Jones NP and Scanlan RH (1998) Effect of modal damping on bridge aeroelasticity. Journal of Wind Engineering and Industrial Aerodynamics 77-78: 421-430, https://doi.org/10.1016/S0167-6105 (98)00161-5.

Jakobsen J and Tanaka H (2003) Modelling uncertainties in prediction of aeroelastic bridge behaviour. Journal of Wind Engineering and Industrial Aerodynamics 91(12-15): 1485-1498.
Janjic D, Stampler J and Domaingo A (2008) Software tool for wind design of cable stayed bridges. In Proceedings of the Structures Congress 2008: crossing boarders, Vancouver, BC, Canada, pp. $1-10$.

Jensen AG (1997) Aeroelastic wind forces on flexible bridge girders. Journal of Wind Engineering and Industrial Aerodynamics 69-71(1): 777-793.

Jones NP and Ozkan E (2002) Wind effects on long span cable stayed bridges: assessment and validation. In Proceedings of the Joint Meeting of USIJapan Natural Resources Development Program (UJNR), Gaithersburg, MD, USA.

Jones NP, Raggett JD and Ozkan E (2003) Prediction of cable-supported bridge response to wind: coupled flutter assessment during retrofit. Journal of Wind Engineering and Industrial Aerodynamics 91(12-15): 1445-1464.

Jones RT (1940) The Unsteady Lift of a Wing of a Finite Aspect Ratio. National Advisory Committee for Aeronautics (NACA), Langley Field, VA, USA, Report 681.

Jurado JA and Hernández S (2000) Theories of aerodynamic forces on decks of long span bridges. Journal of Bridge Engineering 5(1): 813.

Jurado J and Hernández S (2004) Sensitivity analysis of bridge flutter with respect to mechanical parameters of the deck. Structural and Multidisciplinary Optimization 27(4): 272-283.

Jurado J, Hernández S, Nieto F and Mosquera A (2011) Bridge Aeroelasticity: Sensitivity Analysis and Optimal Design (Malhotra VM (ed.)). WIT Press, Southampton, UK, Series on High Performance Structures and Materials. Jurado JA, León A, Nieto F and Hernández S (2008) Experimental and computational enhancement for hybrid analysis of suspension bridges. In Proceedings of the 6th International Colloquium on Bluff Body Aerodynamics and Applications (BBAA6), Milan, Italy, paper no. BDG11.

Jurado JA, Kusano I, Hernández S and Nieto F (2013) Improvement of multimodal flutter analysis code, FLAS. In Proceedings of the 6th European and African Conference on Wind Engineering (EACWE2013), Cambridge, UK.

Kalesar HE and Ali FS (2008) Aeroelastic analysis of suspension bridge decks using finite element method. In Proceedings of the 6th International Colloquium on Bluff Body Aerodynamics and Applications (BBAA6), Milan, Italy, paper no. BDG05.

Karpel M (1981) Design for Active and Passive Flutter Suspension and Gust Alleviation. National Aeronautics and Space Administration (NASA), Washington, DC, USA, Report CR-3482.

Katsuchi H, Jones NP, Scanlan RH and Akiyama H (1998) Multi-mode flutter and buffeting analysis of the Akashi-Kaikyo bridge. Journal of Wind Engineering and Industrial Aerodynamics 77-78: 431-441, https://doi.org/10.1016/S01676105(98)00162-7.

Katsuchi H, Jones NP and Scanlan RH (1999) Multimode coupled flutter and buffeting analysis of the Akashi-Kaikyo bridge. Structural Engineering 125(1): 60-70.

Ketong $L$ and Aiping $T$ (2014) Numerical investigation for aerodynamic derivatives of bridge deck using DES. The Open Civil Engineering Journal 8(1): 326-334.

Khodaparast HH, Mottershead JE and Badcock KJ (2010) Propagation of structural uncertainty to linear aeroelastic stability. Computers and Structures 88(3-4): 223-236.

Kim YM, Kwak YH, Choi MS, Lee JJ and Cho KS (2011) Wind engineering on the New Millennium Bridge in South Korea. Procedia Engineering 14: 1472-1479, https://doi.org/10.1016/j.proeng. 2011.07.185. 
Klöppel K and Thiele F (1967) Modellversuche im windkanal zur bemessung von brücken gegen die gefahr winderregter schwingungen. Der Stahlbau 36(12): 353-365 (in German).

Körlin R and Starossek U (2004) Active mass dampers for flutter control of bridges. In Proceedings of the 8th International Conference on Flow-Induced Vibrations (FIV2004), Paris, France.

Centre Poly-Media, Palaiseau, France.

Körlin R, Boonto S, Werner H and Starossek U (2012) LMI-based gain scheduling for bridge flutter control using eccentric rotational actuators. Optimal Control Applications and Methods 33(4): 488-500.

Kovacs I, Svensson HS and Jordet E (1992) Analytical aerodynamic investigation of cable-stayed Helgeland Bridge. Journal of Structural Engineering 118(1): 147-168.

Kreis E and André J (2005) A numerical inquiry into the flutter phenomenon in long-span bridges. Latin American Journal of Solids and Structures 2(4): 321-337.

Kurniawan R (2013) Numerical study of flutter of a two-dimensional aeroelastic system. In Proceedings of the World Congress on Engineering (WCE2013), London, UK, vol. III, pp. 1858-1860.

Kusano I, Baldomir A, Ángel Jurado J and Hernández S (2013) Reliability based design optimization of long-span bridges considering flutter. In Proceedings of the 6th European and African Conference on Wind Engineering (EACWE2013), Cambridge, UK, paper no. 10.

Kusano I, Baldomir A, Ángel Jurado J and Hernández S (2014) Reliability based design optimization of long-span bridges considering flutter. Journal of Wind Engineering and Industrial Aerodynamics 135: 149-162, https://doi.org/10.1016/j.jweia.2014.10.006.

Kusano I, Baldomir A, Ángel Jurado J and Hernández S (2015) Probabilistic optimization of the main cable and bridge deck of long-span suspension bridges under flutter constraint. Journal of Wind Engineering and Industrial Aerodynamics 146: 59-70, https:// doi.org/10.1016/j.jweia.2015.08.001.

Kvamstad TH (2011) Assessment of the Flutter Stability Limit of the Hålogaland Bridge using a Probabilistic Approach. Masters thesis, Department of Structural Engineering, Norwegian University of Science and Technology, Trondheim, Norway.

Kwon SD (2010) Uncertainty of bridge flutter velocity measured at wind tunnel tests. In Proceedings of the 4th International Symposium on Computational Wind Engineering (CWE2010), Chapel Hill, NC, USA, paper no. 293.

Larsen A (1993) Aerodynamic aspects of the final design of the $1624 \mathrm{~m}$ suspension bridge across the Great Belt. Journal of Wind Engineering and Industrial Aerodynamics 48(2-3) 261-285.

Larsen A (1997) Prediction of aeroelastic stability of suspension bridges during erection. Journal of Wind Engineering and Industrial Aerodynamics 72: 265-274, https://doi.org/10.1016/S0167-6105(97) 00248-1.

Larsen A (1998) Advances in aeroelastic analyses of suspension and cable-stayed bridges. Journal of Wind Engineering and Industrial Aerodynamics 74-76: 73-90, https://doi.org/10.1016/S0167-6105 (98)00007-5.

Larsen A (2006) Computation of flutter of long span cable supported bridges. In Proceedings of the 4th International Symposium on Computational Wind Engineering (CWE2006), Yokohama, Japan, pp. $149-152$.

Larsen A and Walther JH (1996) A new computational method for assessment of the aeroelastic stability of long span bridges. In IABSE Congress Report. International Association for Bridge and Structural Engineering (IABSE), ETH-Zurich, Zurich, Switzerland, vol. 15, pp. 93-98.
Larsen A and Walther JH (1997a) Aeroelastic analysis of bridge girder section based on discrete vortex simulations. Journal of Wind Engineering and Industrial Aerodynamics 67-68: 253-265, https://doi.org/10.1016/S0167-6105(97)00077-9.

Larsen A and Walther JH (1997b) A two dimensional discrete vortex method for bridge aerodynamics applications. Journal of Wind Engineering and Industrial Aerodynamics 67-68: 183-193, https://doi.org/10.1016/S0167-6105(97)00072-X.

Larsen A and Walther JH (1998) Discrete vortex simulation of flow around five generic bridge deck sections. Journal of Wind Engineering and Industrial Aerodynamics 77-78: 591-602, https://doi.org/10.1016/S0167-6105(98)00175-5.

Larsen A and Walther J (2003) Discrete vortex simulation of vortex excitation and mitigation in bridge engineering. In Proceedings of the 2nd MIT Conference on Computational Fluid and Solid Mechanics, Cambridge, USA, pp. 1397-1400.

Larsen S, Sinding P and Smitt L (2011) Extraction of aerodynamic flutter derivatives in newly developed forced motion rig with 3 degrees of freedom. In Proceedings of the 13th International Conference on Wind Engineering (ICWE13), Amsterdam, the Netherlands.

Lazzari M (2005) Time domain modeling of aeroelastic bridge decks: a comparative study and an application. International Journal for Numerical Methods in Engineering 62(8): 1064-1104.

Lazzari M, Vitalini RV and Saetta AV (2004) Aeroelastic forces and dynamic response of long-span bridges. International Journal for Numerical Methods in Engineering 60(6): 1011-1048.

Lee HE, Vu TV, Yoo SY and Lee HY (2011) A simplified evaluation in critical frequency and wind speed to bridge deck flutter. Procedia Engineering 14: 1784-1790, https://doi.org/10.1016/j.proeng. 2011.07.224.

Li K, Ge Y, Guo Z and Zhao L (2015) Theoretical framework of feedback aerodynamic control of flutter oscillation for long-span suspension bridges by the twin-winglet system. Journal of Wind Engineering and Industrial Aerodynamics 145: 166-177, https://doi.org/10.1016/j.jweia.2015.06.012.

Li L, Ge Y and Dai G (2012) Probability evaluation of flutter failure for long-span bridges. In Proceedings of the 7th International Colloquium on Bluff Body Aerodynamics and Applications (BBAA7), Shanghai, China (Xiang H, Ge Y and Cao S (eds)). China Communications Press, Beijing, China, pp. 1359-1364.

Li Y, Liao H and Qiang S (2003) Weighting ensemble least-square method for flutter derivatives of bridge decks. Journal of Wind Engineering and Industrial Aerodynamics 91(6): 713-721.

Liao H, Suo Q and Wang Q (2012) Study on the empirical mathematical model of motion-induced aerodynamic force of bridge girders. In Proceedings of the 7th International Colloquium on Bluff Body Aerodynamics and Applications (BBAA7), Shanghai, China (Xiang H, Ge Y and Cao S (eds)). China Communications Press, Beijing, China, pp. 419-428.

Lin YK and Ariaratnam ST (1980) Stability of bridge motion in turbulent winds. Journal of Structural Mechanics 8(1): 1-15.

Lin YK and Li QC (1993) New stochastic theory for bridge stability in turbulent flow. Journal of Structural Mechanics 119(1): 113-127.

Lin YK and Yang JN (1983) Multimode bridge response to wind excitations. Journal of Engineering Mechanics 109(2): 586-603.

Lin YY, Cheng CM and Sun D (2000) Wind-induced vibration control of long-span bridges by multiple tuned mass dampers. Tamkang Journal of Science and Engineering 3(1): 1-13.

Lin YY, Cheng CM, Wu JC, Lan TL and Wu KT (2005) Effects of deck shape and oncoming turbulence on bridge aerodynamics. Tamkang Journal of Science and Engineering 8(1): 43-56. 
Liu S and Ge YJ (2012) A new unsteady aerodynamic model with unique parameters. Proceedings of the 7th International Colloquium on Bluff Body Aerodynamics and Applications (BBAA7), Shanghai, China (Xiang H, Ge Y and Cao S (eds)). China Communications Press, Beijing, China, pp. 441-452.

Liua X, Chen Z and Liu Z (2012) Direct simulation method for flutter stability of bridge deck. In Proceedings of the 7th International Colloquium on Bluff Body Aerodynamics and Applications (BBAA7), Shanghai, China (Xiang $\mathrm{H}, \mathrm{Ge} \mathrm{Y}$ and Cao S (eds)). China Communications Press, Beijing, China, pp. 1528-1535.

Lute V, Upadhyay A and Singh K (2009) Support vector machine based aerodynamic analysis of cable stayed bridges. Advances in Engineering Software 40(9): 830-835.

Maîytre OL, Scanlan R and Knio O (2003) Estimation of the flutter derivatives of an NACA airfoil by means of Navier-Stokes simulation. Journal of Fluids and Structures 17(1): 1-28.

Mannini C (2006) Flutter Vulnerability Assessment of Flexible Bridges. $\mathrm{PhD}$ thesis, University of Florence, Florence, Italy.

Mannini C and Bartoli G (2008) Investigation on the dependence of bridge deck flutter derivatives on steady angle of attack. In Proceedings of the 6th International Colloquium on Bluff Body Aerodynamics and Applications (BBAA6), Milan, Italy, paper no. PST03.

Mannini C and Bartoli G (2013) Uncertainty propagation in bridge flutter analysis. In Proceedings of the 11th International Conference on Structural Safety and Reliability (ICOSSAR2013), New York City, USA, pp. 1719-1726.

Mannini C and Bartoli G (2015) Aerodynamic uncertainty propagation in bridge flutter analysis. Structural Safety 52: 29-39, https://doi.org/10.1016/j.strusafe.2014.07.005.

Mannini C, Bartoli G and Borri C (2012) New developments in bridge flutter analysis. Proceedings of the Institution of Civil Engineers Structures and Buildings 165(3): 139-159.

Matsumoto M (1996) Aerodynamic damping of prisms. Journal of Wind Engineering and Industrial Aerodynamics 59(2-3): 159-175.

Matsumoto M (2009) Vortex effect on torsional flutter. In Proceedings of the 7th Asia-Pacific Conference on Wind Engineering (APCWE7), Taipei, Taiwan, paper no. T1A-1.

Matsumoto M (2012) Flutter and its application - flutter mode and ship navigation. In Proceedings of the 7th International Colloquium on Bluff Body Aerodynamics and Applications (BBAA7), Shanghai, China (Xiang H, Ge Y and Cao S (eds)). China Communications Press, Beijing, China, pp. 23-55.

Matsumoto M (2013) Flutter and its application: flutter mode and ship navigation. Journal of Wind Engineering and Industrial Aerodynamics 122: 10-20, https://doi.org/10.1016/j.jweia.2013. 06.003 .

Matsumoto M, Shiraishi N, Shirato H, Shigetaka K and Niihara Y (1993) Aerodynamic derivatives of coupled/hybrid flutter of fundamental structural sections. Journal of Wind Engineering and Industrial Aerodynamics 49(1-3): 575-584.

Matsumoto M, Nihara Y, Kobayashi Y, Sato H and Hamasaki H (1995) Flutter mechanism and its stabilization of bluff bodies. Proceedings of the 9th International Conference on Wind Engineering (ICWE1995), New Delhi, India, pp. 827-838.

Matsumoto M, Kobayashi Y and Shirato H (1996) The influence of aerodynamic derivatives on flutter. Journal of Wind Engineering and Industrial Aerodynamics 60: 227-239, https://doi.org/10.1016/ 0167-6105(96)00036-0.

Matsumoto M, Daito Y, Yoshizumi F, Ichikawa Y and Yabutani T (1997) Torsional flutter of bluff bodies. Journal of Wind Engineering and Industrial Aerodynamics 69-71: 871-882, https://doi.org/10.1016/ S0167-6105(97)00213-4.
Matsumoto M, Goto K and Yabutani T (1998) Heaving branch coupled flutter for long span bridge. In Proceedings of IABSE Symposium on Long-Span and High-Rise Structures, Kobe, Japan, paper no. 179, pp. 259-264.

Matsumoto M, Yoshizumi F, Yabutani T, Abe K and Nakajima N (1999) Flutter stabilization and heaving-branch flutter. Journal of Wind Engineering and Industrial Aerodynamics 83(1-3): 289-299.

Matsumoto M, Nakajima N, Taniwaki Y and Shijo R (2001) Grating effect on flutter instability. Journal of Wind Engineering and Industrial Aerodynamics 89(14-15): 1487-1497.

Matsumoto M, Taniwaki Y and Shijo R (2002) Frequency characteristics in various flutter instabilities of bridge girders. Journal of Wind Engineering and Industrial Aerodynamics 90(12): 1973-1980.

Matsumoto M, Shirato H, Yagi T et al. (2003) Effects of aerodynamic interferences between heaving and torsional vibration of bridge decks: the case of Tacoma Narrows Bridge. Journal of Wind Engineering and Industrial Aerodynamics 91(12): 1547-1557.

Matsumoto M, Mizuno K, Okubo K and Ito Y (2006a) The complex branch characteristics of coupled flutter. In Proceedings of the 4th International Symposium on Computational Wind Engineering (CWE2006), Yokohama, Japan, pp. 137-140.

Matsumoto M, Okubo K, Ito Y et al. (2006b) Branch switching characteristics of coupled flutter instability. In Proceedings of the 19th KKCNN Symposium on Civil Engineering, Kyoto, Japan.

Matsumoto M, Mizuno K, Okubo K, Ito Y and Matsumiya H (2007) Flutter instability and recent development in stabilization of structures. Journal of Wind Engineering and Industrial Aerodynamics 95(9-11): 888-907.

Matsumoto M, Matsumiya H, Fujiwara S and Ito Y (2008a) New consideration on flutter properties basing on SBS: fundamental flutter mode, similar Selberg's formula, torsional divergence instability, and new coupled flutter phenomena affected by structural coupling. In Proceedings of the 6th International Colloquium on Bluff Body Aerodynamics and Applications (BBAA6), Milan, Italy, paper no. BDG16.

Matsumoto M, Okubo K, Ito Y, Matsumiya H and Kim G (2008b) The complex branch characteristics of coupled flutter. Journal of Wind Engineering and Industrial Aerodynamics 96(10-11): 1843-1855.

Matsumoto M, Shirato H, Mizuno K, Shijo R and Hikida T (2008c) Flutter characteristics of $\mathrm{H}$-shaped cylinders with various side-ratios and comparisons with characteristics of rectangular cylinders. Journal of Wind Engineering and Industrial Aerodynamics 96(6-7): 963-970.

Matsumoto M, Yagi T, Tamaki H and Tsubota T (2008d) Vortex-induced vibration and its effect on torsional flutter instability in the case of $B / D=4$ rectangular cylinder. Journal of Wind Engineering and Industrial Aerodynamics 96(6-7): 971-983.

Matsumoto M, Matsumiya H and Fujiwara S (2009) Branch switch of coupled flutter. In Proceedings of the 7th Asia-Pacific Conference on Wind Engineering ( APCWE7), Taipei, Taiwan, paper no. M1A-1.

Matsumoto M, Matsumiya H, Fujiwara S and Ito $Y$ (2010) New consideration on flutter properties based on step-by-step analysis. Journal of Wind Engineering and Industrial Aerodynamics 98(8-9): 429-437.

McRobie A, Morgenthal G, Abrams D and Prendergast J (2013) Parallels between wind and crowd loading of bridges. Philosophical Transactions of the Royal Society A 371(1993): $1-17$.

Miranda M and Bartoli G (2001) Aerodynamic optimization of decks of cable-stayed bridges. In Proceedings of the Symposium of the International Association for Bridge and Structural Engineering (IABSE 2001), Seoul, South Korea, vol. 84, pp. 34-41. 
Miranda S, Patruno L, Ubertini F and Vairo G (2013) Indicial functions and flutter derivatives: a generalized approach to the motion-related wind loads. Journal of Fluids and Structures 42: 466-487, https://doi.org/10.1016/j.jfluidstructs. 2013.08.009.

Miranda S, Patruno L, Ubertini F and Vairo G (2014) On the identification of flutter derivatives of bridge decks via rans turbulence models: benchmarking on rectangular prisms. Engineering Structures 76: 359-370, https://doi.org/10.1016/ j.engstruct.2014.07.027.

Mishra SS, Kumar K and Krishna P (2008a) Relevance of eighteen flutter derivatives in wind response of a long-span cable-stayed bridge. Journal of Structural Engineering 134(5): 769-781.

Mishra SS, Kumar K and Krishna P (2008b) Multimode flutter of long-span cable-stayed bridge based on 18 experimental aeroelastic derivatives. Journal of Wind Engineering and Industrial Aerodynamics 96(1): 83-102.

Miyata T (2003) Historical view of long-span bridge aerodynamics. Journal of Wind Engineering and Industrial Aerodynamics 91(12): 1393-1410.

Miyata T and Yamaguchi K (1993) Aerodynamics of wind effects on the Akashi Kaikyo bridge. Journal of Wind Engineering and Industrial Aerodynamics 48(2-3): 287-315.

Miyata T, Kubo Y and Ito M (1975) Analysis of aeroelastic oscillations of long-span structures by nonlinear multi-dimensional procedures. In Proceedings of the 4th International Conference on Wind Effects on Buildings and Structures. Cambridge University Press, Cambridge, UK.

Miyata T, Yamada H, Boonyapinyo V and Santos JC (1995a) Analyitical investigation on the response of a very long suspension bridge under gusty wind. Proceedings of the 9th International Conference on Wind Engineering (ICWE1995), New Delhi, India, pp. 1006-1017.

Miyata T, Yamada H, Boonyapinyo V and Santos JC (1995b) Full model testing of large cable-supported bridges. Proceedings of the 9th International Conference on Wind Engineering (ICWE1995), New Delhi, India, pp. 249-280.

Morgenthal G (2000) Comparison of Numerical Methods for Bridge-Deck Aerodynamics. MPhil thesis, University of Cambridge, Cambridge, UK.

Morgenthal G (2002) Aerodynamic Analysis of Structures Using High-Resolution Vortex Particle Methods. PhD thesis, University of Cambridge, Cambridge, UK.

Morgenthal G (2005) Advances in numerical bridge aerodynamics and recent applications. Structural Engineering International (IABSE) 15(2): 95-100.

Morgenthal G (2006) Numerical analysis of bridge aerodynamics. Structural Concrete (fib) 6(1): 35-41.

Morgenthal G and McRobie FA (2002) A comparative study of numerical methods for fluid structure interaction analysis in long-span bridge design. Wind and Structures 5(2): 101-114

Morgenthal G and Walther JH (2007) An immersed interface method for the vortex-in-cell algorithm. Computers and Structures 85(11) $712-726$.

Morgenthal G and Yamasaki Y (2010) Behaviour of very long cable-stayed bridges during erection. Proceedings of Institution of Civil Engineers - Bridge Engineering 163(4): 213-224, http://dx.doi.org/10.1680/bren.2010.4.213.

Morgenthal G and Yamasaki Y (2011) Aerodynamic behaviour of very long cable-stayed bridges during construction. Procedia Engineering 14: 1463-1471, https://doi.org/10.1016/j.proeng.2011. 07.184 .
Morgenthal G, Kovacs I and Saul R (2005) Analysis of aeroelastic bridge deck response to natural wind. Structural Engineering International (IABSE) 15(4): 232-235.

Morgenthal G, Corriols AS and Bendig B (2014) A GPU-accelerated pseudo-3D vortex method for aerodynamic analysis. Journal of Wind Engineering and Industrial Aerodynamics 125: 69-80, https://doi.org/10.1016/j.jweia.2013.12.002.

Myerscough M (2013) Suspension bridges: past and present. The Structural Engineer 91(7): 12-21.

Nakamura Y (1979) On the aerodynamic mechanism of torsional flutter of bluff structures. Journal of Sound and Vibration 67(2): 163-177.

Nakamura Y and Mizota T (1975) Torsional flutter of rectangular prisms. Engineering Mechanics 101(2): 125-142.

Nakamura $\mathrm{H}$ and Ogaki I (1990) Flutter simulation for bridges. In Proceedings of the MSC 1990 World Users Conference, vol. I, pp. 1-13.

Namini A, Albrecht P and Bosch H (1992) Finite element-based flutter analysis of cable-suspended bridges. Journal of Structural Engineering 118(6): 1509-1526.

Neuhaus C, Roesler S, Höffer R and Hortmanns M (2009) Identification of 18 flutter derivatives by forced vibration tests: a new experimental rig. In Proceedings of the 5th European and African Conference on Wind Engineering (EACWE2009), Florence, Italy, paper no. 126.

Nieto F, Hernández S and Jurado JA (2008) Structural optimization of the flutter problem in suspension bridges: an analytical formulation. In Proceedings of the 6th International Colloquium on Bluff Body Aerodynamics and Applications (BBAA6), Milan, Italy, paper no. BDG 22.

Nieto F, Hernández S, Jurado JA and Mosquera A (2011) Analytical approach to sensitivity analysis of flutter speed in bridges considering variable deck mass. Advances in Engineering Software 42(4): 117-129.

Nieto F, Hargreaves D, Owen J and Hernández S (2015a) On the applicability of 2D URANS and SST $k-\omega$ turbulence model to the fluid-structure interaction of rectangular cylinders. Engineering Applications of Computational Fluid Mechanics 9(1) 157-173.

Nieto F, Owen J, Hargreaves D and Hernández S (2015b) Bridge deck flutter derivatives: efficient numerical evaluation exploiting their interdependence. Journal of Wind Engineering and Industrial Aerodynamics 136: 138-150, https://doi.org/10.1016/j.jweia.2014. 11.006.

Nikitas N, Jakobsen JB and Macdonald JHG (2008) Full-scale identification of modal and aeroelastic parameters of the Clifton suspension bridge. In Proceedings of the 6th International Colloquium on Bluff Body Aerodynamics and Applications (BBAA6), Milan, Italy, paper no. BDG12.

Nowicki T and Flaga A (2011) Modelling aeroelastic response of bridge decks using discrete vortex method. In Proceedings of the 19th International Conference on Computer Methods in Mechanics (CMM2011), Warsaw, Poland, paper no. 192.

Ogawa K, Shimodoi H and Oryu T (2002) Aerodynamic characteristics of a 2-box girder section adaptable for a super-long span suspension bridge. Journal of Wind Engineering and Industrial Aerodynamics 90(12-15): 2033-2043.

Øiseth O and Sigbjörnsson R (2011) An alternative analytical approach to prediction of flutter stability limits of cable supported bridges. Journal of Sound and Vibration 330(12) 2784-2800.

Øiseth O, Rönnquist A and Sigbjörnsson R (2010) Simplified prediction of wind-induced response and stability limit of slender long-span 
suspension bridges, based on modified quasi-steady theory: a case study. Journal of Wind Engineering and Industrial Aerodynamics 98(12): 730-741.

Øiseth O, Rönnquist A and Sigbjörnsson R (2011) Time domain modeling of self-excited aerodynamic forces for cable-supported bridges: a comparative study. Computers and Structures 89(13-14) 1306-1322.

Øiseth O, Rönnquist A and Sigbjörnsson R (2012) Finite element formulation of the self-excited forces for time-domain assessment of wind-induced dynamic response and flutter stability limit of cable-supported bridges. Finite Elements in Analysis and Design 50: 173-183, https://doi.org/10.1016/j.finel. 2011.09.008

Øiseth O, Rönnquist A and Sigbjörnsson R (2013) Prediction of wind-induced dynamic response and flutter stability limit of long-span bridges using the finite element method. In Proceedings of the 6th International Symposium on Strait Crossings 2013, pp. 164-174.

Ostenfeld C, Frandsen AG, Jessen JJ and Hass G (1970) Motorway Bridge Across Lillebcelt, Publication III: Design and Construction of the Bridge. Chr. Ostenfeld and W. Jonson, Copenhagen, Denmark, Report.

Ostenfeld-Rosenthar P, Madsen H and Larsen A (1992) Probabilistic flutter criteria for long span bridges. Journal of Wind Engineering and Industrial Aerodynamics 41-44(1-3): 1265-1276.

Park J, Morgenthal G, Kim K, Kwon SD and Law KH (2014) Power evaluation of flutter-based electromagnetic energy harvesters using computational fluid dynamics simulations. Journal of Intelligent Material Systems and Structures 25(14): 1-13.

Parkinson GV (1989) Phenomena and modeling of flow-induced vibrations of bluff bodies. Progress in Aerospace Sciences 26(2): 169-224.

Patruno L (2015) Accuracy of numerically evaluated flutter derivatives of bridge deck sections using rans: effects on the flutter onset velocity. Engineering Structures 89: 49-65, https://doi.org/10.1016/ j.engstruct.2015.01.034.

Peng J and Chen GS (2012) Flow-oscillating structure interactions and the applications to propulsion and energy harvest. Applied Physics Research 4(2): 1-14

Permata R, Yonamine K, Hattori H and Shirato H (2013) Aerodynamics and flutter stability of slender bridge deck with double slot and porous cavity. In Proceedings of the 6th Civil Engineering Conference in Asia Region (CECAR6), Jakarta, Indonesia, paper no. 215 , pp. $13-20$.

Peters D (2008) Two-dimensional incompressible unsteady airfoil theory - an overview. Journal of Fluids and Structures 24(3): 295-312.

Petrini F, Giuliano F and Bontempi F (2007) Comparison of time domain techniques for the evaluation of the response and the stability in long span suspension bridges. Computers and Structures 85(11): 1032-1048.

Phan DH and Nguyen NT (2013) Flutter and buffeting control of long-span suspension bridge by passive flaps: experiment and numerical simulation. Journal of Aeronautical and Space Sciences 14(1): 46-57.

Phongkumsing S, Wilde K and Fujino Y (2001) Analytical study on flutter suppression by eccentric mass method on FEM model of long-span suspension bridge. Journal of Wind Engineering and Industrial Aerodynamics 89(6): 515-534

Piña RB and Caracoglia L (2009) Extraction of flutter derivatives from small-scale wind tunnel experiments. In Proceedings of the 11th American Conference on Wind Engineering (ACWE11), San Jaun, Puerto Rico.
Pleif MS and Batista RC (1995) Aerodynamic stability analysis of cable-stayed bridges. Journal of Structural Engineering 121(12): $1784-1788$

Pourzeyanali S and Datta TK (2002) Reliability analysis of suspension bridges against flutter. Journal of Sound and Vibration 254(1): 143-162.

Put TVD (1976) Rigidity of structures against aerodynamic forces. In IABSE Congress Report. International Association for Bridge and Structural Engineering (IABSE), ETH Zurich, Zurich, Switzerland, vol. 36, pp. 189-196.

Qin XR and Gu M (2004) Determination of flutter derivatives by stochastic subspace identification technique. Wind and Structures An International Journal 7(3): 173-186.

Riley ME (2011) Quantification of Model-Form, Predictive, and Parametric Uncertainties in Simulation-Based Design. PhD thesis, Wright State University, Dayton, OH, USA

Riley ME and Grandhi RV (2010) Quantification of modeling uncertainty in aeroelastic design. In Proceedings of the 13th AIAAIISSMO Multidisciplinary Analysis Optimization Conference, Fort Worth, TX, USA. American Institute of Aeronautics and Astronautics, Reston, VA, USA.

Riley ME and Grandhi RV (2011) A method for the quantification of model-form and parametric uncertainties in physics-based simulations. In Proceedings of the 52nd AIAA/ASME/ASCE/ AHS/ASC Structures, Structural Dynamics and Materials Conference, Denver, CO, USA. American Institute of Aeronautics and Astronautics, Reston, VA, USA

Riley ME, Grandhi RV and Kolonay R (2010) Quantification of modeling uncertainty in aeroelastic design. In Proceedings of the 51 st AIAA/ASME/ASCE/AHS/ASC Structures, Structural Dynamics, and Materials Conference, Orlando, FL. American Institute of Aeronautics and Astronautics, Reston, VA, USA.

Robertson I, Sherwin S and Bearman P (2001) Prediction of flutter instability due to cross winds of the second Forth Road Bridge. In Proceedings of the European Congress on Computational Methods in Applied Sciences and Engineering (ECCOMAS), Swansea, Wales.

Roger KL (1977) Airplane math modeling methods for active control design. In Structural Aspects of Active Control, AGARD-CP-228, pp. 4.1-4.11.

Salvatori L and Borri C (2007) Frequency- and time-domain methods for numerical modeling of full-bridge aeroelasticity. Computers and Structures 85(11-14): 657-687.

Salvatori L and Spinelli P (2007) A discrete 3D model for bridge aerodynamics and aeroelasticity: nonlinearities and linearizations. Meccanica 42(1): 31-46.

Sarkar P, Caracoglia L and Haan F (2007) Parametric study of flutter derivatives of bluff cross sections and their implications on the aeroelastic stability of flexible bridges. Proceedings of the 39th Joint Meeting of US/Japan Natural Resources Development Program (UJNR), Tsukuba, Japan, pp. 432-441.

Sarkar PP, Caracoglia L, Haan FL Jr, Sato H and Murakoshi J (2009) Comparative and sensitivity study of flutter derivatives of selected bridge deck sections, part 1: analysis of inter-laboratory experimental data. Engineering Structures 31(1): 158-169.

Šarkić A (2014) Validated Numerical Simulation of Fluid-Structure Interactions of Bridge Girders in Turbulent Wind Fields. PhD thesis, Ruhr-Universität Bochum, Bochum, Germany.

Šarkić A, Fisch R, Bletzinger KU and Höffer R (2011) Bridge flutter derivatives based on computed, validated pressure fields. In Proceedings of the 13th International Conference on Wind Engineering (ICWE13), Amsterdam, the Netherlands. 
Šarkić A, Fisch R, Höffer R and Bletzinger KU (2012) Bridge flutter derivatives based on computed, validated pressure fields. Journal of Wind Engineering and Industrial Aerodynamics 104-106: 141-151, https://doi.org/10.1016/j.jweia.2012.02.033.

Šarkić A, Höffer R and Brčić S (2015) Numerical simulations and experimental validations of force coefficients and flutter derivatives of a bridge deck. Journal of Wind Engineering and Industrial Aerodynamics 144: 172-182, https://doi.org/10.1016/j.jweia.2015. 04.017.

Sarwar M, Ishihara T, Shimada K, Yamasaki Y and Ikeda T (2008) Prediction of aerodynamic characteristics of a box girder bridge section using the LES turbulence model. Journal of Wind Engineering and Industrial Aerodynamics 96(10-11): 1895-1911.

Sato H, Hirahara N, Fumoto K, Hirano S and Kusuhara S (2002) Full aeroelastic model test of a super long-span bridge with slotted box girder. Journal of Wind Engineering and Industrial Aerodynamics 90(12-15): 2023-2032.

Scanlan RH (1978a) The action of flexible bridges under wind, I: flutter theory. Journal of Sound and Vibration 60(2): 187-199.

Scanlan RH (1978b) The action of flexible bridges under wind, II: buffeting theory. Journal of Sound and Vibration 60(2): 201-211.

Scanlan RH (1984) Role of indicial functions in buffeting analysis of bridges. Journal of Structural Engineering 110(7): 1433-1446.

Scanlan R (1987) Interpreting aeroelastic models of cable-stayed bridges. Journal of Engineering Mechanics 113(4): 555-575.

Scanlan RH (1993) Problematics in formulation of wind-force models for bridge decks. Journal of Engineering Mechanics 119(7): 1353-1375.

Scanlan RH (1997a) Amplitude and turbulence effects on bridge flutter derivatives. Journal of Structural Engineering 123(2): 232-236.

Scanlan RH (1997b) Some observations on the state of bluff-body aeroelasticity. Journal of Wind Engineering and Industrial Aerodynamics 69-71: 77-90, https://doi.org/10.1016/S0167-6105 (97)00148-7.

Scanlan RH (2000) Motion-related body-force functions in two-dimensional low-speed flow. Journal of Fluids and Structures 14(1): 49-63.

Scanlan RH (2001) Reexamination of sectional aerodynamic force functions for bridges. Journal of Wind Engineering and Industrial Aerodynamics 89(14-15): 1257-1266.

Scanlan RH (2002) Observations on low-speed aeroelasticity. $A S C E$ Journal of Engineering Mechanics 128(12): 1254-1258.

Scanlan RH and Jones NP (1990) Aeroelastic analysis of cable-stayed bridges. Journal of Structural Engineering 116(2): 279-297.

Scanlan RH and Tomko JJ (1971) Airfoil and bridge deck flutter derivatives. ASCE Journal of Engineering Mechanics 97(6): $1717-1737$.

Scanlan RH, Beliveau JG and Budlong KS (1974) Indicial aerodynamic functions for bridge decks. Journal of Engineering Mechanics 100(4): 657-672.

Scanlan R, Jones N and Singh L (1997) Inter-relations among flutter derivatives. Journal of Wind Engineering and Industrial Aerodynamics 69-71: 829-837, https://doi.org/10.1016/S0167-6105 (97)00209-2.

Selberg A (1961) Oscillation and aerodynamic stability of suspension bridges. In Acta Polytechnica Scandinavica: Civil Engineering and Building Construction, 13.

Selvam RP and Govindaswamy S (2001) Aeroelastic Analysis of Bridge Girder Section using Computer Modelling. Mack Blackwell Transportation Center, University of Arkansas, Fayetteville, AR, USA, Report.
Seo DW (2013) Effects of Errors in Flutter Derivatives on the WindInduced Response of Cable-Supported Bridges. $\mathrm{PhD}$ thesis, Northeastern University, Boston, MA, USA.

Seo DW and Caracoglia L (2011a) Estimation of torsional-flutter probability in flexible bridges considering randomness in flutter derivatives. Engineering Structures 33(8): 2284-2296.

Seo DW and Caracoglia L (2011b) Monte-Carlo methods for estimating the buffeting response of a bridge contaminated by flutter-derivative errors. In Proceedings of the 13th International Conference on Wind Engineering (ICWE13), Amsterdam, the Netherlands, paper no. 241.

Seo D and Caracoglia L (2013) Lifetime cost analysis of slender bridges due to flutter occurrence using the data of the United States Japan benchmark study on flutter derivatives. In Proceedings of the 44th Joint Meeting of US-Japan Panel on Wind and Seismic Effects (UJNR), Gaithersburg, MD, USA.

Shirai S and Ueda T (2003) Aerodynamic simulation by CFD on flat box girder of super-long-span suspension bridge. Journal of Wind Engineering and Industrial Aerodynamics 91(1): 279-290.

Shufang S, Zhenzhou L, Weiwei Z and Zhengyin Y (2009) Reliability and sensitivity analysis of transonic flutter using improved line sampling technique. Chinese Journal of Aeronautics 22(5): 513-519.

Simiu E and Scanlan RH (1996) Wind Effects on Structures: Fundamentals and Applications to Design, 3rd edn. J. Wiley and Sons, New York, NY, USA.

Singh K, Michelin S and de Langre E (2012) The effect of non-uniform damping on flutter in axial flow and energy-harvesting strategies. Proceedings of the Royal Society A 468(2147): 3620-3635.

Stærdahl JW, Sørensen N and Nielsen SRK (2007) Aeroelastic stability of suspension bridges using CFD. In Proceedings of the Symposium of the International Association for Shell and Spatial Structures (IASS), Venice, Italy.

Starossek U (1992) Brückendynamik: Winderregte Schwingungen von Seilbrücken. Friedr. Vieweg \& Sohn Verlagsgesellschaft GmbH, Braunschweig/Wiesbaden, Germany (in German).

Starossek U (1993) Prediction of flutter through the use of finite elements. Structural Engineering Review 5(4): 301-307.

Starossek U (1994) Simplified flutter prediction for bridges with bluff cross-section. Structural Engineering Review 6(1): 35-38.

Starossek U (1997) Bridge flutter prediction with finite beam element in complex notation. Proceedings of the 7th International Conference on Computing in Civil and Building Engineering (ICCCBE-VII), Seoul, South Korea, pp. 2099-2104.

Starossek U (1998a) Bridge instability in wind and spatial flutter analysis. In Proceedings of the Korean Society of Civil Engineers Annual Conference, Seoul, Korea.

Starossek U (1998b) Complex notation in flutter analysis. Journal of Structural Engineering, ASCE 124(8): 975-977.

Starossek U, Aslan H and Thiesemann L (2009) Experimental and numerical identification of flutter derivatives for nine bridge deck sections. Wind and Structures 12(6): 519-540.

Strømmen E (2006) Theory of Bridge Aerodynamics. Springer, Berlin/Heidelberg, Germany.

Sun D, Owen JS and Wright NG (2009) Application of the $k$-omega turbulence model for a wind-induced vibration study of 2D bluff bodies. Journal of Wind Engineering and Industrial Aerodynamics 97(2): 77-87.

Sun D, Owen JS, Wright NG and Liaw KF (2008) Fluid-structure interaction of prismatic line-like structures, using LES and block-iterative coupling. Journal of Wind Engineering and Industrial Aerodynamics 96(6-7): 840-858. 
Svensson H (2013) Cable-Stayed Bridges: 40 Years of Experience Worldwide. Wilhelm Ernst \& Sohn, Berlin, Germany.

Szabó G and Györgyi J (2009a) Fluid-structure interaction analysis with the ANSYS software in bridge aeroelasticity. In Proceedings of the 5th European and African Conference on Wind Engineering (EACWE2009), Florence, Italy, paper no. 87.

Szabó G and Györgyi J (2009b) Three-dimensional fluid-structure interaction analysis for bridge aeroelasticity. In Proceedings of the World Congress on Engineering and Computer Science (WCECS2009), San Francisco, USA.

Szabó G and Györgyi J (2011) Numerical simulation of the flutter performance of different generic bridge cross sections. Journal of Civil Engineering 55(2): 137-146.

Szechenyi E (1973) Etude du comportement aéroélastique du pont de Saint-Nazaire à Saint-Brévin. ONERA, Palaiseau, France, Report NT 2/3044 RY (in French).

Tamura Y and Kareem A (2013) Advanced Structural Wind Engineering. Springer, Tokyo, Japan.

Tanaka H, Yamamura N and Tatsumi M (1992) Coupled mode flutter analysis using flutter derivatives. Journal of Wind Engineering and Industrial Aerodynamics 41-44(1-3): 1279-1290.

Tang D, Yamamoto $\mathrm{H}$ and Dowell EH (2003) Flutter and limit cycle oscillations of two-dimensional panels in three-dimensional axial flow. Journal of Fluids and Structures 17(2): 225-242.

Taylor I and Vezza M (1999) Prediction of unsteady flow around square and rectangular section cylinders using a discrete vortex method. Journal of Wind Engineering and Industrial Aerodynamics 82(1-3): $247-269$.

Thang ND, Katsuchi H, Yamada H and Saski E (2008) Effects of approximation of self-excited forces by rational function on wind-induced response of long span bridge. Journal of Structural Engineering 54: 420-428, http://doi.org/10.11532/structcivil. 54A.420.

Theodorsen T (1935) General Theory of Aerodynamic Instability and the Mechanism of Flutter. National Advisory Committee for Aeronautics (NACA), Langley Field, VA, USA, Report 496.

Theodorsen T and Garrick IE (1940) Mechanism of Flutter A Theoretical and Experimental Investigation of the Flutter Problem. NACA, Langley Field, VA, USA, Report 685.

Thiesemann L and Starossek U (2003) Numerische und experimentelle ermittlung von instationären luftkräften zum nachweis der aeroelastischen stabilität weitgespannter brücken. In Forschungskolloquium Baustatik-Baupraxis, Neubiberg, Munich, Germany, pp. 247-258 (in German).

Thiesemann L, Bergmann D and Starossek U (2003) Numerical and experimental evaluation of flutter derivatives by means of the forced vibration method. In Proceedings of the 11th International Conference on Wind Engineering (ICWE2003), Lubbock, TX, USA, vol. 2, pp. 1571-1578.

Tiffany SH and Adams WM Jr (1984) Fitting Aerodynamic Forces in the Laplace Domain: An Application of Nonlinear Nongradient Technique to Multilevel Constrained Optimization. National Aeronautics and Space Administration (NASA), Washington, DC, USA, Report TM-86317.

Tiffany SH and Adams WM Jr (1988) Nonlinear Programming Extensions to Rational Function Approximation Methods for Unsteady Aerodynamic Forces. National Aeronautics and Space Administration (NASA), Washington, DC, USA, Report NASA-TP-2776.

Trein CA and Shirato H (2011) Coupled flutter stability from the unsteady pressure characteristics point of view.
Journal of Wind Engineering and Industrial Aerodynamics 99(2): 114-122.

Tubino F (2005) Relationships among aerodynamic admittance functions, flutter derivatives and static coefficients for long-span bridges. Journal of Wind Engineering and Industrial Aerodynamics 93(12): 929-950.

Turbelin G and Gibert JR (1996) CFD calculations of indicial lift response for bluff bodies. Wind and Structures 5(2-4): 245-256.

University Libraries (2017) University Libraries - University of Washington, Washington, DC, USA. See http://www.lib. washington.edu (accessed 15/05/2017).

Unjoh S and Adachi Y (1998) Damping characteristics of long-span suspension bridges. In IABSE Congress Report. International Association for Bridge and Structural Engineering (IABSE), ITH-Zurich, Zurich, Switzerland.

Vairo G (2003) A numerical model for wind loads simulation on long-span bridges. Simulation Modelling Practice and Theory 11(5-6): 315-351.

Vairo G (2010) A simple analytical approach to the aeroelastic stability problem of long-span cable-stayed bridges. International Journal for Computational Methods in Engineering Science and Mechanics 11(1): $1-19$.

Vu TV, Lee HY, Chun NH et al. (2011) Flutter analysis of bridges through use of state space method. In Proceedings of the 8th International Conference on Structural Dynamics (EURODYN2011), Leuven, Belgium, pp. 3083-3090.

Vu TV, Kim YM and Lee HE (2015) Coupled flutter analysis of long-span bridges using full set of flutter derivatives. KSCE Journal of Civil Engineering 20(4): 1501-1513.

Wang Q and Liao H (2012) The nonlinear aerodynamic stability of longspan bridges: post flutter. In Proceedings of the 7th International Colloquium on Bluff Body Aerodynamics and Applications (BBAA7), Shanghai, China (Xiang H, Ge Y and Cao S (eds)). China Communications Press, Beijing, China, pp. 1371-1380.

Wagner H (1925) Über die entstehung des dynamischen auftriebes von tragflügeln. ZAMM - Journal of Applied Mathematics and Mechanics (Zeitschrift für Angewandte Mathematik und Mechanik) 5(1): 17-35 (in German).

Walther JH (1994) Discrete Vortex Method for Two-Dimensional Flow past Bodies of Arbitrary Shape Undergoing Prescribed Rotary and Translational Motion. PhD thesis, Technical University of Denmark (DTU), Kongens Lyngby, Denmark.

Walther JH and Larsen A (1997) Two dimensional discrete vortex method for application to bluff body aerodynamics. Journal of Wind Engineering and Industrial Aerodynamics 67-68: 183-193, https://doi.org/10.1016/S0167-6105(97)00072-X.

Washizu K, Ohya A, Otsuki Y and Fujii K (1978) Aeroelastic instability of rectangular cylinders in a heaving mode. Journal of Sound and Vibration 59(2): 195-210.

Washizu K, Ohya A, Otsuki Y and Fujii K (1980) Aeroelastic instability of rectangular cylinders in a torsional mode due to a transverse wind. Journal of Sound and Vibration 72(4): 507-521.

Watanabe S and Fumoto K (2008) Aerodynamic study of slotted box girder using computational fluid dynamics. Journal of Wind Engineering and Industrial Aerodynamics 96(10-11): 1885-1894.

Weight AJ (2009) Critical analysis of the Great Belt East Bridge, Denmark. In Proceedings of Bridge Engineering 2nd Conference, Bath, UK.

Wenzel H (2008) Health Monitoring of Bridges. Wiley, Chichester, UK. Wilde K, Fujino Y and Masukawa J (1996) Time domain modeling of bridge deck flutter. Journal of Structural Mechanics and Earthquake Engineering 13(2): 19-29. 
Wu JC, Liu WC and Juang JY (2006) Identification of flutter derivatives by forced vibration technique. In Proceedings of the 4th International Symposium on Computational Wind Engineering (CWE2006), Yokohama, Japan, pp. 785-788.

Wu T and Kareem A (2011) Modeling hysteretic nonlinear behavior of bridge aerodynamics via cellular automata nested neural network. Journal of Wing Engineering and Industrial Aerodynamics 99(4): 378-388.

Wu T and Kareem A (2013) Bridge aerodynamics and aeroelasticity: a comparison of modeling schemes. Journal of Fluids and Structures 43: 347-370, https://doi.org/10.1016/j.jfluidstructs.2013. 09.015 .

Wu T and Kareem A (2014a) A nonlinear convolution scheme to simulate bridge aerodynamics. Computers and Structures 128 : 259-271, https://doi.org/10.1016/j.compstruc.2013.06.004.

Wu T and Kareem A (2014b) Revisiting convolution scheme in bridge aerodynamics: comparison of step and impulse functions. Journal of Engineering Mechanics 140(5): 1-13.

Wu T and Kareem A (2014c) Simulation of nonlinear bridge aerodynamics: a sparse third-order Volterra model. Journal of Sound and Vibration 333(1): 178-188.

Wu T and Kareem A (2015) A nonlinear analysis framework for bluff-body aerodynamics: a Volterra representation of the solution of Navier-Stokes equations. Journal of Fluids and Structures 54: 479-502, https://doi.org/10.1016/j.jfluidstructs.2014.12.005.

Xiang $\mathrm{H}$ and $\mathrm{Ge} \mathrm{Y}$ (2002) Refinements on aerodynamic stability analysis of super long-span bridges. Journal of Wind Engineering and Industrial Aerodynamics 90(12-15): 1493-1515.

Xiang H, Liu C and Gu M (1995) Time-domain analysis for coupled buffeting response on long span bridge. Proceedings of the 9th International Conference on Wind Engineering (ICWE1995), New Delhi, India, pp. 881-892.

Xin D and Ou J (2007) Numerical simulation of aerodynamic derivatives and critical wind speed for long-span bridges based on simplified steady wind field. Earthquake Engineering and Engineering Vibration 6(2): 197-203.

Xin D, Ou J and Li H (2010) Flutter stability analysis of long-span bridge based on numerical calculation applying dynamic mesh technique. In Proceedings of the 4th International Symposium on Computational Wind Engineering (CWE2010), Chapel Hill, NC, USA, paper no. 196.

Xu FY (2014) Practical diagrammatical technique for 3-DOF bridge flutter analysis. Journal of Bridge Engineering 19(12): $1-12$.

Xu FY, Chen XZ, Cai CS and Chen AR (2012) Determination of 18 flutter derivatives of bridge decks by an improved stochastic search algorithm. Journal of Bridge Engineering 17(4): 576-588.

Xu F, Zhu L, Ge X and Zhang Z (2014) Some new insights into the identification of bridge deck flutter derivatives. Engineering Structures 75: 418-428, https://doi.org/10.1016/j.engstruct.2014. 06.015.

Xu X (2009a) Parametric studies on relationships between flutter derivatives of slender bridge (I). Applied Mathematics and Mechanics 30(2): 237-245.

Xu X (2009b) Parametric studies on relationships between flutter derivatives of slender bridge (II). Applied Mathematics and Mechanics 30(3): 335-341.

Xu YL (2013) Wind Effects on Cable-Supported Bridges. John Wiley \& Sons Singapore Pte. Ltd., Singapore, Singapore.

Yamada H, Katsuchi H and Kien PH (2006) A study on understanding of coupled flutter of long-span bridges. In Proceedings of the 4th International Symposium on Computational Wind Engineering (CWE2006), Yokohama, Japan, pp. 141-144.
Yang YX, Ge YJ and Xiang HF (2006) Flutter mechanism and flutter modality investigation for thin plate sections. In Proceedings of the 4th International Symposium on Computational Wind Engineering (CWE2006), Yokohama, Japan, pp. 501-504.

Yang Y, Ge Y and Xiang H (2007) Flutter control effect and mechanism of central-slotting for long-span bridges. Frontiers of Architecture and Civil Engineering in China 1(3): 298-304.

Yang YX, Ge YJ and Zhang W (2009) Investigation on the flutter mechanism of thin plate sections. In Proceedings of the 5 th European and African Conference on Wind Engineering (EACWE2009), Florence, Italy, paper no. 119.

Yang Y, Zhou R, Ge Y, Mohotti D and Mendis P (2015) Aerodynamic instability performance of twin box girders for long-span bridges. Journal of Wind Engineering and Industrial Aerodynamics 145: 196-208, https://doi.org/10.1016/j.jweia.2015.06.014.

Yoshimura T and Nakamura Y (1979) On the indicial aerodynamic aerodynamic moment responses of bridge deck sections. In Proceedings of the 5th International Conference on Wind Engineering, Fort Collins, CO, USA. Cambridge University Press, Cambridge, UK, pp. 877-855.

Yoshizumi F and Inoue H (2002) An experimental approach on aerodynamic stability of a cable-stayed cantilever bridge. Journal of Wind Engineering and Industrial Aerodynamics 90(12-15): 2099-2111.

Zasso A (1996) Flutter derivatives: advantages of a new representation convention. Journal of Wind Engineering and Industrial Aerodynamics 60: 35-47, https://doi.org/10.1016/01676105(96)00022-0.

Zhan H and Fang T (2012) Flutter stability studies of Great Belt East Bridge and Tacoma Narrows Bridge by CFD numerical simulation. In Proceedings of the 7th International Colloquium on Bluff Body Aerodynamics and Applications (BBAA7), Shanghai, China, (Xiang H, Ge Y and Cao S (eds)). China Communications Press, Beijing, China, pp. 1518-1527.

Zhan H, Fang T and Zhang Z (2013) Flutter stability studies of Great Belt East suspension bridge by two CFD numerical simulation methods. In Proceedings of the 6th European and African Conference on Wind Engineering (EACWE2013), Cambridge, UK.

Zhang X (2004) Investigation on aerodynamic stability of long-span suspension bridges under erection. Journal of Wind Engineering and Industrial Aerodynamics 92(1): 1-8.

Zhang X and Sun B (2004) Short note: parametric study on the aerodynamic stability of a long-span suspension bridge. Journal of Wind Engineering and Industrial Aerodynamics 92(6): 431-439.

Zhang X and Sun B (2005) Aerodynamic stability of cable-stayed-suspension hybrid bridges. Journal of Zhejiang University 6(8): 869-874.

Zhang X, Xiang H and Sun B (2002) Nonlinear aerostatic and aerodynamic analysis of long-span suspension bridges considering wind-structure interactions. Journal of Wind Engineering and Industrial Aerodynamics 90(9): 1065-1080.

Zhang X, Brownjohn JMW and Omenzetter P (2003a) Time domain formulation of self-excited forces on bridge deck for wind tunnel experiment. Journal of Wind Engineering and Industrial Aerodynamics 91(6): 723-736.

Zhang X, Sun B and Peng W (2003b) Study on flutter characteristics of cable-supported bridges. Journal of Wind Engineering and Industrial Aerodynamics 91(6): 841-854.

Zhang ZT and Chen ZQ (2010) Indicial functions for bridge aero-elastic forces and discussion of some problematic issues in flutter analysis. In Proceedings of the 4th International Symposium on Computational Wind Engineering (CWE2010), Chapel Hill, NC, USA, paper no. 408. 
Zhang Z, Chen Z, Cai Y and Ge Y (2011) Indicial functions for bridge aeroelastic forces and time-domain flutter analysis. Journal of Bridge Engineering 16(4): 546-557.

Zhou Z, Chen A and Xiang H (2003) Identification of aeroelastic parameter of flexible bridge decks by random discrete vortex method. In Proceedings of the 11th International Conference on Wind Engineering (ICWE11), Lubbock, TX, USA.

Zhou Z, Chen A and Xiang H (2006) On the mechanism of torsional flutter instability for 1st Tacoma narrow bridge by discrete vortex method. In Proceedings of the 4th International Symposium on Computational Wind Engineering (CWE2006), Yokohama, Japan, pp. 505-508.
Zhu LD, Xu YL and Xiang HF (2002) Tsing Ma bridge deck under skew winds - part II: flutter derivatives. Journal of Wind Engineering and Industrial Aerodynamics 90(7): 807-837.

Zhu LD, Chang GZ and Li C (2006) Skew wind effect on 2-DOF coupled flutter of a flat-box deck. In Proceedings of the 4th International Symposium on Computational Wind Engineering (CWE2006), Yokohama, Japan, pp. 497-500.

Zhu ZW, Gu M and Chen ZQ (2007) Wind tunnel and CFD study on identification of flutter derivatives of a long-span self-anchored suspension bridge. Computer-Aided Civil and Infrastructure Engineering 22(8): 541-554.

\section{How can you contribute?}

To discuss this paper, please email up to 500 words to the editor at journals@ice.org.uk. Your contribution will be forwarded to the author(s) for a reply and, if considered appropriate by the editorial board, it will be published as discussion in a future issue of the journal.

Proceedings journals rely entirely on contributions from the civil engineering profession (and allied disciplines). Information about how to submit your paper online is available at www.icevirtuallibrary.com/page/authors, where you will also find detailed author guidelines. 